\title{
CONTACT METRIC THREE MANIFOLDS AND LORENTZIAN GEOMETRY WITH TORSION IN SIX-DIMENSIONAL SUPERGRAVITY
}

\author{
ÁNGEL MURCIA AND C. S. SHAHBAZI
}

\begin{abstract}
We introduce the notion of $\varepsilon \eta$-Einstein $\varepsilon$-contact metric three-manifold, which includes as particular cases $\eta$-Einstein Riemannian and Lorentzian (para) contact metric threemanifolds, but which in addition allows for the Reeb vector field to be null. We prove that the product of an $\varepsilon \eta$-Einstein Lorentzian $\varepsilon$-contact metric three-manifold with an $\varepsilon \eta$-Einstein Riemannian contact metric three-manifold carries a bi-parametric family of Ricci-flat Lorentzian metric-compatible connections with isotropic, totally skew-symmetric, closed and co-closed torsion, which in turn yields a bi-parametric family of solutions of six-dimensional minimal supergravity coupled to a tensor multiplet. This result allows for the systematic construction of families of Lorentzian solutions of six-dimensional supergravity from pairs of $\varepsilon \eta$-Einstein contact metric three-manifolds. We classify all left-invariant $\varepsilon \eta$-Einstein structures on simply connected Lie groups, paying special attention to the case in which the Reeb vector field is null. In particular, we show that the Sasaki and K-contact notions extend to $\varepsilon$-contact structures with null Reeb vector field but are however not equivalent conditions, in contrast to the situation occurring when the Reeb vector field is not light-like. Furthermore, we pose the Cauchy initial-value problem of an $\varepsilon$-contact $\varepsilon \eta$-Einstein structure, briefly studying the associated constraint equations in a particularly simple decoupling limit. Altogether, we use these results to obtain novel families of six-dimensional supergravity solutions, some of which can be interpreted as continuous deformations of the maximally supersymmetric solution on $\widetilde{\mathrm{Sl}}(2, \mathbb{R}) \times S^{3}$.
\end{abstract}

\section{Contents}

1. Introduction

2. $\varepsilon$-Contact metric three-manifolds 5

3. Null contact metric structures $\quad 12$

4. $\varepsilon \eta$-Einstein $\varepsilon$-contact metric manifolds 22

5. Six-dimensional supergravity and $\varepsilon$-contact structures 39

6. Ricci flat Lorentzian six-manifolds with closed self-dual torsion $\quad 41$

Appendix A. Simply connected three-dimensional Lorentzian Lie groups 43

Appendix B. Curvature of left-invariant metrics on Lorentzian Lie groups 46

$\begin{array}{ll}\text { References } & 48\end{array}$

Key words and phrases. Lorentzian geometry with torsion, contact metric manifolds, $\eta$-Einstein manifolds, supergravity.

2010 MSC. Primary: 53C50. Secondary: 53C25. 


\section{INTRODUCTION}

The ongoing mathematical study and development of supergravity [21, 30, 48] poses novel and occasionally striking mathematical problems in diverse areas of differential geometry and topology. Some of these mathematical problems are specifically concerned with the classification of supergravity solutions and the study of the associated moduli spaces of solutions. In this context, the classification of all simply connected Lorentzian manifolds admitting bosonic solutions of supergravity in the considered dimension remains as an outstanding open problem in the mathematical theory of supergravity. The purpose of this note is to propose a contribution to this problem in the specific case of minimal supergravity in six Lorentzian dimensions [46, 47] coupled to a tensor multiplet with constant dilaton, a theory that can be neatly rephrased as a natural geometric problem in the realm of six-dimensional Lorentzian geometry with torsion. More concretely, the bosonic configuration space $\operatorname{Conf}(M)$ of this six-dimensional supergravity (with constant dilaton) on a sixmanifold $M$ consists on pairs $(\mathrm{g}, \mathrm{H})$, where $\mathrm{g}$ is a Lorentzian metric on $M$ and $\mathrm{H}$ is a three-form. The bosonic theory is then geometrically defined through the following system of partial differential equations [46]:

$$
\operatorname{Ric}\left(\nabla^{\mathrm{H}}\right)=0, \quad \mathrm{dH}=0, \quad \mathrm{~d} *_{\mathrm{g}} \mathrm{H}=0, \quad|\mathrm{H}|_{\mathrm{g}}^{2}=0,
$$

for pairs $(\mathrm{g}, \mathrm{H}) \in \operatorname{Conf}(M)$, where $\nabla^{\mathrm{H}}$ is the unique metric-compatible (with respect to $g$ ) connection with totally skew-symmetric torsion $\mathrm{H}$. Therefore, solutions of minimal supergravity consist on six-dimensional Lorentzian manifolds equipped with a Ricci-flat metric-compatible connection with isotropic, skew-symmetric, closed and co-closed torsion. Recall that in six Lorentzian dimensions the Hodge operator on three-forms squares to the identity, and the metric induced by $g$ on the bundle of three-forms is of split signature. In particular, if $\mathrm{H}$ is closed and (anti) self-dual then it is automatically isotropic and co-closed, providing a natural candidate to solve Equations (1). (Anti) self-duality of $\mathrm{H}$ is in this context a clear reminiscent of the self-duality condition on the curvature of a connection on a principal U(1) bundle over a four-dimensional Riemannian manifold. This analogy can be made rigorous by using the theory of abelian gerbes [14], although we shall not pursue this point of view here. To the best of our knowledge the geometric problem posed by Equations (1) has not been studied in the mathematical literature.

A solution (g, H) of Equations (1) is said to be supersymmetric if there exists an irreducible real chiral spinor $\epsilon$ parallel with respect to the lift of $\nabla^{\mathrm{H}}$ to the spinor bundle (assuming the latter exists and is associated to a spin structure on $(M, \mathrm{~g})$ ) and sitting in the kernel of the endomorphism defined by $\mathrm{H}$ in the spinor bundle through Clifford multiplication. Supersymmetric solutions of six-dimensional supergravity have been extensively studied in the literature, see for instance $[1,2$, $7,20,22,25,28,34,35]$ and references therein. However, due to the complexity of the equations involved, examples of explicit solutions are scarce even in the restricted supersymmetric case, with some celebrated exceptions, see [7, 40-42] and their citations. Rather than studying the problem of classifying solutions to minimal supergravity coupled to a tensor multiplet in its full generality, which seems to be currently out of reach, in this article we make the following simplifying assumptions, with the aim of developing a method to construct families of solutions as a first step to understand the general classification problem:

- We assume that $M=N \times X$ is a direct product of three-dimensional oriented manifolds.

- We consider configurations $(\mathrm{g}, \mathrm{H}) \in \operatorname{Conf}(M)$ with a direct product metric $\mathrm{g}=\chi \oplus h$, where $\chi$ is a Lorentzian metric on $N$ and $h$ is a Riemannian metric on $X$.

With these provisos in mind, we obtain the following result, see Theorem 5.4 for more details. 
Theorem 1.1. Let $\left(N, \chi, \alpha_{N}, \varepsilon_{N}\right)$ be a Lorentzian $\varepsilon \eta$-Einstein $\varepsilon$-contact metric three-manifold and let $\left(X, h, \alpha_{X}\right)$ be a Riemannian $\varepsilon \eta$-Einstein contact metric three-manifold. Then, for appropriate choices of parameters in the $\varepsilon \eta$-Einstein condition (specified in Theorem 5.4), the oriented Cartesian product manifold

$$
M=N \times X,
$$

carries a family of solutions of six-dimensional minimal supergravity coupled to a tensor multiplet given by

$$
\mathrm{g}=\chi \oplus h, \quad \mathrm{H}_{\lambda, l}=\lambda \nu_{\chi}+\frac{l}{3}\left(*_{\chi} \alpha_{N}\right) \wedge \alpha_{X}+\frac{l}{3} \alpha_{N} \wedge\left(*_{h} \alpha_{X}\right)+\lambda \nu_{h}
$$

and parametrized by $(\lambda, l) \in \mathbb{R}^{2}$. Equivalently, $(M=N \times X, \mathrm{~g}=\chi \oplus h)$ admits a bi-parametric family of metric-compatible Ricci-flat connections $\nabla^{\mathrm{H}_{\lambda, l}}$ with totally skew-symmetric, isotropic, closed and co-closed torsion $\mathrm{H}_{\lambda, l}$.

Note that, as explained in Section 5, although the Levi-Civita connection $\nabla$ is a product connection, the torsionful connection $\nabla^{\mathrm{H}_{\lambda, l}}$ does not split in general. The notion of $\varepsilon$-contact structure is defined in Section 2, and contains as a particular case the standard notions of Riemannian contact structure when $g$ is Riemannian and the standard notion of Lorentzian (para) contact structure when $(\varepsilon=1)$ $\varepsilon=-1$. The novelty in the definition of $\varepsilon$-contact structure occurs in the case $\varepsilon=0$, namely the case in which the Reeb vector field is null. To the best of our knowledge this case has not been considered in the literature, and hence we study it in more detail. Consequently, we develop the notions of K-contact and Sasakian null contact metric structure, in analogy with the corresponding definitions occurring in the cases $\varepsilon \neq 0$. We show that the Sasakian condition is equivalent to the integrability of certain nilpotent endomorphism on $\mathbb{R} \times N$, which allows to characterize such structures similarly to the case $\varepsilon \neq 0$. On the other hand, we explicitly show that, in contrast to the three-dimensional $\varepsilon \neq 0$ case, the Sasakian and K-contact conditions are not equivalent if $\varepsilon=0$. Furthermore, we classify all left-invariant Lorentzian three-dimensional simply connected and connected Lie groups carrying left-invariant null contact structures, elucidating which of these structures are Sasakian or K-contact and finding the structure explicitly.

The notion of $\varepsilon \eta$-Einstein $\varepsilon$-contact structure is, for $\varepsilon \neq 0$, a restricted version of the standard $\eta$-Einstein condition considered in the literature of contact structures, see for example [9, 15], which is adapted to make Theorem 1.1 hold. In order to apply Theorem 1.1 to the construction of explicit solutions of minimal supergravity coupled to a tensor multiplet with constant dilaton, we classify all left-invariant $\varepsilon \eta$-Einstein $\varepsilon$-contact structures by exploiting the classification of simply connected three-dimensional Lorentzian Lie groups, developed in [24, 49]. We tabulate our results in the following form.

Theorem 1.2. A three-dimensional connected and simply connected Lie group $\mathrm{G}$ admits a leftinvariant $\varepsilon \eta$-Einstein contact structure $(g, \alpha)$ with time-like Reeb vector field if and only if $(\mathrm{G}, g, \alpha)$ is isomorphic, through a possibly orientation-reversing isometry, to one of the items listed in the following table in terms of the orthonormal frame $\left\{e_{0}, e_{1}, e_{2}\right\}$ appearing in Theorem A.1: 


\begin{tabular}{|c|c|c|c|c|c|}
\hline $\mathfrak{g}$ & Structure constants & $\alpha$ & $\eta$-Einstein constants & $\mathrm{G}$ & Sasakian \\
\hline \multirow{5}{*}{$\mathfrak{g}_{3}$} & $\frac{1}{2}>a=1-b>0, c=1$ & $\alpha=e^{0}$ & $\lambda^{2}=\kappa=2 b(1-b)$ & $\widetilde{\mathrm{Sl}}(2, \mathbb{R})$ & No \\
\cline { 2 - 7 } & $a=c=1, b=0$ & $\alpha=-e^{0}$ & $\lambda^{2}=\kappa=0$ & $\widetilde{\mathrm{E}}(1,1)$ & No \\
\cline { 2 - 7 } & $1 \geq a=b>0, c=1$ & $\alpha=e^{0}$ & $\lambda^{2}=1-\kappa=a$ & $\widetilde{\mathrm{Sl}}(2, \mathbb{R})$ & Yes \\
\cline { 2 - 7 } & $a=b=0, c=-1$ & $\alpha=-e^{0}$ & $\lambda^{2}=0, \kappa=1$ & $\mathrm{H}_{3}$ & Yes \\
\hline $\mathfrak{g}_{6}$ & $b=1, c=d=0,1 \geq a^{2}>0$ & $\alpha=e^{0}$ & $\lambda^{2}=1-\kappa=a^{2}$, & $\mathfrak{G}_{6}$ & Yes \\
\hline
\end{tabular}

Theorem 1.3. A three-dimensional connected and simply connected Lie group $G$ admits a leftinvariant $\varepsilon \eta$-Einstein para-contact structure $(g, \alpha)$ if and only if $(\mathrm{G}, g, \alpha)$ is isomorphic, through a possibly orientation-reversing isometry, to one of the items listed in the following table in terms of the orthonormal frame $\left\{e_{0}, e_{1}, e_{2}\right\}$ appearing in Theorem A.1:

\begin{tabular}{|c|c|c|c|c|c|}
\hline $\mathfrak{g}$ & Structure constants $\left(s \in \mathbb{Z}_{2}\right)$ & $\alpha$ & $\eta$-Einstein constants & $\mathrm{G}$ & Sasakian \\
\hline \multirow{5}{*}{$\mathfrak{g}_{3}$} & $a=s, b=c, s c \geq 1$ & $\alpha= \pm e^{1}$ & $\lambda^{2}=s c, \kappa=s c-1$ & $\widetilde{\mathrm{Sl}}(2, \mathbb{R})$ & Yes \\
\hline & $b=s, a=c, s c \geq 1$ & $\alpha= \pm e^{2}$ & $\lambda^{2}=s c, \kappa=s c-1$ & $\widetilde{\mathrm{Sl}}(2, \mathbb{R})$ & Yes \\
\hline & $b=0, a=c=s$ & $-\alpha_{0}^{2}+\alpha_{1}^{2}=1$ & $\lambda^{2}=0, \kappa=0$ & $\widetilde{\mathrm{E}}(1,1)$ & No \\
\hline & $c=0, a=b=s$ & $\alpha_{1}^{2}+\alpha_{2}^{2}=1$ & $\lambda^{2}=0, \kappa=0$ & $\widetilde{\mathrm{E}}(2)$ & No \\
\hline & $a=b=c=s$ & $-\alpha_{0}^{2}+\alpha_{1}^{2}+\alpha_{2}^{2}=1$ & $\lambda^{2}=1, \kappa=0$ & $\widetilde{\mathrm{Sl}}(2, \mathbb{R})$ & Yes \\
\hline \multirow[b]{2}{*}{$\mathfrak{g}_{6}$} & $a=b=0, d^{2} \geq 1, c=-s$ & $\alpha_{2}^{2}=1$ & $\lambda^{2}=d^{2}, \kappa=d^{2}-1$ & \multirow[b]{2}{*}{$\mathfrak{G}_{6}$} & Yes \\
\hline & $\begin{array}{c}b=-\mu a \neq 0, d=-a+\mu s \\
c=-s+\mu a, \mu \in \mathbb{Z}_{2}\end{array}$ & $\begin{array}{c}a \alpha_{2}=(-s+\mu a) \alpha_{0} \\
\alpha_{2}^{2}=1+\alpha_{0}^{2}\end{array}$ & $\lambda^{2}=1, \kappa=0$ & & Yes \\
\hline
\end{tabular}

Theorem 1.4. A three-dimensional connected and simply connected Lie group G admits a leftinvariant $\varepsilon \eta$-Einstein null contact structure $(g, \alpha)$ if and only if $(\mathrm{G}, g, \alpha)$ is isomorphic, through a possibly orientation-reversing isometry, to one of the items listed in the following table in terms of the orthonormal frame $\left\{e_{0}, e_{1}, e_{2}\right\}$ appearing in Theorem A.1:

\begin{tabular}{|c|c|c|c|c|c|}
\hline $\mathfrak{g}$ & Structure constants $\left(s \in \mathbb{Z}_{2}\right)$ & $\alpha$ & $\eta$-Einstein constants & $\mathrm{G}$ & Sasakian \\
\hline \multirow[b]{2}{*}{$\mathfrak{g}_{3}$} & $a=b=c=s$ & $\alpha_{0}^{2}=\alpha_{1}^{2}+\alpha_{2}^{2}$ & $\lambda^{2}=1, \kappa=0$ & $\widetilde{\mathrm{Sl}}(2, \mathbb{R})$ & Yes \\
\hline & $a=c=s, b=0$ & $\begin{array}{l}\alpha_{2}=0 \\
\alpha_{0}^{2}=\alpha_{1}^{2}\end{array}$ & $\lambda^{2}=0, \kappa=0$ & $\widetilde{\mathrm{E}}(1,1)$ & No \\
\hline \multirow{2}{*}{$\mathfrak{g}_{4}$} & $b=0, a=s$ & \multirow{2}{*}{$\begin{array}{c}\alpha_{1}=0, \\
\alpha_{0}=\mu \alpha_{2}\end{array}$} & $\lambda^{2}=1, \alpha_{0}^{2} \kappa=1$ & $\widetilde{\mathrm{Sl}}(2, \mathbb{R})$ & Yes \\
\hline & $b=0, a=0$ & & $\lambda^{2}=0, \alpha_{0}^{2} \kappa=2$ & $\widetilde{\mathrm{E}}(1,1)$ & No \\
\hline $\mathfrak{g}_{6}$ & $\begin{array}{c}a=d \neq 0, b=c \\
a=\mu(b+s), \mu \in \mathbb{Z}_{2}\end{array}$ & $\begin{array}{c}\alpha_{1}=0 \\
\alpha_{0}=-\mu \alpha_{2}\end{array}$ & $\begin{array}{c}\lambda^{2}=4 a^{2}, \\
\kappa=0\end{array}$ & $\mathfrak{G}_{6}$ & If $a=\frac{\mu s}{2}$ \\
\hline
\end{tabular}


Here $\widetilde{\mathrm{Sl}}(2, \mathbb{R})$ denotes the universal cover of $\mathrm{Sl}(2, \mathbb{R}), \widetilde{\mathrm{E}}(2)$ denotes the universal cover of the group of rigid motions of the Euclidean plane, whereas $\widetilde{E}(1,1)$ denotes the universal cover of the group of rigid motions of the Minkowski plane and $\mathrm{H}_{3}$ denotes the three-dimensional Heisenberg group. The classification of complete and simply connected $\varepsilon \eta$-Einstein Riemannian three-manifolds was completed in $[10,11,38]$. The classification of complete $\varepsilon \eta$-Einstein Lorentzian three-manifolds with time-like Reeb vector field, presented in Theorem 1.2, proceeds along similar lines to the Riemannian case and therefore may be known to experts, although we have not been able to find it explicitly stated in the literature. By appropriately combining $\varepsilon \eta$-Einstein Riemannian three manifolds with the $\varepsilon \eta$-Einstein $\varepsilon$-contact manifolds listed in the previous theorems, as prescribed in Section 5, we can obtain simply-connected six-dimensional Lorentzian manifolds equipped with a continuous family of Ricci-flat metric-compatible connections with totally skew symmetric, isotropic, closed and co-closed torsion. These and all their smooth discrete quotients carry solutions of minimal supergravity coupled to a tensor multiplet with constant dilaton.

The $\varepsilon \eta$-Einstein condition on a globally hyperbolic Lorentzian $\varepsilon$-contact manifold can be interpreted as a dynamical evolution problem for a metric, a one-form and a pair of functions on a Riemann surface. As such, it admits an initial value formulation. We develop such formulation in Section 4, with the goal of obtaining the associated constraint equations as a first step towards a future study of the Cauchy problem for these structures. This point of view and approach to Lorentzian $\varepsilon \eta$-Einstein structures seems to be largely missing in the mathematical literature, with some notable exceptions, see for instance [26].

The outline of this article is as follows. In Section 2 we introduce the notion of $\varepsilon$-contact structure and explore some of its generic properties, including its formulation on a globally hyperbolic Lorentzian three-manifold. Section 3 is devoted to $\varepsilon$-contact structures with light-like Reeb vector field, introducing the concepts of Sasakian and null K-contact contact structures and classifying those on a simply connected three-dimensional Lie group which are left-invariant. In Section 4 we introduce the notion of $\varepsilon \eta$-Einstein $\varepsilon$-contact structure and we classify all left-invariant $\varepsilon \eta$ Einstein $\varepsilon$-contact structures on simply connected three-dimensional Lie groups. In Section 5 we present Theorem 1.1 and we establish the link between $\varepsilon \eta$-Einstein $\varepsilon$-contact structures and solutions of supergravity in six dimensions. Finally, in Section 6 we illustrate the type of solutions of six-dimensional supergravity that are obtained through Theorem 1.1 by using the $\varepsilon \eta$-Einstein $\varepsilon$-contact manifolds obtained in Theorems $1.2,1.3$ and 1.4.

Acknowledgements. We thank Vicente Cortés, Tomás Ortín, Francisco Presas and Marco Zagermann for very useful discussions. Furthermore we thank an anonymous referee of JGP for several comments which have helped improving the manuscript. The work of C.S.S. is supported by the Humboldt foundation through the Humboldt grant ESP 1186058 HFST-P and the German Science Foundation (DFG) under the Research Training Group 1670 Mathematics inspired by String Theory. The work of AM is funded by the Spanish FPU Grant No. FPU17/04964. AM was further supported by the MCIU/AEI/FEDER UE grant PGC2018-095205-B-I00, and by the "Centro de Excelencia Severo Ochoa" Program grant SEV-2016-0597.

\section{2. $\varepsilon$-CONTACT METRIC THREE-MANIFOLDS}

In this section we introduce the notion of $\varepsilon$-contact metric structure, which encompasses as particular cases the standard definition of contact Riemannian metric structure, contact Lorentzian structure and para-contact metric structure in three dimensions, but which also allows for the Reeb vector field to be null. 
Definition 2.1. Let $M$ be an oriented three-manifold. An $\varepsilon$-contact metric structure (or $\varepsilon$ contact structure, in short) on $M$ consists of a triple $(g, \alpha, \varepsilon)$, with $\varepsilon \in\{-1,0,1\}, g$ a Riemmanian or pseudo-Riemannian metric on $M$ and $\alpha$ a one-form $\alpha \in \Omega^{1}(M)$, satisfying:

$$
\alpha=* \mathrm{~d} \alpha, \quad|\alpha|_{g}^{2}=\varepsilon,
$$

where $*: \Omega^{r}(M) \rightarrow \Omega^{3-r}(M)(r=0,1,2,3)$ denotes the Hodge-dual with respect to $g$ and the fixed orientation on $M$, which is then said to be an $\varepsilon$-contact metric three-manifold (or $\varepsilon$-contact threemanifold, in short). When $g$ is Lorentzian, we will assume that $(M, g)$ is oriented and time-oriented.

Remark 2.2. Note that equation $\alpha=* \mathrm{~d} \alpha$ is equivalent to

$$
* \alpha=\mathfrak{s}_{g} \mathrm{~d} \alpha,
$$

where $\mathfrak{s}_{g}=+1$ if $g$ is Riemannian and $\mathfrak{s}_{g}=-1$ if $g$ is Lorentzian.

Remark 2.3. The motivation to introduce the previous definition will be apparent in Section 5, see Theorem 5.4 .

Let $(g, \alpha, \varepsilon)$ be an $\varepsilon$-contact metric structure on $M$ and denote by $\nu_{g}$ the pseudo-Riemannian volume form associated to $g$ and the fixed orientation on $M$. If $g$ is Riemannian then necessarily $\varepsilon=1$ (whence it can be omitted) and $(g, \alpha)$ defines in this case a standard contact metric structure on $M$ [9]. To see this, note that the kernel of $\alpha$ defines an oriented non-integrable rank-two distribution,

$$
\mathcal{D} \stackrel{\text { def. }}{=} \operatorname{ker}(\alpha) \subset T M \text {. }
$$

Denote by $g_{\mathcal{D}}$ the restriction of $g$ to $\mathcal{D}$. Being oriented, the Riemannian volume form of $\left(\mathcal{D}, g_{\mathcal{D}}\right)$ defines a canonical almost complex structure $J_{\mathcal{D}}: \mathcal{D} \rightarrow \mathcal{D}$ with respect to which $\nu_{\mathcal{D}}$ is of type $(1,1)$. Furthermore, the vector field $\xi=\alpha^{\sharp}$ dual to $\alpha$ satisfies by definition $\alpha(\xi)=1$. Equation (2) implies

$$
\mathrm{d} \alpha\left(v_{1}, v_{2}\right)=* \alpha\left(v_{1}, v_{2}\right)=\nu_{\mathcal{D}}\left(v_{1}, v_{2}\right)=g_{\mathcal{D}}\left(v_{1}, J_{\mathcal{D}} v_{2}\right), \quad v_{1}, v_{2} \in \mathcal{D}
$$

Therefore, the tuple $(\mathcal{D}, g, \xi, \phi)$, where $\left.\phi\right|_{\mathcal{D}}=J_{\mathcal{D}}$ and $\phi(\xi)=0$, defines a standard contact metric structure on $M$. Conversely, any such Riemannian contact metric structure gives rise to a canonical Riemannian $\varepsilon$-contact metric structure on $M$. Similar remarks apply when $g$ is Lorentzian and $\varepsilon=-1$, in which case we recover the usual notion of Lorentzian contact metric structure, and when $g$ is Lorentzian and $\varepsilon=1$, in which case we recover the usual notion of para-contact metric structure. Hence the following holds.

Proposition 2.4. Let $M$ be an oriented three-manifold. An $\varepsilon$-contact metric structure $(g, \alpha, \varepsilon)$ on $M$ defines a canonical Riemannian contact metric structure if $g$ is Riemannian, a canonical Lorentzian contact metric structure if $g$ is Lorentzian and $\varepsilon=-1$, and a canonical para-contact metric structure if $g$ is Lorentzian and $\varepsilon=1$. The converse also holds for the three previous cases.

Remark 2.5. By the previous argument, if $(g, \alpha, \varepsilon)$ is an $\varepsilon$-contact metric structure on a Riemannian manifold $(M, g)$, we shall just denote it as a Riemannian contact metric structure in order to keep the usual nomenclature in the literature.

Given an $\varepsilon$-contact structure $(g, \alpha, \varepsilon)$, we will refer to $\xi=\alpha^{\sharp}$ (the metric dual of $\alpha$ ) as the Reeb vector field of $(g, \alpha, \varepsilon)$. The notion of morphism of $\varepsilon$-contact manifolds we consider is the expected one. 
Definition 2.6. Let $\left(M_{a}, g_{a}, \alpha_{a}, \varepsilon_{a}\right), a=1,2$, be $\varepsilon$-contact three-manifolds. A morphism $F$ from $\left(M_{1}, g_{1}, \alpha_{1}, \varepsilon_{1}\right)$ to $\left(M_{2}, g_{2}, \alpha_{2}, \varepsilon_{2}\right)$ with $\varepsilon_{1}=\varepsilon_{2}$ is an orientation preserving smooth map $F: M_{1} \rightarrow M_{2}$ such that:

$$
g_{1}=F^{*} g_{2}, \quad \alpha_{1}=F^{*} \alpha_{2} .
$$

If such $F$ is not orientation preserving, then we will say that it is an orientation-reversing morphism.

Remark 2.7. We denote by PCont the category whose objects are $\varepsilon$-contact three-manifolds and whose morphisms are defined as above. Relevant subcategories of PCont are the subcategory of contact Riemannian three-manifolds $\mathrm{PCont}_{R}$ and the category $\operatorname{PCont}_{L}(\varepsilon)$ of $\varepsilon$-contact Lorentzian three-manifolds with Reeb vector field of norm $\varepsilon \in\{-1,0,1\}$.

In analogy with the standard theory of Riemannian contact structures, we introduce, associated to every $\varepsilon$-contact metric structure $(g, \alpha, \varepsilon)$, two endomorphisms $\phi: T M \rightarrow T M$ and $\mathfrak{h}: T M \rightarrow T M$ :

$$
\phi(v)=-\mathfrak{s}_{g}\left(\iota_{v} * \alpha\right)^{\sharp}, \quad \mathfrak{h}(v)=\left(\mathcal{L}_{\xi} \phi\right)(v) \quad \forall v \in T M,
$$

where $\xi=\alpha^{\sharp}$ is the Reeb vector field of $(g, \alpha, \varepsilon)$ and the symbol $\mathcal{L}$ denotes Lie derivative. We will refer to $\phi \in \Gamma\left(T M \otimes T^{*} M\right)$ as the characteristic endomorphism of $(g, \alpha, \varepsilon)$. Furthermore, from $\phi$ and $\mathfrak{h}$ we define:

$$
\tau \stackrel{\text { def. }}{=} \mathfrak{h} \circ \phi: T M \rightarrow T M
$$

These endomorphisms will play an important role later on. The following lemma summarizes some of the properties enjoyed by $\phi$.

Lemma 2.8. The characteristic endomorphism $\phi$ of an $\varepsilon$-contact metric manifold $(M, g, \alpha, \varepsilon) \in$ PCont satisfies:

$g(\operatorname{Id} \otimes \phi)=\mathrm{d} \alpha, \quad \phi(\xi)=0, \quad \alpha \circ \phi=0, \quad \phi^{2}=\mathfrak{s}_{g}(-\varepsilon \operatorname{Id}+\xi \otimes \alpha), \quad g \circ \phi \otimes \phi=\mathfrak{s}_{g}(\varepsilon g-\alpha \otimes \alpha)$. where $\xi \stackrel{\text { def. }}{=} \alpha^{\sharp}$ denotes the Reeb vector field of $(g, \alpha, \varepsilon)$.

Proof. Using the definition of $\phi$ given in Equation (3), we compute:

$$
g\left(v_{1}, \phi\left(v_{2}\right)\right)=-\mathfrak{s}_{g} g\left(v_{1},\left(\iota_{v_{2}} * \alpha\right)^{\sharp}\right)=-\mathfrak{s}_{g} * \alpha\left(v_{2}, v_{1}\right)=\mathrm{d} \alpha\left(v_{1}, v_{2}\right), \quad v_{1}, v_{2} \in \mathfrak{X}(M),
$$

whence the first equation of the lemma holds. The second and third equations of the lemma follow directly from the definition of $\phi$. On the other hand, the square of the endomorphism $\phi$ can be computed to be:

$$
\begin{aligned}
\phi(\phi(v))=\left[\iota_{\left(\iota_{v} * \alpha\right)^{\sharp}}(* \alpha)\right]^{\sharp} & =\left[(* \alpha)\left(\left(\iota_{v} * \alpha\right)^{\sharp}\right)\right]^{\sharp}=\left[*\left(\alpha \wedge\left(\iota_{v} * \alpha\right)\right)\right]^{\sharp} \\
& =\left[-*\left(\iota_{v}(\alpha \wedge * \alpha)\right)+\alpha(v)(* * \alpha)\right]^{\sharp}=-\mathfrak{s}_{g} \varepsilon v+\mathfrak{s}_{g} \alpha(v) \xi, \quad v \in \mathfrak{X}(M),
\end{aligned}
$$

which implies the fourth equation of the lemma. Finally, this last equation follows from:

$$
g\left(\phi\left(v_{1}\right), \phi\left(v_{2}\right)\right)=\mathrm{d} \alpha\left(\phi\left(v_{1}\right), v_{2}\right)=-\mathrm{d} \alpha\left(v_{2}, \phi\left(v_{1}\right)\right)=-g\left(v_{2}, \phi^{2}\left(v_{1}\right)\right)=\mathfrak{s}_{g} \varepsilon g\left(v_{1}, v_{2}\right)-\mathfrak{s}_{g} \alpha\left(v_{1}\right) \alpha\left(v_{2}\right) .
$$

Remark 2.9. Lemma 2.8 recovers key identities satisfied by $\varepsilon$-contact structures which in classical references are taken as part of the definition of Riemannian contact structures [9] $\left(\varepsilon=\mathfrak{s}_{\mathfrak{g}}=1\right)$, Lorentzian contact structures [15] $\left(\varepsilon=\mathfrak{s}_{\mathfrak{g}}=-1\right)$ or para-contact structures [16] $\left(\varepsilon=-\mathfrak{s}_{\mathfrak{g}}=1\right)$. 
Remark 2.10. Note that the characteristic endomorphism of an $\varepsilon$-contact metric structure $(g, \alpha, \varepsilon)$ is always skew-symmetric with respect to $g$, that is,

$$
g\left(\phi\left(v_{1}\right), v_{2}\right)+g\left(v_{1}, \phi\left(v_{2}\right)\right)=0, \quad \forall v_{1}, v_{2} \in T M .
$$

Given an $\varepsilon$-contact structure $(g, \alpha, \varepsilon)$ we define yet another endomorphism, denoted by $\mathfrak{l} \in \operatorname{End}(T M)$, as follows:

$$
\mathfrak{l}(v)=\mathrm{R}^{g}(v, \xi) \xi, \quad \forall v \in \mathfrak{X}(M),
$$

where $\mathrm{R}^{g}$ denotes the Riemann curvature tensor of $g$. The endomorphism $\mathfrak{l}: T M \rightarrow T M$ should not be confused with the endomorphism defined by the Ricci curvature, which we denote by $\mathrm{Q}^{g}$. This makes five the endomorphisms $\left(\phi, \mathfrak{h}, \tau, \mathfrak{l}, \mathrm{Q}^{g}\right)$ canonically associated to every $\varepsilon$-contact structure $(g, \alpha, \varepsilon)$. Manifolds equipped with an $\varepsilon$-contact structure $(g, \alpha, \varepsilon)$ admit a special frame which is very convenient for computations.

Definition 2.11. Let $(g, \alpha, \varepsilon)$ be an $\varepsilon$-contact metric structure on $M$ with Reeb vector field $\xi \in \mathfrak{X}(M)$. An $\varepsilon$-contact frame is a local frame $\{\xi, u, \phi(u)\}$, where $u \in \mathfrak{X}(M)$ is a nowhere vanishing vector field satisfying:

$$
g(u, u)=\mathfrak{s}_{g} \varepsilon, \quad g(u, \xi)=1-\varepsilon^{2} .
$$

When $\varepsilon=0$ we will refer to $\{\xi, u, \phi(u)\}$ as a light-cone frame for $(g, \alpha, 0)$, following standard usage in Lorentzian geometry.

Remark 2.12. It is a direct calculation to verify that $\{\xi, u, \phi(u)\}$ is indeed a local frame. We have:

$$
g(\xi, \xi)=\varepsilon, \quad g(u, \phi(u))=0, \quad g(\xi, \phi(u))=0, \quad g(\phi(u), \phi(u))=1,
$$

whence $\phi(u)$ is locally nowhere vanishing and point-wise orthogonal to the real span of $\xi$ and $u$.

Proposition 2.13. Let $(g, \alpha, \varepsilon)$ be an $\varepsilon$-contact metric structure. The following equations hold:

$$
\begin{gathered}
\nabla_{\xi} \xi=0, \quad \nabla_{\xi} \phi=0, \quad \mathfrak{h}(\xi)=0, \quad \mathfrak{l}(\xi)=0, \quad \operatorname{Tr}(\mathfrak{h})=0, \\
\mathcal{L}_{\xi} \alpha=0, \quad \operatorname{Tr}(\tau)=0, \quad \mathfrak{h} \circ \phi+\phi \circ \mathfrak{h}=0,
\end{gathered}
$$

where $\nabla$ denotes the Levi-Civita connection with respect to $g$. Furthermore, both $\mathfrak{h}$ and $\tau$ are symmetric with respect to $g$.

Proof. The proof of Equations (4) follows by direct computation on an $\varepsilon$-contact frame. Thus, we prove only the symmetry properties of $\mathfrak{h}$ and $\tau$. We compute:

$$
g\left(\mathfrak{h}\left(v_{1}\right), v_{2}\right)=g\left(\left(\mathcal{L}_{\xi} \phi\right)\left(v_{1}\right), v_{2}\right)=g\left(\mathcal{L}_{\xi} \phi\left(v_{1}\right)-\phi\left(\mathcal{L}_{\xi} v_{1}\right), v_{2}\right)=g\left(-\nabla_{\phi\left(v_{1}\right)} \xi+\phi\left(\nabla_{v_{1}} \xi\right), v_{2}\right) .
$$

This expression vanishes whenever $v_{1}$ or $v_{2}$ are equal to $\xi$. Given an $\varepsilon$-contact frame $\{\xi, u, \phi(u)\}$, assume now that both $v_{1}$ and $v_{2}$ belong to the span of of $u$ and $\phi(u)$. We obtain:

$$
g\left(\mathfrak{h}\left(v_{1}\right), v_{2}\right)=\alpha\left(\nabla_{\phi\left(v_{1}\right)} v_{2}+\nabla_{v_{1}} \phi\left(v_{2}\right)\right)=\alpha\left(\nabla_{\phi\left(v_{2}\right)} v_{1}+\nabla_{v_{2}} \phi\left(v_{1}\right)\right)=g\left(v_{1}, \mathfrak{h}\left(v_{2}\right)\right),
$$

where we have used that $\alpha\left(\left[\phi\left(v_{1}\right), v_{2}\right]\right)+\alpha\left(\left[v_{1}, \phi\left(v_{2}\right)\right]\right)=0$, since

$$
\begin{aligned}
0 & =g\left(\phi\left(v_{1}\right), \phi\left(v_{2}\right)\right)=\mathrm{d} \alpha\left(\phi\left(v_{1}\right), v_{2}\right)-\mathrm{d} \alpha\left(\phi\left(v_{2}\right), v_{1}\right)=\left(\mathcal{L}_{\phi\left(v_{1}\right)} \alpha\right)\left(v_{2}\right)-\left(\mathcal{L}_{\phi\left(v_{2}\right)} \alpha\right)\left(v_{1}\right) \\
& =\alpha\left(\left[\phi\left(v_{1}\right), v_{2}\right]\right)+\alpha\left(\left[v_{1}, \phi\left(v_{2}\right)\right]\right) .
\end{aligned}
$$

The symmetry of $\tau$ follows now directly:

$$
g\left(\tau\left(v_{1}\right), v_{2}\right)=g\left(\mathfrak{h}\left(\phi\left(v_{1}\right)\right), v_{2}\right)=g\left(\phi\left(v_{1}\right), \mathfrak{h}\left(v_{2}\right)\right)=-g\left(v_{1}, \phi\left(\mathfrak{h}\left(v_{2}\right)\right)\right)=g\left(v_{1}, \tau\left(v_{2}\right)\right),
$$

and we conclude. 
An $\varepsilon$-contact structure $(g, \alpha, \varepsilon)$ also satisfies the following identities, which play a key role in the classification of $\varepsilon \eta$-Einstein contact structures.

Proposition 2.14. Let $(g, \alpha, \varepsilon)$ be an $\varepsilon$-contact metric structure. The following equation holds:

$$
2 \phi(\nabla \xi)=\mathfrak{h}+\mathfrak{s}_{g}(\varepsilon-\xi \otimes \alpha),
$$

Remark 2.15. We can apply $\phi$ to the second equation in the previous proposition, obtaining:

$$
2 \phi^{2}(\nabla \xi)=-2 \mathfrak{s}_{g} \varepsilon \nabla^{g} \xi=\mathfrak{s}_{g} \varepsilon \phi+\phi \circ \mathfrak{h} .
$$

For $\varepsilon \neq 0$, that is, for $\varepsilon$-contact structures with non-null Reeb vector field, this equation gives the well-known formula for the covariant derivative of $\xi[9,18]$. For $\varepsilon=0$ this equation reduces to $\tau=0$, which we will prove independently in Lemma 3.3. Therefore, for $\varepsilon$-contact structures with null Reeb vector field, the covariant derivative of the Reeb vector is not prescribed in terms of $\phi$ and $\mathfrak{h}$. This has important consequences in the classification of $\varepsilon \eta$-Einstein $\varepsilon$-contact structures with null Reeb vector field.

Proof. We use Koszul's formula for the Levi-Civita connection:

$$
\begin{gathered}
-2 g\left(\phi\left(\nabla_{v_{1}} \xi\right), v_{2}\right)=2 g\left(\nabla_{v_{1}} \xi, \phi\left(v_{2}\right)\right)=\xi \cdot g\left(v_{1}, \phi\left(v_{2}\right)\right)-\phi\left(v_{2}\right) \cdot \alpha\left(v_{1}\right)-g\left(\mathcal{L}_{\xi} v_{1}, \phi\left(v_{2}\right)\right) \\
+g\left(\left[\phi\left(v_{2}\right), v_{1}\right], \xi\right)-g\left(\mathcal{L}_{\xi}\left(\phi\left(v_{2}\right)\right), v_{1}\right)=-g\left(\mathfrak{h}\left(v_{1}\right), v_{2}\right)-\phi\left(v_{2}\right) \cdot \alpha\left(v_{1}\right)-\alpha\left(\left[v_{1}, \phi\left(v_{2}\right)\right]\right) \\
=-g\left(\mathfrak{h}\left(v_{1}\right), v_{2}\right)+g\left(\phi^{2}\left(v_{1}\right), v_{2}\right)=-g\left(\mathfrak{h}\left(v_{1}\right), v_{2}\right)+g\left(\mathfrak{s}_{g}\left(-\varepsilon v_{1}+\alpha\left(v_{1}\right) \xi\right), v_{2}\right),
\end{gathered}
$$

for every $v_{1}, v_{2} \in \mathfrak{X}(M)$, where we have used $\phi^{2}=\mathfrak{s}_{g}(-\varepsilon \operatorname{Id}+\xi \otimes \alpha)$ and the equation

$$
\mathrm{d} \alpha\left(v_{1}, \phi\left(v_{2}\right)\right)=v_{1} \cdot \alpha\left(\phi\left(v_{2}\right)\right)-\phi\left(v_{2}\right) \cdot \alpha\left(v_{1}\right)-\alpha\left(\left[v_{1}, \phi\left(v_{2}\right)\right]\right)=-\phi\left(v_{2}\right) \cdot \alpha\left(v_{1}\right)-\alpha\left(\left[v_{1}, \phi\left(v_{2}\right)\right]\right) .
$$

Remark 2.16. Note that the equation for $\nabla \xi$ given by Proposition 2.14 differs from the one usually found in the literature by a factor of $\frac{1}{2}$. This discrepancy is due to the different convention we are using for the exterior derivative. Given any $p$-form $\omega$, we define its exterior derivative $\mathrm{d} \omega$ as the $(p+1)$-form given by:

$$
\begin{aligned}
& \mathrm{d} \omega\left(X_{0}, \ldots, X_{p}\right)=\sum_{i}(-1)^{i} X_{i}\left(\omega\left(X_{0}, \ldots, \hat{X}_{i}, \ldots, X_{i}\right)\right) \\
& +\sum_{i<j}(-1)^{i+j} \omega\left(\left[X_{i}, X_{j}\right], X_{0}, \ldots, \hat{X}_{i}, \ldots, \hat{X}_{j}, \ldots, X_{p}\right) .
\end{aligned}
$$

Much of the literature on contact geometry, see for example [9], uses the conventions of Kobayashi and Nomizu [37], in which the formula of the exterior derivative differs by a factor of $\frac{1}{p+1}$ from the one stated above.

Proceeding by analogy with the theory of Riemannian contact metric structures we introduce the notions of Sasakian and K-contact $\varepsilon$-contact metric structures.

Definition 2.17. An $\varepsilon$-contact metric structure $(g, \alpha, \varepsilon)$ is said to be Sasakian if $\mathfrak{h}=0$. It is said to be K-contact if the Reeb vector field is Killing, that is, if $\mathcal{L}_{\xi} g=0$.

Remark 2.18. The Sasakian and K-contact conditions are well-known to be equivalent for $\varepsilon$-contact structures with $\varepsilon \neq 0$ in three dimensions, see $[9,15,16]$. However, as we will see in Section 3, this fails to be the case for null contact metric structures. Indeed, Example 3.17 shows that the 
theory of Sasakian null contact metric structures is strictly richer that the theory of null K-contact structures.

Sasakian $\varepsilon$-contact metric structures with $\varepsilon \neq 0$ have been extensively studied in the literature, see for instance [9], [15] or [54] for more details and an exhaustive list of references. In particular, it is well-known that the Sasakian condition can sometimes be equivalently formulated as a curvature condition involving the Ricci curvature tensor $\mathrm{Ric}^{g}$. Since this will be of use later, we briefly review this result using our conventions.

Proposition 2.19. Let $(g, \alpha, \varepsilon)$ be an $\varepsilon$-contact structure with $\varepsilon \neq 0$. Then

$$
\operatorname{Ric}^{g}(\xi, \xi)=\varepsilon \mathfrak{s}_{\mathfrak{g}}\left(\frac{1}{2}-\frac{1}{4} \operatorname{Tr}\left(\mathfrak{h}^{2}\right)\right) .
$$

where $\operatorname{Ric}^{g}$ is the Ricci curvature of $g$.

Proof. The proofs of the proposition in the Riemannian and Lorentzian $\varepsilon=-1$ cases are presented in detail in [9] and [15], respectively. Hence we focus on the para-contact case.

Proposition 2.14 adapted to the para-contact case yields $\nabla \xi=-\frac{1}{2} \phi+\frac{1}{2} \phi \circ \mathfrak{h}$. Hence, denoting by $\mathrm{R}^{g}$ the Riemann curvature tensor, we have:

$\mathrm{R}^{g}(\xi, v) \xi=\nabla_{\xi} \nabla_{v} \xi-\nabla_{v} \nabla_{\xi} \xi-\nabla_{[\xi, v]} \xi=-\frac{1}{2} \nabla_{\xi}(\phi(v))+\frac{1}{2} \nabla_{\xi}(\phi(\mathfrak{h}(v)))+\frac{1}{2} \phi([\xi, v])-\frac{1}{2} \phi(\mathfrak{h}([\xi, v]))$,

for $v \in \operatorname{ker}(\alpha)$ and where we used that $\nabla_{\xi} \xi=0$. Applying $\phi$ at the previous equation, taking into account that $\phi^{2}=\mathrm{Id}-\xi \otimes \alpha$ and that $\nabla_{\xi} \phi=0$, we obtain:

$$
\left.\phi\left(\mathrm{R}^{g}(\xi, v) \xi\right)=\frac{1}{2} \nabla_{\xi}(-v+\mathfrak{h}(v))+\frac{1}{2}[\xi, v]-\frac{1}{2} \mathfrak{h}([\xi, v])\right),
$$

where we have used that $\alpha \circ \phi=\alpha \circ \mathfrak{h}=0$ and that $\alpha([\xi, v])=0$ (the latter equation follows from the para-contact condition $\mathrm{d} \alpha=-* \alpha$ ). Applying now Proposition 2.14, we conclude:

$$
\phi\left(\mathrm{R}^{g}(\xi, v) \xi\right)=\frac{1}{2}\left(\nabla_{\xi} \mathfrak{h}\right)(v)+\frac{1}{4} \phi(v)-\frac{1}{4} \mathfrak{h}^{2}(\phi(v)) .
$$

On the other hand, $\phi^{2}\left(\mathrm{R}^{g}(\xi, v) \xi\right)=\mathrm{R}^{g}(\xi, v) \xi$ since $\alpha\left(\mathrm{R}^{g}(\xi, v) \xi\right)=0$ vanishes identically. Consequently,

$$
\mathrm{R}^{g}(\xi, v) \xi=\frac{1}{2} \phi\left(\nabla_{\xi} \mathfrak{h}\right)(v)+\frac{1}{4} \phi^{2}(v)-\frac{1}{4} \mathfrak{h}^{2}(v)
$$

Equation (5) implies that

$$
\phi\left(\mathrm{R}^{g}(\xi, \phi(v)) \xi\right)=-\frac{1}{2} \phi\left(\nabla_{\xi} \mathfrak{h}\right)(v)+\frac{1}{4} \phi^{2}(v)-\frac{1}{4} \mathfrak{h}^{2}(v) .
$$

Using now the previous formulae for $v$ of unit norm we obtain:

$$
g\left(\mathrm{R}^{g}(\xi, v) \xi, v\right)+g\left(\phi\left(\mathrm{R}^{g}(\xi, \phi(v)) \xi\right), v\right)=\frac{1}{2}-\frac{1}{4} \operatorname{Tr}\left(\mathfrak{h}^{2}\right)=-\mathrm{Ric}^{g}(\xi, \xi),
$$

where we used the fact that $g\left(\mathfrak{h}^{2} \phi(v), v\right)=-g\left(\mathfrak{h}^{2}(v), v\right)$, so $g\left(\mathfrak{h}^{2}(v), v\right)=\frac{1}{2} \operatorname{Tr}\left(\mathfrak{h}^{2}\right)$. Hence

$$
\operatorname{Ric}^{g}(\xi, \xi)=-\frac{1}{2}+\frac{1}{4} \operatorname{Tr}\left(\mathfrak{h}^{2}\right),
$$

and we conclude. 
Remark 2.20. The equation proved in Proposition 2.19 differs from the one found usually in the literature by a factor of $\frac{1}{4}$. This difference can be traced back to the different convention used in this work for the exterior derivative and $\mathfrak{h}$ with respect to the conventions used in references [9] and [15].

Proposition 2.21. An $\varepsilon$-contact structure $(g, \alpha, \varepsilon)$ with $\varepsilon \mathfrak{s}_{g}=1$ is Sasakian if and only if:

$$
\operatorname{Ric}^{g}(\xi, \xi)=\mathfrak{s}_{g} \frac{\varepsilon}{2} .
$$

Proof. The only if direction follows by setting $\mathfrak{h}=0$ in Proposition 2.19. Conversely, if $\operatorname{Ric}^{g}(\xi, \xi)=$ $\mathfrak{s}_{g} \frac{\varepsilon}{2}$ then Proposition 2.19 implies that $\operatorname{Tr}\left(\mathfrak{h}^{2}\right)=0$. Taking into account that $\phi$ is skew-adjoint with respect to $g$ and that $\mathfrak{h}$ is self-adjoint with respect to $g$, we obtain $g\left(\mathfrak{h}^{2}(X), \phi(X)\right)=0$ for every vector field $X$. Since $\mathfrak{h}(\xi)=0$ and $g$ is positive definite when restricted to $\operatorname{ker}(\alpha)$ (assuming $\varepsilon_{\mathfrak{s}_{g}}=1$ ), then condition $\operatorname{Tr}\left(\mathfrak{h}^{2}\right)=0$ implies that $\mathfrak{h}^{2}=0$. Since $\mathfrak{h}$ is self-adjoint, this yields $\mathfrak{h}=0$.

2.1. Globally hyperbolic $\varepsilon$-contact metric three-manifolds. In this section we describe $\varepsilon$ contact metric structures on globally hyperoblic Lorentzian manifolds. This class of $\varepsilon$-contact metric three-manifolds is specially relevant for our purposes, since they can be used to construct globally hyperbolic Lorentzian six-manifolds equipped with a Ricci-flat metric connection with totally skew-symmetric and closed torsion, as described in Section 5 and Theorem 5.4. At the same time, globally hyperbolic solutions of six-dimensional supergravity play a prominent role in the celebrated fuzzball proposal to describe the microscopic entropy of a black hole, see $[6,43]$ and references therein for more details.

Let $(M, g)$ be a globally hyperbolic Lorentzian three-manifold. A celebrated theorem of A. Bernal and M. Sánchez $[8]$ states that $(M, g)$ admits the following presentation:

$$
(M, g)=\left(\mathbb{R} \times \mathrm{X},-\beta_{t}^{2} \mathrm{~d} t \otimes \mathrm{d} t+q_{t}\right),
$$

where $t$ is a coordinate on $\mathbb{R},\left\{\beta_{t}\right\}_{t \in \mathbb{R}}$ is a family of nowhere vanishing functions on $\mathrm{X} \stackrel{\text { def. }}{=}\{0\} \times \mathrm{X}$ and $\left\{q_{t}\right\}$ is a family of complete Riemannian metrics on $X$. In this presentation, $(M, g)$ is oriented and time-oriented, which immediately fixes an orientation on $\mathrm{X}$. We denote by $\nu_{q_{t}}$ the Riemannian volume form associated to $q_{t}$. Let $\alpha$ be a one form on $M$. Set $e_{t}^{0}=\beta_{t} \mathrm{~d} t$. We write:

$$
\alpha=F_{t} e^{0}+\alpha_{t}^{\perp},
$$

where $\left\{F_{t}\right\}_{t \in \mathbb{R}}$ is a unique family of nowhere vanishing functions on $\mathrm{X}$ and $\left\{\alpha_{t}\right\}_{t \in \mathbb{R}}$ is a unique family of one-forms on $X$. With these provisos in mind, the dual of $\alpha$ can be computed to be:

$$
* \alpha=-F_{t} \nu_{q_{t}}-e^{0} \wedge *_{q_{t}} \alpha_{t}^{\perp}
$$

where $*_{q_{t}}$ denotes the Hodge-dual of $\left(X, q_{t}\right)$. On the other hand, the exterior derivative of $\alpha$ reads:

$$
\mathrm{d} \alpha=\mathrm{d}_{\mathrm{X}} F_{t} \wedge e_{t}^{0}+\frac{F_{t}}{\beta_{t}} \mathrm{~d}_{\mathrm{X}} \beta_{t} \wedge e_{t}^{0}+\frac{1}{\beta_{t}} e_{t}^{0} \wedge \partial_{t} \alpha_{t}^{\perp}+\mathrm{d}_{\mathrm{X}} \alpha_{t}^{\perp},
$$

where $\mathrm{d}_{\mathrm{X}}$ is the exterior derivative on $\mathrm{X}$. Define $n_{t} \stackrel{\text { def. }}{=} \frac{1}{\beta_{t}} \partial_{t}$. Altogether, the previous discussion implies the following characterization of $\varepsilon$-contact structures on $(M, g)$.

Proposition 2.22. Let $(M, g)=\left(\mathbb{R} \times \mathrm{X},-\beta_{t}^{2} \mathrm{~d} t \otimes \mathrm{d} t+q_{t}\right)$ be a globally hyperbolic Lorentzian three-manifold. A one-form $\alpha \in \Omega^{1}(M)$ defines an $\varepsilon$-contact metric structure on $(M, g)$ if and only if:

$$
\mathrm{d}_{\mathrm{X}} \alpha_{t}^{\perp}=F_{t} \nu_{q_{t}}, \quad *_{q_{t}} \alpha_{t}^{\perp}+\frac{1}{\beta_{t}} \mathrm{~d}_{\mathrm{X}}\left(\beta_{t} F_{t}\right)=\mathcal{L}_{n_{t}} \alpha_{t}^{\perp}, \quad\left|\alpha_{t}^{\perp}\right|_{q_{t}}^{2}=\varepsilon+F_{t}^{2}
$$


where $\left\{F_{t}\right\}_{t \in \mathbb{R}},\left\{\beta_{t}\right\}$ and $\left\{\alpha_{t}^{\perp}\right\}_{t \in \mathbb{R}}$ are defined above and $\alpha=F_{t} e^{0}+\alpha_{t}^{\perp}$.

Remark 2.23. The previous proposition yields a system of flow equations for a pair of families of functions $\left\{F_{t}\right\}_{t \in \mathbb{R}},\left\{\beta_{t}\right\}_{t \in \mathbb{R}}$, a family of one-forms $\left\{\alpha_{t}^{\perp}\right\}_{t \in \mathbb{R}}$ and a family of complete Riemannian metrics $\left\{q_{t}\right\}_{t \in \mathbb{R}}$ on an oriented two-manifold $\mathrm{X}$. To the best of our knowledge, this system has not been studied in the literature. We hope to study it in more detail elsewhere.

Definition 2.24. Given a three-manifold $M=\mathbb{R} \times X$, a globally hyperbolic $\varepsilon$-contact structure on $M$ is a tuple

$$
\left(\left\{\beta_{t}\right\}_{t \in \mathbb{R}},\left\{F_{t}\right\}_{t \in \mathbb{R}},\left\{\alpha_{t}^{\perp}\right\}_{t \in \mathbb{R}},\left\{q_{t}\right\}_{t \in \mathbb{R}}\right)
$$

defined as specified in Remark 2.23, which satisfies Equations 6.

Given a globally hyperbolic $\varepsilon$-contact structure $\left(\left\{\beta_{t}\right\}_{t \in \mathbb{R}},\left\{F_{t}\right\}_{t \in \mathbb{R}},\left\{\alpha_{t}^{\perp}\right\}_{t \in \mathbb{R}},\left\{q_{t}\right\}_{t \in \mathbb{R}}\right)$, it is clear from the previous discussion how to reconstruct $(g, \alpha, \varepsilon)$. We consider now a particular example in the case $\varepsilon=1$.

Example 2.25. In the conditions of Proposition 2.22 set

$$
F_{t}=0, \quad \beta_{t}=1 .
$$

With these choices for $\left\{F_{t}\right\}_{t \in \mathbb{R}}$ and $\left\{\beta_{t}\right\}_{t \in \mathbb{R}}$, Equations (6) read:

$$
\mathrm{d}_{\mathrm{X}} \alpha_{t}^{\perp}=0, \quad *_{q_{t}} \alpha_{t}^{\perp}=\partial_{t} \alpha_{t}^{\perp}, \quad\left|\alpha_{t}^{\perp}\right|_{q_{t}}^{2}=\varepsilon,
$$

which immediately implies $\varepsilon=1$, corresponding to the case in which the Reeb vector field is space-like and the associated pair $(g, \alpha, \varepsilon=1)$ defines a para-contact metric structure. Consider now

$$
\left(\mathrm{X}, q_{t}\right)=\left(\mathbb{R}^{2}, e^{2 U_{t}}(\mathrm{~d} x \otimes \mathrm{d} x+\mathrm{d} y \otimes \mathrm{d} y)\right),
$$

where $\left\{U_{t}\right\}_{t \in \mathbb{R}}$ is a family of constant functions and $(x, y)$ are coordinates on $\mathbb{R}^{2}$. Let us write $\alpha^{\perp}=e^{U_{t}}\left(\alpha_{1}^{\perp} \mathrm{d} x+\alpha_{2}^{\perp} \mathrm{d} y\right)$. With these assumptions, Equations (7) are equivalent to:

$$
\partial_{t}\left(\alpha_{1}^{\perp} e^{U_{t}}\right)=-\alpha_{2}^{\perp} e^{U_{t}}, \quad \partial_{t}\left(\alpha_{2}^{\perp} e^{U_{t}}\right)=\alpha_{1}^{\perp} e^{U_{t}}, \quad\left(\alpha_{1}^{\perp}\right)^{2}+\left(\alpha_{2}^{\perp}\right)^{2}=1 .
$$

These equations are solved by:

$$
\alpha_{1}^{\perp} e^{U_{t}}=l_{2} \cos (t)-l_{1} \sin (t), \quad \alpha_{2}^{\perp} e^{U_{t}}=l_{1} \cos (t)+l_{2} \sin (t), \quad e^{2 U_{t}}=l_{1}^{2}+l_{2}^{2},
$$

for real constants $l_{1}$ and $l_{2}$ such that $l_{1}^{2}+l_{2}^{2} \neq 0$.

\section{Null CONTACT Metric Structures}

The case of $\varepsilon$-contact metric structures with null Reeb vector field $(\varepsilon=0)$ seems to be new in the literature and thus deserves further attention. For simplicity in the exposition, we will refer to Lorentzian $\varepsilon$-contact metric structures with $\varepsilon=0$ simply as null contact structures. The definition of null contact metric structure allows for the Reeb vector field to be identically zero. We will refer to this case as being trivial. Unless otherwise specified, we will always consider non-trivial null contact metric structures.

Remark 3.1. Let $(g, \alpha)$ be a null contact structure. We have:

$$
\alpha \wedge \mathrm{d} \alpha=-\alpha \wedge * \alpha=0,
$$

where we have used that $|\alpha|_{g}^{2}=0$. Therefore, the previous computation implies that the one-form $\alpha$ of a null contact structure is not a contact form since it does not satisfy $\alpha \wedge \mathrm{d} \alpha \neq 0$. Nevertheless, given that the definition of $\varepsilon$-contact structure encompasses Riemannian contact $(\varepsilon=1)$, Lorentzian 
contact $(\varepsilon=-1)$ and para-contact $(\varepsilon=1)$ metric structures, we interpret the remaining case $\varepsilon=0$ as a natural generalization of the formers but in which the Reeb vector field is null. Hence we will continue referring to the case $\varepsilon=0$ as a null contact structure. Moreover, we will show later in this section that null contact structures admit meaningful notions of Sasakianity and K-contactness, analogously to the $\varepsilon \neq 0$ cases.

Having shed some light into the nature of null contact structures, we proceed to investigate their most relevant properties. We recall that Lemma 2.8 applied to a null contact structure $(g, \alpha)$ implies:

$$
\phi^{2}=-\xi \otimes \alpha,
$$

from which the following lemma follows.

Lemma 3.2. The characteristic endomorphism $\phi$ of a null contact metric structure $(g, \alpha)$ satisfies

$$
\phi^{3}=0 \text {. }
$$

Hence $\phi$ is nilpotent.

In the null contact case, the tensor field $\mathfrak{h} \stackrel{\text { def. }}{=} \mathcal{L}_{\xi} \phi$ satisfies additional properties not listed in Proposition 2.14 (compare also with Remark 2.15).

Lemma 3.3. Let $(g, \alpha)$ be a null contact structure. Then $\phi \circ \mathfrak{h}=\mathfrak{h} \circ \phi=0$.

Proof. Let $v_{1}, v_{2} \in T M$. Combining the first and fifth equations in Lemma 2.8, we have

$$
\mathrm{d} \alpha\left(v_{1}, \phi\left(v_{2}\right)\right)=-\alpha\left(v_{1}\right) \alpha\left(v_{2}\right) .
$$

Applying the Lie derivative along the Reeb vector field $\xi$ to both sides of the previous equation, we obtain:

$$
\mathrm{d} \alpha\left(\mathcal{L}_{\xi} v_{1}, \phi\left(v_{2}\right)\right)+\mathrm{d} \alpha\left(v_{1}, \mathfrak{h}\left(v_{2}\right)\right)+\mathrm{d} \alpha\left(v_{1}, \phi\left(\mathcal{L}_{\xi} v_{2}\right)\right)=-\alpha\left(\mathcal{L}_{\xi} v_{1}\right) \alpha\left(v_{2}\right)-\alpha\left(v_{1}\right) \alpha\left(\mathcal{L}_{\xi} v_{2}\right),
$$

whence

$$
\mathrm{d} \alpha\left(v_{1}, \mathfrak{h}\left(v_{2}\right)\right)=0
$$

which by the first equation in Lemma 2.8 is equivalent to

$$
g\left(v_{1}, \phi \circ \mathfrak{h}\left(v_{2}\right)\right)=0,
$$

for every $v_{1}, v_{2} \in T M$. Hence $\phi \circ \mathfrak{h}=0$, which, combined with Proposition 2.13 implies $\mathfrak{h} \circ \phi=0$ and we conclude.

Proposition 3.4. The tensor field $\mathfrak{h}$ associated to a null contact structure $(g, \alpha)$ over $M$ can always be written as:

$$
\mathfrak{h}=\mu \xi \otimes \alpha,
$$

for a function $\mu \in C^{\infty}(M)$.

Remark 3.5. The previous Proposition implies that $\mathfrak{h}$ cannot have non-zero eigenvalues, in notable contrast with the situation occurring when $\varepsilon \neq 0[9,15,53]$, where certain eigenbundles of $\mathfrak{h}$ with non-zero eigenvalue play a crucial role in the classification of $\varepsilon \eta$-Einstein and $(\kappa, \mu)$ contact metric three-manifolds $[9,17]$. 
Proof. Choose a light-cone frame $\{\xi, u, \phi(u)\}$. By Lemma 2.14, $\phi(\xi)=0$ hence $\xi \in \operatorname{ker}(\phi)$. By Remark 2.12 we have $g(\phi(u), \phi(u))=1$, whence $u \notin \operatorname{ker}(\phi)$. Furthermore, $\phi^{2}(u)=-\xi$, so $\phi(u) \notin$ $\operatorname{ker}(\phi)$ and we conclude that $\operatorname{ker}(\phi)=\operatorname{Span}_{C^{\infty}}\{\xi\}$. By Lemma 3.3 we have $\phi \circ \mathfrak{h}=0$, and thus $\operatorname{Im}(\mathfrak{h}) \subseteq \operatorname{ker}(\phi)$, whence

$$
\mathfrak{h}=\gamma \otimes \xi
$$

for a certain one-form $\gamma$. Since $\mathfrak{h}(\xi)=0$ (see Proposition 2.14) we conclude that $\gamma=\mu \alpha+c \phi(u)^{b}$ for some functions $\mu$ and $c$. On the other hand, by Lemma 3.3 we have $\mathfrak{h} \circ \phi=0$, which is equivalent to $\gamma \circ \phi=0$. This implies that $c=0$ after evaluating at $u$, that is, imposing $\gamma(\phi(u))=0$.

Remark 3.6. Expressed in a light-cone frame $\{\xi, u, \phi(u)\}$, the endomorphism $\mathfrak{h}$ has the following point-wise matrix form:

$$
\mathfrak{h}=\left[\begin{array}{lll}
0 & \mu & 0 \\
0 & 0 & 0 \\
0 & 0 & 0
\end{array}\right],
$$

whence it has a unique type as an endomorphism of the tangent space. This shall be compared with the results of [18] where, in the para-contact case, the possible types of $\mathfrak{h}$ in an orthonormal special basis were classified.

The additional properties satisfied by the tensor field $\mathfrak{h}$ allows us to obtain an explicit expression for the Lie brackets of a light-cone frame $\{\xi, u, \phi(u)\}$.

Lemma 3.7. Let $(g, \alpha)$ be a null contact structure over $M$. For a light-cone frame $\{\xi, u, \phi(u)\}$ we have:

$$
[\xi, u]=b \xi+c \phi(u), \quad[\xi, \phi(u)]=(\mu-c) \xi, \quad[u, \phi(u)]=e \xi+u+f \phi(u),
$$

where $\mu=g(u, \mathfrak{h}(u))$ and $b, c, e$ and $f$ are local functions.

Proof. Equation $\mathcal{L}_{\xi} \alpha=0$ implies that $\alpha([\xi, u])=0$, whence $[\xi, u]=b \xi+c \phi(u)$. Therefore:

$$
[\xi, \phi(u)]=\phi([\xi, u])+\mathfrak{h}(u)=-c \xi+\mu \xi=(\mu-c) \xi .
$$

Furthermore, by the $\varepsilon$-contact structure condition $* \alpha=-\mathrm{d} \alpha$, we deduce that $\alpha([u, \phi(u)])=1$, so necessarily $[u, \phi(u)]=e \xi+u+f \phi(u)$.

The following examples show that null contact metric structures are abundant.

Example 3.8. Take $M=\mathbb{R}^{3}$ and fix $g=\delta$ to be the standard Minkowski metric of signature $(-,+,+)$ in $\mathbb{R}^{3}$ with orthonormal coordinates $(t, x, y), t$ being time-like. Then:

$$
\delta=-\mathrm{d} t \otimes \mathrm{d} t+\mathrm{d} x \otimes \mathrm{d} x+\mathrm{d} y \otimes \mathrm{d} y, \quad \alpha=e^{y}(\mathrm{~d} t-\mathrm{d} x)
$$

is a null contact metric structure. The characteristic endomorphism of this null contact structure can be found to be

$$
\phi=e^{y} \partial_{y} \otimes \mathrm{d} t-e^{y} \partial_{y} \otimes \mathrm{d} x+e^{y}\left(\partial_{t}+\partial_{x}\right) \otimes \mathrm{d} y .
$$

A direct computation shows that:

$$
\phi^{2}=e^{2 y}\left(\partial_{t}+\partial_{x}\right) \otimes \mathrm{d} t-e^{2 y}\left(\partial_{t}+\partial_{x}\right) \otimes \mathrm{d} x=-\xi \otimes \alpha, \quad \phi^{3}=0,
$$

as expected. The Reeb vector field is given by $\xi=\alpha^{\sharp}=-e^{y}\left(\partial_{t}+\partial_{x}\right)$ and we have

$$
\mathcal{L}_{\xi} \delta=e^{y}(\mathrm{~d} t \odot \mathrm{d} y-\mathrm{d} x \odot \mathrm{d} y) .
$$


Hence $(\delta, \alpha)$ is not K-contact, and by Proposition 3.18 below, it cannot be Sasakian either. On the other hand, the endomorphism $\mathfrak{h}$ can be found to be:

$$
\mathfrak{h} \stackrel{\text { def. }}{=} \mathcal{L}_{\xi} \phi=-\xi \otimes \alpha,
$$

in agreement with Proposition 3.4 with $\mu=-1$.

Example 3.9. Consider the $M=\widetilde{\mathrm{Sl}}(2, \mathbb{R})$, where $\widetilde{\mathrm{S}}(2, \mathbb{R})$ denotes the universal cover group of $\mathrm{Sl}(2, \mathbb{R})$. There exists a Lorentzian metric $g$ with left-invariant orthonormal global frame $\left\{e^{0}, e^{1}, e^{2}\right\}$ on $\widetilde{\mathrm{Sl}}(2, \mathbb{R})$ satisfying [49]:

$$
\mathrm{d} e^{0}=e^{1} \wedge e^{2}, \quad \mathrm{~d} e^{1}=e^{0} \wedge e^{2}, \quad \mathrm{~d} e^{2}=-e^{0} \wedge e^{1}
$$

and whose associated Lorentzian metric $g$ is given by:

$$
g=-e^{0} \otimes e^{0}+e^{1} \otimes e^{1}+e^{2} \otimes e^{2} .
$$

Hence $* e^{0}=-e^{1} \wedge e^{2}, * e^{1}=-e^{0} \wedge e^{2}$ and $* e^{2}=e^{0} \wedge e^{1}$. We take $\alpha$ to be a null and left-invariant one-form on $M$ and we expand it the basis $\left\{e^{0}, e^{1}, e^{2}\right\}$,

$$
\alpha=\alpha_{0} e^{0}+\alpha_{1} e^{1}+\alpha_{2} e^{2},
$$

for some real constant coefficients $\left\{\alpha_{a}\right\}, a=0,1,2$, satisfying $\alpha_{0}^{2}=\alpha_{1}^{2}+\alpha_{2}^{2}$. We compute:

$$
\mathrm{d} \alpha=\alpha_{0} e^{1} \wedge e^{2}+\alpha_{1} e^{0} \wedge e^{2}-\alpha_{2} e^{0} \wedge e^{1}=-* \alpha .
$$

Therefore, every null left-invariant one-form on $\widetilde{\mathrm{Sl}}(2, \mathbb{R})$ equipped with the Lorentzian metric $g$ defines a null contact structure $(g, \alpha)$. We obtain now the characteristic endomorphism $\phi$ of $(g, \alpha)$ as well as its square. A direct computation yields the following matrix representation for $\phi$ in the basis $\left\{e^{0}, e^{1}, e^{2}\right\}$ :

$$
\phi=\left[\begin{array}{ccc}
0 & \alpha_{2} & -\alpha_{1} \\
\alpha_{2} & 0 & \alpha_{0} \\
-\alpha_{1} & -\alpha_{0} & 0
\end{array}\right]
$$

For the square and cubic powers of $\phi$ we obtain:

$$
\phi^{2}=\left[\begin{array}{ccc}
\alpha_{0}^{2} & \alpha_{0} \alpha_{1} & \alpha_{0} \alpha_{2} \\
-\alpha_{0} \alpha_{1} & -\alpha_{1}^{2} & -\alpha_{1} \alpha_{2} \\
-\alpha_{0} \alpha_{2} & -\alpha_{1} \alpha_{2} & -\alpha_{2}^{2}
\end{array}\right]=-[\xi \otimes \alpha], \quad \phi^{3}=0,
$$

as expected from Lemma 3.2 .

Remark 3.10. By the results of [27, 29], see in particular Theorem A in [29], Examples 3.8 and 3.9 show that all closed Lorentzian three-manifolds admitting a non-compact isometry group carry null contact structures.

We generalize now Example 3.9 by classifying all simply-connected, Lorentzian, three-dimensional Lie groups admitting left-invariant null contact structures. For this, we will exploit the classification of this type of groups available in the literature [17, 24, 49], which we summarize for completeness in Appendix A.

Proposition 3.11. A three-dimensional connected and simply connected Lie group $\mathrm{G}$ admits a left-invariant null contact structure $(g, \alpha)$ if and only if $(\mathrm{G}, g, \alpha)$ is isomorphic, through a possibly orientation-reversing isometry, to one of the items listed in the following table in terms of the orthonormal frame $\left\{e_{0}, e_{1}, e_{2}\right\}$ appearing in Theorem A.1: 


\begin{tabular}{|c|c|c|c|}
\hline $\mathfrak{g}$ & Structure constants $\left(s \in \mathbb{Z}_{2}\right)$ & $\alpha$ & $\mathrm{G}$ \\
\hline $\mathfrak{g}_{1}$ & $a \neq 0, b=s$ & $\alpha_{1}=0, \alpha_{0}=-\alpha_{2}$ & $\widetilde{\mathrm{Sl}}(2, \mathbb{R})$ \\
\hline \multirow{4}{*}{$\mathfrak{g}_{3}$} & $a \neq 0, b=c=s$ & $\alpha_{1}=0, \alpha_{0}^{2}=\alpha_{2}^{2}$ & $\widetilde{\mathrm{Sl}}(2, \mathbb{R})$ \\
\hline & $a=c=s, b \neq 0$ & \multirow{2}{*}{$\alpha_{2}=0, \alpha_{0}^{2}=\alpha_{1}^{2}$} & $\widetilde{\mathrm{Sl}}(2, \mathbb{R})$ \\
\hline & $a=s, b=0, c=s$ & & $\widetilde{\mathrm{E}}(1,1)$ \\
\hline & $a=b=c=s$ & $\alpha_{0}^{2}=\alpha_{1}^{2}+\alpha_{2}^{2}, \alpha_{1}, \alpha_{2} \neq 0$ & $\widetilde{\mathrm{Sl}}(2, \mathbb{R})$ \\
\hline \multirow{2}{*}{$\mathfrak{g}_{4}$} & $b=s+\mu, a \neq 0, \mu \in \mathbb{Z}_{2}$ & \multirow{2}{*}{$\alpha_{1}=0, \alpha_{0}=\mu \alpha_{2}$} & $\widetilde{\mathrm{Sl}}(2, \mathbb{R})$ \\
\hline & $b=s+\mu, a=0, \mu \in \mathbb{Z}_{2}$ & & $\widetilde{\mathrm{E}}(1,1)$ \\
\hline $\mathfrak{g}_{6}$ & $\begin{array}{c}\mu a=(b+s), \mu d=(c+s) \\
b=c, a=d \neq 0\end{array}$ & $\alpha_{1}=0, \alpha_{0}=-\mu \alpha_{2}$ & $\mathfrak{G}_{6}$ \\
\hline
\end{tabular}

Proof. Every connected and simply-connected three-dimensional Lorentzian Lie group is isometric to one of the items listed in Theorem A.1 through an isometry which may be orientation-reversing. Therefore we proceed on a case by case basis by solving the equation

$$
* \alpha=-s \mathrm{~d} \alpha, \quad s \in\{-1,+1\}
$$

in each of the cases listed in the table appearing in Theorem A.1. The sign $s$ is introduced because the $\varepsilon$-contact condition is not invariant under orientation reversing morphisms. If a solution is found with $s=-1$ then an orientation reversing isometry yields an isomorphic Lorentzian Lie algebra admitting a null-contact structure satisfying equation $* \alpha=-\mathrm{d} \alpha$. Let $\left\{e_{0}, e_{1}, e_{2}\right\}$ be the frame appearing in Theorem A.1 and let $\left\{e^{0}, e^{1}, e^{2}\right\}$ be its dual frame. We fix the volume form to be $\nu=e^{0} \wedge e^{1} \wedge e^{2}$. For the rest of the proof we will write:

$$
\alpha=\alpha_{0} e^{0}+\alpha_{1} e^{1}+\alpha_{2} e^{2},
$$

where $\alpha_{0}, \alpha_{1}$ and $\alpha_{2}$ are real coefficients satisfying $\alpha_{0}^{2}=\alpha_{1}^{2}+\alpha_{2}^{2}$ (hence $\alpha_{0} \neq 0$ in order for $\alpha$ to be non-zero). If $\left\{e^{0}, e^{1}, e^{2}\right\}$ denotes the dual co-frame, we compute:

$$
* \alpha=-\alpha_{0} e^{1} \wedge e^{2}-\alpha_{1} e^{0} \wedge e^{2}+\alpha_{2} e^{0} \wedge e^{1} .
$$

- Case $\mathfrak{g}_{1}$. In the orthonormal frame for $\mathfrak{g}_{1}$ given in Appendix A, Equation $* \alpha=-s \mathrm{~d} \alpha$ is equivalent to

$$
\begin{aligned}
\left(-a \alpha_{1}-b \alpha_{2}\right) e^{0} & \wedge e^{1}+\left(a \alpha_{0}+b \alpha_{1}+a \alpha_{2}\right) e^{0} \wedge e^{2}+\left(b \alpha_{0}-a \alpha_{1}\right) e^{1} \wedge e^{2} \\
= & s\left(\alpha_{0} e^{1} \wedge e^{2}+\alpha_{1} e^{0} \wedge e^{2}-\alpha_{2} e^{0} \wedge e^{1}\right),
\end{aligned}
$$

A solution $(a, b)$ exists for a non-zero $\alpha$ if and only if $b=s$. Using that $a \neq 0$ by Theorem A, if $b=s$ we obtain $\alpha_{0}=-\alpha_{2}$, so $\alpha_{1}=0$.

- Case $\mathfrak{g}_{2}$. In the orthonormal frame for $\mathfrak{g}_{2}$ given in Appendix A, Equation $* \alpha=-s \mathrm{~d} \alpha$ is equivalent to

$$
\begin{gathered}
a \alpha_{1} e^{0} \wedge e^{2}+\left(c \alpha_{0}-b \alpha_{2}\right) e^{0} \wedge e^{1}+\left(c \alpha_{2}+b \alpha_{0}\right) e^{1} \wedge e^{2} \\
=s \alpha_{0} e^{1} \wedge e^{2}+s \alpha_{1} e^{0} \wedge e^{2}-s \alpha_{2} e^{0} \wedge e^{1}
\end{gathered}
$$


The previous equations imply the condition $c^{2}+(b-s)^{2}=0$, whose unique solution is $c=0$ and $b=s$. However the value $c=0$ is forbidden for $\mathfrak{g}_{2}$ algebras, so no null contact structures exist on this type of algebras.

- Case $\mathfrak{g}_{3}$. In the orthonormal frame for $\mathfrak{g}_{3}$ given in Appendix A, Equation $* \alpha=-s \mathrm{~d} \alpha$ is equivalent to

$$
c \alpha_{0} e^{1} \wedge e^{2}+a \alpha_{1} e^{0} \wedge e^{2}-b \alpha_{2} e^{0} \wedge e^{1}=s \alpha_{0} e^{1} \wedge e^{2}+s \alpha_{1} e^{0} \wedge e^{2}-s \alpha_{2} e^{0} \wedge e^{1} .
$$

Since $\alpha_{0} \neq 0$ if $\alpha$ is non-vanishing, we have $c=s$. If $\alpha_{1}=0$, then $\alpha_{0}^{2}=\alpha_{2}^{2}$ and $b=s$. If $\alpha_{2}=0$, then $\alpha_{0}^{2}=\alpha_{1}^{2}$ and $a=s$. If $\alpha_{1}, \alpha_{2} \neq 0$, then $a=b=s$.

- Case $\mathfrak{g}_{4}$. In the orthonormal frame for $\mathfrak{g}_{4}$ given in Appendix A, Equation $* \alpha=-s \mathrm{~d} \alpha$ is equivalent to

$$
\begin{gathered}
a \alpha_{1} e^{0} \wedge e^{2}+\left(-(2 \mu-b) \alpha_{0}+\alpha_{2}\right) e^{1} \wedge e^{2}+\left(\alpha_{0}-b \alpha_{2}\right) e^{0} \wedge e^{1} \\
=s \alpha_{0} e^{1} \wedge e^{2}+s \alpha_{1} e^{0} \wedge e^{2}-s \alpha_{2} e^{0} \wedge e^{1} .
\end{gathered}
$$

This equation admits non-trivial solutions only if $\alpha_{2} \neq 0, b \neq s$ (which is required in order to have $\alpha_{0} \neq 0$ ) and

$$
b^{2}-2 b(s+\mu)+2(s \mu+1)=0 .
$$

The previous equation has the unique solution $b=\mu+s$, which satisfies $b \neq s$. Setting $b=\mu+s$ we obtain $\alpha_{0}=\mu \alpha_{2}$, whence $\alpha_{1}=0$.

- Case $\mathfrak{g}_{5}$. In the orthonormal frame for $\mathfrak{g}_{5}$ given in Appendix A, Equation $* \alpha=-s \mathrm{~d} \alpha$ is equivalent to

$$
\left(a \alpha_{1}+b \alpha_{2}\right) e^{0} \wedge e^{1}+\left(c \alpha_{1}+d \alpha_{2}\right) e^{0} \wedge e^{2}=s\left(\alpha_{0} e^{1} \wedge e^{2}+\alpha_{1} e^{0} \wedge e^{2}-\alpha_{2} e^{0} \wedge e^{1}\right) .
$$

This equation implies $\alpha_{0}=0$, which in turn yields $\alpha=0$. Therefore, $\mathfrak{g}_{5}$ does not admit null contact structures.

- Case $\mathfrak{g}_{6}$. In the orthonormal frame for $\mathfrak{g}_{6}$ given in Appendix A, Equation $* \alpha=-s \mathrm{~d} \alpha$ is equivalent to

$$
\left(d \alpha_{0}+c \alpha_{2}\right) e^{0} \wedge e^{1}+\left(-b \alpha_{0}-a \alpha_{2}\right) e^{1} \wedge e^{2}=s\left(\alpha_{0} e^{1} \wedge e^{2}+\alpha_{1} e^{0} \wedge e^{2}-\alpha_{2} e^{0} \wedge e^{1}\right) .
$$

We obtain $\alpha_{1}=0$, so that $\alpha_{0}=-\mu \alpha_{2}$ for $\mu \in \mathbb{Z}_{2}$. Consequently, $\mu a=s+b$ and $\mu d=s+c$. The remaining conditions follow from equations $a c=b d$ and $a+d \neq 0$, which must hold by Theorem A.1.

- Case $\mathfrak{g}_{7}$. In the orthonormal frame for $\mathfrak{g}_{7}$ given in Appendix A, Equation $* \alpha=-s \mathrm{~d} \alpha$ is equivalent to

$$
\begin{aligned}
\left(b \alpha_{0}+a \alpha_{1}+b \alpha_{2}\right) e^{0} \wedge e^{1} & +\left(d \alpha_{0}+c \alpha_{1}+d \alpha_{2}\right) e^{0} \wedge e^{2}+\left(b \alpha_{0}+a \alpha_{1}+b \alpha_{2}\right) e^{1} \wedge e^{2} \\
& =s\left(\alpha_{0} e^{1} \wedge e^{2}+\alpha_{1} e^{0} \wedge e^{2}-\alpha_{2} e^{0} \wedge e^{1}\right) .
\end{aligned}
$$

with $a+d \neq 0$ and $a c=0$. Imposing $a c=0$ implies $\alpha_{0}=0$, whence a Lorentzian Lie group with Lie algebra isomorphic to $\mathfrak{g}_{7}$ does not admit non-trivial null contact structures.

3.1. Sasakian null contact structures. The Sasakian condition of a Riemannian or Lorentzian (para) contact-metric structure with non-null Reeb vector field can be defined in terms of the integrability of certain endomorphism defined from $\phi$ on $T(M \times \mathbb{R})[3,9,17]$. In the null case $\varepsilon=0$, we proceed in analogous way and we introduce the following endomorphism, which mimics the definition occurring in the case $\varepsilon \neq 0$ : 


$$
J: T(M \times \mathbb{R}) \rightarrow T(M \times \mathbb{R}), \quad\left(v, c \partial_{q}\right) \mapsto\left(\phi(v)+c \xi, \alpha(v) \partial_{q}\right),
$$

where $q$ is the fixed canonical coordinate on $\mathbb{R}$ and $c \in \mathbb{R}$. A direct computation shows that $J^{2}=0$. We clarify first the relevant notion of integrability of a field of endomorphisms.

Definition 3.12. Let $E \in \Gamma\left(T N \otimes T^{*} N\right)$ be a field of endomorphisms on a manifold $N$. Then, $E$ is said to be integrable if around every point $n \in N$ there exists a coordinate system on which the local matrix representation of $E$ has constant coefficients.

For almost complex structures the previous definition is equivalent to the standard definition of integrability in terms of existence of holomorphic charts. Necessary and sufficient conditions for a nilpotent endomorphism (such as $J$ ) to be integrable have been studied in the literature, see $[13,36,39,50,52]$ and [12] for a thorough exposition of this and related topics. By a result of $\mathrm{G}$. Thomson [50, Theorem 2], we have that $J \in \Gamma(\operatorname{End}(T(M \times \mathbb{R})))$ is integrable if and only if the following three conditions hold simultaneously:

- The Nijenhuis torsion tensor of $J$ vanishes.

- $J$ is a zero-deformable field of endomorphisms.

- The distribution $\operatorname{Ker}(J) \subset T(M \times \mathbb{R})$ is involutive.

Recall that the Nijenhuis torsion tensor associated to $J$ is defined as

$$
\mathcal{N}_{J}\left(v_{1}, v_{2}\right)=\left[J\left(v_{1}\right), J\left(v_{2}\right)\right]-J\left[v_{1}, J\left(v_{2}\right)\right]-J\left[J\left(v_{1}\right), v_{2}\right]+J^{2}\left[v_{1}, v_{2}\right] .
$$

Note that since $J^{2}=0$, the last term in the right hand side identically vanishes. Also, we remind the reader that a certain field of endomorphisms is said to be zero-deformable if around every point of $M$ there exists a frame relative to which the Jordan form of this endomorphism field is constant.

Lemma 3.13. The endomorphism $J \in \Gamma(\operatorname{End}(T(M \times \mathbb{R})))$ is zero-deformable.

Proof. Fix a point in $M \times \mathbb{R}$ and consider the tangent-space basis $\left\{\xi, u, \phi(u), \partial_{q}\right\}$, where $\{\xi, u, \phi(u)\}$ is a light-cone basis. In this basis $J$ has the following matrix representation:

$$
J=\left[\begin{array}{cccc}
0 & 0 & -1 & 1 \\
0 & 0 & 0 & 0 \\
0 & 1 & 0 & 0 \\
0 & 1 & 0 & 0
\end{array}\right]
$$

Since the basis $\left(\xi, u, \phi(u), \partial_{q}\right)$ exists at every point in $M \times \mathbb{R}$, we conclude that $J$ is always locally conjugate to the same constant Jordan form.

Lemma 3.14. The distribution $\operatorname{Ker}(J) \subset T(M \times \mathbb{R})$ is involutive.

Proof. Fix a local frame $\left\{\xi, u, \phi(u), \partial_{q}\right\}$ on $M \times \mathbb{R}$, where $\{\xi, u, \phi(u)\}$ is a light-cone frame for $M$. The kernel of $J$ is locally spanned by

$$
\operatorname{Ker}(J)=\operatorname{Span}_{C^{\infty}}\left(\xi, \phi(u)+\partial_{q}\right) .
$$

Lemma 3.7 implies now:

$$
\left[\xi, \phi(u)+\partial_{q}\right]=[\xi, \phi(u)]=(\mu-c) \xi,
$$

and we conclude.

Proposition 3.15. A null contact metric structure $(g, \alpha)$ is Sasakian if and only if its associated endomorphism $J: T(M \times \mathbb{R}) \rightarrow T(M \times \mathbb{R})$ is integrable. 
Proof. Assume first that $(g, \alpha)$ is Sasakian, that is, $\mathfrak{h}=0$. By Lemma 3.13 the endomorphism $J$ is zero-deformable and by Lemma 3.14 its kernel $\operatorname{Ker}(J)$ is involutive. Therefore by Theorem $[50$, Theorem 2] we only need to show that $\mathcal{N}_{J}$ vanishes to prove that $J$ is integrable. Since $\mathcal{N}_{J}$ is a tensor, it is enough to prove that it vanishes on a light cone frame $\left\{\xi, u, \phi(u), \partial_{q}\right\}$. We compute:

$$
\begin{gathered}
\mathcal{N}_{J}(\xi, u)=-J[\xi, \phi(u)]=0, \quad \mathcal{N}_{J}(\xi, \phi(u))=-J[\xi, J(\phi(u))]=0, \\
\mathcal{N}_{J}(u, \phi(u))=-J[J(\phi(u)), J(\phi(u))]=0, \quad \mathcal{N}_{J}\left(\xi, \partial_{q}\right)=-J\left[\xi, J\left(\partial_{q}\right)\right]=0, \\
\mathcal{N}_{J}\left(u, \partial_{q}\right)=[\phi(u), \xi]-J[u, \xi]=-\mathcal{L}_{\xi}(\phi(u))+J\left(\mathcal{L}_{\xi} u\right)=-\mathcal{L}_{\xi}(\phi(u))+\phi\left(\mathcal{L}_{\xi} u\right)=-\mathfrak{h}(u)=0, \\
\mathcal{N}_{J}\left(\phi(u), \partial_{q}\right)=\left[J(\phi(u)), J\left(\partial_{q}\right)\right]-J\left[\phi(u), J\left(\partial_{q}\right)\right]=\left[\phi^{2}(u), \xi\right]-J[\phi(u), \xi]=\phi^{2}\left(\mathcal{L}_{\xi} u\right)=0,
\end{gathered}
$$

whence $J$ is integrable. The converse follows now directly by applying the previous formulae, upon use of Lemmas 3.13 and 3.14 and the fact that $\mathfrak{h}(u)=0$ if and only if $\mathfrak{h}=0$.

We proceed now to classify all left-invariant Sasakian null contact structures on simply-connected, Lorenztian, three-dimensional Lie groups by exploiting Proposition 3.11.

Proposition 3.16. A three-dimensional connected and simply connected Lie group $\mathrm{G}$ admits a left-invariant Sasakian null contact structure $(g, \alpha)$ if and only if $(\mathrm{G}, g, \alpha)$ is isomorphic, through a possibly orientation-reversing isometry, to one of the items listed in the following table in terms of the orthonormal frame $\left\{e_{0}, e_{1}, e_{2}\right\}$ appearing in Theorem A.1:

\begin{tabular}{|c|c|c|c|}
\hline $\mathfrak{g}$ & Structure constants $\left(s \in \mathbb{Z}_{2}\right)$ & $\alpha$ & $\mathrm{G}$ \\
\hline $\mathfrak{g}_{1}$ & $a \neq 0, b=s$ & $\alpha_{1}=0, \alpha_{0}=-\alpha_{2}$ & $\widetilde{\mathrm{Sl}}(2, \mathbb{R})$ \\
\hline $\mathfrak{g}_{3}$ & $a=b=c=s$ & $\alpha_{0}^{2}=\alpha_{1}^{2}+\alpha_{2}^{2}$ & $\widetilde{\mathrm{Sl}}(2, \mathbb{R})$ \\
\hline $\mathfrak{g}_{4}$ & $b=s+\mu, a=s, \mu \in \mathbb{Z}_{2}$ & $\alpha_{1}=0, \alpha_{0}=\mu \alpha_{2}$ & $\widetilde{\mathrm{Sl}}(2, \mathbb{R})$ \\
\hline $\mathfrak{g}_{6}$ & $b=c=-\frac{s}{2}, a=d=\frac{\mu s}{2}$, & $\alpha_{1}=0, \alpha_{0}=-\mu \alpha_{2}$ & $\mathfrak{G}_{6}$ \\
\hline
\end{tabular}

Proof. We proceed by verifying which cases in Proposition 3.11 satisfy $\mathfrak{h}=0$. By Proposition 3.4 , it is enough to prove that $\mathfrak{h}(u)=0$, where $u \in\{\xi, u, \phi(u)\}$ belongs to a light-cone frame. In the following computations it will be very convenient to use that the matrix expression of the characteristic endomorphism $\phi$ in the orthonormal basis $\left\{e_{0}, e_{1}, e_{2}\right\}$ used at Theorem A.1 is given by

$$
\phi=\left[\begin{array}{ccc}
0 & \alpha_{2} & -\alpha_{1} \\
\alpha_{2} & 0 & \alpha_{0} \\
-\alpha_{1} & -\alpha_{0} & 0
\end{array}\right] .
$$

Furthermore, the Reeb vector field $\xi$ is given by $\xi=-\alpha_{0} e_{0}+\alpha_{1} e_{1}+\alpha_{2} e_{2}$.

- Case $\mathfrak{g}_{1}$. Since $\alpha_{1}=0$ by Proposition 3.11, then the Reeb vector field reads $\xi=-\alpha_{0}\left(e_{0}+e_{2}\right)$. A direct computation shows that $\left(\xi, u=\frac{1}{2 \alpha_{0}}\left(e_{0}-e_{2}\right),-e_{1}\right)$ is a light-cone frame (where $\left.\phi(u)=-e_{1}\right)$. Hence

$$
\mathfrak{h}(u)=\alpha_{0}\left[e_{0}, e_{1}\right]+\alpha_{0}\left[e_{2}, e_{1}\right]+\phi\left(\left[e_{2}, e_{0}\right]\right)=0,
$$

implying that every null contact structure on $\mathfrak{g}_{1}$ is Sasakian.

- Case $\mathfrak{g}_{3}$. According to Proposition 3.11 we distinguish between the cases $\alpha_{1}=0$ with $\alpha_{2} \neq 0$, $\alpha_{2}=0$ with $\alpha_{1} \neq 0$ and $\alpha_{1}, \alpha_{2} \neq 0$. If $\alpha_{1}=0$, then $\xi=\alpha_{0}\left(-e_{0}+\mu e_{2}\right)$ and $\left(\xi, u=\frac{1}{2 \alpha_{0}}\left(e_{0}+\right.\right.$ $\left.\left.\mu e_{2}\right), \mu e_{1}\right)$ is a light-cone frame. We obtain, after using that $b=c=s$ :

$$
\mathfrak{h}(u)=\alpha_{0}(s-a) e_{0}+\mu \alpha_{0}(a-s) e_{2} .
$$


Hence, $\mathfrak{h}=0$ if and only if $a=s$. Similarly, if $\alpha_{2}=0$, then $\xi=\alpha_{0}\left(-e_{0}+\mu e_{1}\right)$ and $\{\xi, u=$ $\left.\frac{1}{2 \alpha_{0}}\left(e_{0}+\mu e_{1}\right), \mu e_{2}\right\}$ is a light-cone frame (with $\left.\phi(u)=\mu e_{2}\right)$. Since $a=c=s$, we compute:

$$
\mathfrak{h}(u)=\alpha_{0}(s-b) e_{0}+\mu \alpha_{0}(b-s) e_{1} .
$$

Hence $\mathfrak{h}=0$ if and only if $b=s$. The case $\alpha_{1}, \alpha_{2} \neq 0$ follows along similar lines.

- Case $\mathfrak{g}_{4}$. In this case we have $\xi=\alpha_{0}\left(-e_{0}+\mu e_{2}\right)$ and get a light-cone frame $\left(\xi, u=\frac{1}{2 \alpha_{0}}\left(e_{0}+\right.\right.$ $\left.\left.\mu e_{2}\right), \mu e_{1}\right)$. We obtain:

$$
\mathfrak{h}(u)=\alpha_{0}(-(2 \mu-b)+\mu-a) e_{0}+\alpha_{0}(1-\mu b+\mu a) e_{2},
$$

which vanishes if and only if

$$
1-\mu b+\mu a=0 .
$$

This is in turn equivalent to the constraint $a=s$.

- Case $\mathfrak{g}_{6}$. In this case we have $\xi=-\alpha_{0}\left(e_{0}+\mu e_{2}\right)$ and $\left\{\xi, u=\frac{1}{2 \alpha_{0}}\left(e_{0}-\mu e_{2}\right),-\mu e_{1}\right\}$ is a light-cone frame, where $\phi(u)=-\mu e_{1}$. We find:

$$
\mathfrak{h}(u)=-\alpha_{0} \mu(d+\mu b) e_{0}-\alpha_{0} \mu(c+\mu a) e_{2},
$$

whence $c=-\mu a$ and $d=-\mu b$. Taking into account now the constraints stated in Proposition 3.11 we conclude.

3.2. Null K-contact structures. For three-dimensional Riemannian contact structures, Lorentzian contact structures and para-contact structures, the K-contact and Sasakian conditions are equivalent. However, this fails to be the case for null contact structures, as the following Example shows.

Example 3.17. Take $M$ to be a connected and simply connected Lie group admitting a left-invariant global co-frame $\left\{e^{+}, e^{-}, e^{2}\right\}$ satisfying:

$$
\mathrm{d} e^{+}=-a e^{+} \wedge e^{-}-e^{+} \wedge e^{2}, \quad \mathrm{~d} e^{-}=e^{-} \wedge e^{2}, \quad \mathrm{~d} e^{2}=e^{+} \wedge e^{-}-a e^{-} \wedge e^{2},
$$

where $a \in \mathbb{R} \backslash\{0\}$. We denote by $\left\{e_{+}, e_{-}, e_{2}\right\}$ the frame dual to $\left\{e^{+}, e^{-}, e^{2}\right\}$. We equip $M$ with the Lorentzian metric

$$
g=e^{+} \odot e^{-}+e^{2} \otimes e^{2}
$$

and we fix the volume form to be $\nu=e^{-} \wedge e^{+} \wedge e^{2}$. Set $\alpha \stackrel{\text { def. }}{=} e^{-}$, whence $\xi=e_{+}$. Then, $(g, \alpha)$ defines a null contact structure on $M$ since $g(\xi, \xi)=0$ and

$$
* \alpha=-\alpha \wedge e^{2}=-\mathrm{d} \alpha .
$$

The characteristic endomorphism $\phi$ is given by:

$$
\phi(\xi)=0, \quad \phi\left(e_{-}\right)=\left(\iota_{e_{-}} * \alpha\right)^{\sharp}=-e_{2}, \quad \phi\left(e_{2}\right)=\left(\iota_{e_{2}} * \alpha\right)^{\sharp}=\xi .
$$

Therefore, $\left\{\xi, e_{-},-e_{2}\right\}$ yields a light-cone frame. Similarly, $\phi^{2}$ can be shown to satisfy:

$$
\phi^{2}(\xi)=0, \quad \phi^{2}\left(e_{-}\right)=-\phi\left(e_{2}\right)=-\xi, \quad \phi^{2}\left(e_{2}\right)=0 .
$$

Hence we obtain $\phi^{2}=-\xi \otimes \alpha$ and $\phi^{3}=0$, as required. The fact that $(g, \alpha)$ is Sasakian follows now from the following computation:

$$
\mathfrak{h}\left(e_{-}\right)=\left[\xi, \phi\left(e_{-}\right)\right]-\phi\left(\left[\xi, e_{-}\right]\right)=-\xi+\xi=0 .
$$


which, together with Proposition 3.4 implies that $\mathfrak{h}$ vanishes identically. On the other hand, $\xi$ cannot be a Killing vector field, because:

$$
\left(\mathcal{L}_{\xi} g\right)\left(e_{-}, e_{-}\right)=-2 g\left(\left[\xi, e_{-}\right], e_{-}\right)=-2 a g\left(\xi, e_{-}\right)=-2 a \neq 0
$$

and $a \neq 0$ by assumption. Therefore $(g, \alpha)$ is a Sasakian null contact structure which fails to be K-contact.

Hence, the Sasakian condition does not imply the K-contact condition.

Proposition 3.18. Every three-dimensional null K-contact structure $(g, \alpha)$ is Sasakian.

Proof. Let $\xi$ denote the Reeb vector field associated to $(g, \alpha)$ and choose a light-cone frame $\{\xi, u, \phi(u)\}$. From the K-contact condition we have:

$$
0=-\left(\mathcal{L}_{\xi} g\right)(u, \phi(u))=g\left(\mathcal{L}_{\xi} u, \phi(u)\right)+g\left(u, \phi\left(\mathcal{L}_{\xi} u\right)\right)+g(u, \mathfrak{h}(u))=g(u, \mathfrak{h}(u)),
$$

where we have used that $\phi$ is skew-adjoint with respect to $g$. On the other hand, Corollary 3.4 implies that $\mathfrak{h}(u)=\mu \xi$ for some function $\mu \in C^{\infty}(M)$. Since $g(u, \xi)=1$, equation $g(u, \mathfrak{h}(u))=0$ is equivalent to $\mu=0$ and thus $\mathfrak{h}=0$.

Proposition 3.19. Let $\xi$ denote the Reeb vector field associated to a Sasakian null-contact structure $(g, \alpha)$, and let $\{\xi, u, \phi(u)\}$ be a light-cone frame. Then, $(g, \alpha)$ is K-contact if and only if:

$$
g\left(\mathcal{L}_{\xi} u, u\right)=0,
$$

Proof. We evaluate $\mathcal{L}_{\xi} g$ on a light-cone frame $\{\xi, u, \phi(u)\}$. A direct computation using Lemma 3.7 shows that the only non-trivial term is

$$
\left(\mathcal{L}_{\xi} g\right)(u, u)=-2 g\left(\mathcal{L}_{\xi} u, u\right)
$$

and we conclude.

Remark 3.20. Indeed, not every Sasakian null contact structure satisfies that $g\left(\mathcal{L}_{\xi} u, u\right)=0$. For instance, in Example 3.17, we have that:

$$
g\left(\mathcal{L}_{\xi} u, u\right)=g\left(\left[e_{+}, e_{-}\right], e_{-}\right)=a \neq 0,
$$

as expected. Therefore we learn that the Sasakian condition for null contact structures is weaker than the K-contact condition. Interestingly enough, this is in sharp contrast to what occurs for non-null contact structures: for such structures these conditions are equivalent in three dimensions whereas in higher dimensions the Sasakian condition is stronger than the K-contact condition $[9,15]$.

Using the classification of simply connected, Lorentzian, three-dimensional Lie groups admitting left-invariant Sasakian null contact structures presented in Proposition 3.16, we obtain in the following an analogous classification for null K-contact structures.

Proposition 3.21. A three-dimensional connected and simply connected Lie group $\mathrm{G}$ admits a left-invariant null $K$-contact contact structure $(g, \alpha)$ if and only if $(\mathrm{G}, g, \alpha)$ is isomorphic, through a possibly orientation-reversing isometry, to one of the items listed in the following table in terms of the orthonormal frame $\left\{e_{0}, e_{1}, e_{2}\right\}$ appearing in Theorem A.1: 


\begin{tabular}{|c|c|c|c|}
\hline $\mathfrak{g}$ & Structure constants $\left(s \in \mathbb{Z}_{2}\right)$ & $\alpha$ & $\mathrm{G}$ \\
\hline $\mathfrak{g}_{3}$ & $a=b=c=s$ & $\alpha_{0}^{2}=\alpha_{1}^{2}+\alpha_{2}^{2}$ & $\widetilde{\mathrm{Sl}}(2, \mathbb{R})$ \\
\hline $\mathfrak{g}_{4}$ & $b=s+\mu, a=s, \mu \in \mathbb{Z}_{2}$ & $\alpha_{1}=0, \alpha_{0}=\mu \alpha_{2}$ & $\widetilde{\mathrm{Sl}}(2, \mathbb{R})$ \\
\hline $\mathfrak{g}_{6}$ & $b=c=-\frac{s}{2}, a=d=\frac{\mu s}{2}$ & $\alpha_{1}=0, \alpha_{0}=-\mu \alpha_{2}$ & $\mathfrak{G}_{6}$ \\
\hline
\end{tabular}

Proof. Proposition 3.18 states that every null K-contact structure is Sasakian. Therefore, we proceed by checking which cases in Proposition 3.16 are in fact K-contact. By Proposition 3.19, we will test K-contactness by verifying if $g([\xi, u], u)=0$. We use the terminology introduced in Propositions 3.16 and 3.18:

- Case $\mathfrak{g}_{1}$. We obtain $[\xi, u]=\left[e_{0}, e_{2}\right]=-s e_{1}-a e_{2}-a e_{0} \neq 0$, where $a \neq 0$ by Theorem A.1. Hence:

$$
g([\xi, u], u)=\frac{a}{\alpha_{0}} \neq 0
$$

whence $\mathfrak{g}_{1}$ does not admit left-invariant null K-contact structures. In fact, Example 3.17 corresponds to a Lie group of type $\mathfrak{g}_{1}$.

- Case $\mathfrak{g}_{3}$. We distinguish between the cases $\alpha_{1}=0$ or $\alpha_{2}=0$ and $\alpha_{1}, \alpha_{2} \neq 0$. If $\alpha_{1}=0$, then $[\xi, u]=\mu\left[e_{2}, e_{0}\right]=\mu e_{1}=\phi(u)$. Hence $g([\xi, u], u)=0$. Similarly, if $\alpha_{2}=0$, then $[\xi, u]=$ $\mu\left[e_{1}, e_{0}\right]=-\mu e_{2}=\phi(u)$ and $g([\xi, u], u)=0$. For $\alpha_{1}, \alpha_{2} \neq 0$ a direct computation shows that $\left(\mathcal{L}_{\xi} g\right)\left(e_{i}, e_{j}\right)=0$ for every $i, j \in\{1,2,3\}$, whence $\mathcal{L}_{\xi} g=0$ and every left-invariant Sasakian null contact structure on $\mathfrak{g}_{3}$ is also K-contact.

- Case $\mathfrak{g}_{4}$. We have $[\xi, u]=\mu\left[e_{2}, e_{0}\right]=\mu a e_{1}=a \phi(u)$, so $g([\xi, u], u)=0$ and the Sasakian and K-contact conditions are equivalent on $\mathfrak{g}_{4}$.

- Case $\mathfrak{g}_{6}$. We have $[\xi, u]=-\mu\left[e_{2}, e_{0}\right]=0$, so $g([\xi, u], u)=0$. Hence the Sasakian and K-contact conditions are equivalent on $\mathfrak{g}_{6}$.

The previous Proposition and Example 3.17 show that the theory of null K-contact structures and Sasakian null contact structures in three dimensions has the potential to be richer than its $\varepsilon \neq 0$ counterpart, where the Sasakian and K-contact conditions are equivalent. Further investigation of this issue is beyond the scope of this manuscript.

\section{4. $\varepsilon \eta$-EINSTEIN $\varepsilon$-CONTACT METRIC MANIFOLDS}

We introduce in this section the notion of $\varepsilon \eta$-Einstein $\varepsilon$-contact metric structure on an oriented three-manifold $N$, which is a particular case of the standard notion of $\eta$-Einstein Riemannian/Lorentzian contact metric structure when the Reeb vector field has non-vanishing norm. The definition is motivated by the structure of six-dimensional supergravity (coupled to a tensor multiplet) and its solutions, see Section 5 and Theorem 5.4 for details and applications.

Definition 4.1. An $\varepsilon$-contact metric structure $(g, \alpha, \varepsilon)$ on $N$ is said to be $\varepsilon \eta$-Einstein if the Ricci curvature tensor $\operatorname{Ric}^{g}$ of $g$ satisfies

$$
\operatorname{Ric}^{g}=\frac{\mathrm{s}_{g}}{2}\left(\lambda^{2}+\kappa \varepsilon\right) g-\mathrm{s}_{g} \kappa \alpha \otimes \alpha,
$$

where $\mathrm{s}_{g}=1$ if $g$ is Riemannian, $\mathrm{s}_{g}=-1$ if $g$ is Lorentzian and $\lambda, \kappa \in \mathbb{R}$ are real constants such that $\kappa \geq 0$ if $\mathrm{s}_{g}=-1$. 
Remark 4.2. Whenever there is no possible confusion, we may abbreviate notation and denote an $\varepsilon \eta$-Einstein contact metric structure $(g, \alpha, \varepsilon)$ just by $\varepsilon \eta$-Einstein contact structure.

Remark 4.3. Recall that we denote by $\mathrm{Q}^{g} \in \Gamma\left(T M \otimes T^{*} M\right)$ the endomorphism associated to $\mathrm{Ric}^{g}$. Then, $(g, \alpha, \varepsilon)$ is $\varepsilon \eta$-Einstein if and only if

$$
\mathrm{Q}^{g}=\frac{\mathrm{s}_{g}}{2}\left(\lambda^{2}+\kappa \varepsilon\right) \mathrm{Id}-\mathrm{s}_{g} \kappa \xi \otimes \alpha,
$$

in which case

$$
\mathrm{Q}^{g}(\xi)=\frac{\mathrm{s}_{g}}{2}\left(\lambda^{2}-\kappa \varepsilon\right) \xi
$$

Therefore $\xi$ is an eigenvector of $\mathrm{Q}^{g}$ with eigenvalue $\rho_{\xi}=\frac{\mathrm{s}_{g}}{2}\left(\lambda^{2}-\kappa \varepsilon\right)$.

Definition 4.4. We denote by $\mathrm{PCont}^{\varepsilon \eta}\left(\varepsilon, \lambda^{2}, \kappa\right)$ the category of $\varepsilon \eta$-Einstein $\varepsilon$-contact structures with respect to $\lambda^{2}$ and $\kappa$ and whose Reeb vector field is of norm $\varepsilon$. Likewise, denote by PCont $_{L}^{\varepsilon \eta}\left(\varepsilon, \lambda^{2}, \kappa\right)$ (PCont $\left.{ }_{R}^{\varepsilon \eta}\left(\lambda^{2}, \kappa\right)\right)$ the category of Lorentzian (Riemannian) $\varepsilon \eta$-Einstein $\varepsilon$-contact structures with respect to $\lambda^{2}, \kappa$ and whose Reeb vector field is of norm $\varepsilon$.

Remark 4.5. Let $(g, \alpha, \varepsilon) \in \operatorname{PCont}^{\varepsilon \eta}\left(\varepsilon, \lambda^{2}, \kappa\right)$ with $\varepsilon \mathfrak{s}_{g}=1$. By Definition 8, we have:

$$
\operatorname{Ric}^{g}(\xi, \xi)=\frac{\mathfrak{s}_{g}}{2}\left(\lambda^{2} \varepsilon-\kappa\right) .
$$

On the other hand, by Proposition $2.21(g, \alpha, \varepsilon)$ is Sasakian if and only if $\operatorname{Ric}^{g}(\xi, \xi)=\mathfrak{s}_{g} \frac{\varepsilon}{2}$. This is equivalent to

$$
\lambda^{2}=1+\kappa \varepsilon,
$$

In other words, an $\varepsilon \eta$-Einstein $\varepsilon$-contact structure $(g, \alpha, \varepsilon)$ such that $\mathfrak{s}_{g} \varepsilon=1$ is Sasakian if and only if $(g, \alpha, \varepsilon) \in \operatorname{PCont}^{\varepsilon \eta}(\varepsilon, 1+\varepsilon \kappa, \kappa)$.

Lemma 4.6. Let $(N, g, \alpha, \varepsilon) \in \mathrm{PCont}^{\varepsilon \eta}\left(\varepsilon, \lambda^{2}, \kappa\right)$. Then, the following equation holds:

$$
\mathrm{R}^{g}\left(v_{1}, v_{2}\right)(\xi)=\mathcal{K}\left(\alpha\left(v_{2}\right) v_{1}-\alpha\left(v_{1}\right) v_{2}\right),
$$

where

$$
\mathcal{K} \stackrel{\text { def. }}{=} \frac{\mathfrak{s}_{g}\left(\lambda^{2}-\varepsilon \kappa\right)}{4}
$$

and $\mathrm{R}^{g}$ denotes the Riemann tensor on $(N, g)$.

Proof. The results follows directly by plugging the $\varepsilon \eta$-Einstein condition in the expression for the Riemann tensor of a (pseudo-)Riemannian three-manifold in terms of its Ricci curvature.

Remark 4.7. The previous Proposition implies that a Riemannian $\varepsilon \eta$-Einstein contact manifold $(M, g, \alpha, \varepsilon) \in \mathrm{PCont}_{R}^{\varepsilon \eta}\left(\lambda^{2}, \kappa\right)$ is a $(\kappa, \mu)$ manifold or, alternatively, a Riemannian contact threemanifold whose Reeb vector field belongs to the nullity distribution [11].

We consider now the $\varepsilon \eta$-Einstein condition on globally hyperbolic Lorentzian three-manifolds, making contact with Section 2.1, where the $\varepsilon$-contact condition for a one-form on a globally hyperbolic Lorentzian three-manifold was considered. Therefore, using the notation of Section 2.1, we set:

$$
(N, g)=\left(\mathbb{R} \times \mathrm{X}_{t}, g=-\beta_{t}^{2} \mathrm{~d} t \otimes \mathrm{d} t+q_{t}\right), \quad \alpha=F_{t} e^{0}+\alpha_{t}^{\perp},
$$

where $\left\{\beta_{t}\right\}_{t \in \mathbb{R}},\left\{F_{t}\right\}_{t \in \mathbb{R}},\left\{\alpha_{t}\right\}_{t \in \mathbb{R}}$ and $\left\{q_{t}\right\}_{t \in \mathbb{R}}$ are parametric families on $\mathrm{X}_{t}, e^{0}=\beta_{t} \mathrm{~d} t$ is the normalized time-like one-form induced by the globally hyperbolic presentation of $(N, g)$ and where we have considered, for every $t \in \mathbb{R}$,

$$
\mathrm{X}_{t} \stackrel{\text { def. }}{=}\{t\} \times \mathrm{X} \subset N
$$


as an embedded manifold. We introduce the familiar Weingarten tensor $W_{t}$ and second fundamental form $\Theta_{t}$ :

$$
W_{t}=-\left.\nabla n_{t}\right|_{T \mathrm{X}_{t}} \in \Omega^{1}\left(T \mathrm{X}_{t}\right), \quad \Theta_{t}=-\left.\frac{1}{2 \beta_{t}} \mathcal{L}_{\partial_{t}} g\right|_{T \mathrm{X}_{t} \times T \mathrm{X}_{t}} \in \Gamma\left(T^{*} \mathrm{X}_{t} \odot T^{*} \mathrm{X}_{t}\right)
$$

associated to the embedding $X_{t} \subset N$. The trace of $\Theta_{t}$ with respect to $q_{t}$, which we denote by $\operatorname{Tr}_{q_{t}}\left(\Theta_{t}\right)$, is the mean curvature of the embedded surface $X_{t} \subset N$.

Proposition 4.8. A tuple $\left(\left\{\beta_{t}\right\}_{t \in \mathbb{R}},\left\{F_{t}\right\}_{t \in \mathbb{R}},\left\{\alpha_{t}^{\perp}\right\}_{t \in \mathbb{R}},\left\{q_{t}\right\}_{t \in \mathbb{R}}\right)$ on $\mathrm{X}_{t}$ defines an $\varepsilon \eta$-Einstein $\varepsilon$ contact structure as prescribed in (9) if and only if:

$$
\begin{gathered}
\mathrm{d}_{\mathrm{X}_{t}} \alpha_{t}^{\perp}=F_{t} \nu_{q_{t}}, \quad{ }_{q_{t}} \alpha_{t}^{\perp}+\frac{1}{\beta_{t}} \mathrm{~d}_{\mathrm{X}}\left(\beta_{t} F_{t}\right)=\mathcal{L}_{n_{t}} \alpha_{t}^{\perp}, \quad\left|\alpha_{t}^{\perp}\right|_{q_{t}}^{2}=\varepsilon+F_{t}^{2}, \\
\mathrm{R}^{q_{t}}-\left|\Theta_{t}\right|_{q_{t}}^{2}+\left(\operatorname{Tr}_{q_{t}} \Theta\right)^{2}+\mathfrak{c}=2 \kappa F_{t}^{2}, \quad \mathrm{~d}_{\mathrm{x}_{t}} \operatorname{Tr}_{q_{t}}\left(\Theta_{t}\right)+\operatorname{div}_{q_{t}}\left(\Theta_{t}\right)=\kappa F_{t} \alpha_{t}^{\perp} \\
\operatorname{Ric}^{q_{t}}+\operatorname{Tr}_{q_{t}}\left(\Theta_{t}\right) \Theta_{t}-2 \Theta_{t}\left(\mathrm{Id} \otimes W_{t}\right)-\frac{1}{\beta_{t}}\left(\dot{\Theta}_{t}+\nabla^{q_{t}} \mathrm{~d}_{\mathrm{x}_{t}} \beta_{t}\right)=\kappa \alpha_{t}^{\perp} \otimes \alpha_{t}^{\perp}-\frac{1}{2}\left(\lambda^{2}+\kappa \varepsilon\right) q_{t},
\end{gathered}
$$

where $\mathcal{L}$ denotes Lie derivative, $\mathrm{d}_{\mathrm{x}_{t}}$ denotes the exterior derivative on $\mathrm{X}_{t}, \operatorname{Tr}_{q_{t}}$ denotes trace with respect to $q_{t}, \nabla^{q_{t}}$ represents the Levi-Civita connection of $q_{t}$ and $\mathfrak{c}=\frac{1}{2}\left(5 \lambda^{2}+3 \kappa \varepsilon\right)$ is a constant.

Proof. The first line of equations in (10) is proven in Proposition 2.22. A direct computation shows that:

$$
\begin{gathered}
\operatorname{Ric}^{g}(n, n)=\left|\Theta_{t}\right|_{q_{t}}^{2}+\frac{1}{\beta_{t}}\left(\left(\operatorname{tr}_{q_{t}} \dot{\Theta}\right)^{2}+\Delta_{q_{t}} \beta_{t}\right),\left.\quad \operatorname{Ric}^{g}(n)\right|_{T \mathrm{X}_{t}}=\mathrm{d}_{\mathrm{X}_{t}} \operatorname{Tr}_{q_{t}}\left(\Theta_{t}\right)+\operatorname{div}_{q_{t}}\left(\Theta_{t}\right), \\
\left.\operatorname{Ric}^{g}\right|_{T \mathrm{X}_{t} \otimes T \mathrm{X}_{t}}=\operatorname{Ric}^{q_{t}}+\operatorname{Tr}\left(\Theta_{t}\right) \Theta_{t}-2 \Theta_{t}\left(\mathrm{Id} \otimes W_{t}\right)-\frac{1}{\beta_{t}}\left(\dot{\Theta}_{t}+\nabla^{q_{t}} \mathrm{~d}_{\mathrm{X}_{t}} \beta_{t}\right),
\end{gathered}
$$

Plugging these equations in the $\varepsilon \eta$-Einstein condition (8) and combining them with the trace of (8) we obtain the second and third lines in (10). For more details the reader is referred to [5] and references therein.

Therefore, the $\varepsilon \eta$-Einstein condition on a globally hyperbolic Lorentian three manifold becomes, as expected, a dynamical equation on the evolution of a pair of functions, a one-form and a metric on a two-dimensional oriented manifold. Note that the only equations in (10) that contain time derivatives correspond to the second equation in the first line and the equation in the third line. These become the evolution equations for the tuple $\left(\left\{\beta_{t}\right\}_{t \in \mathbb{R}},\left\{F_{t}\right\}_{t \in \mathbb{R}},\left\{\alpha_{t}^{\perp}\right\}_{t \in \mathbb{R}},\left\{q_{t}\right\}_{t \in \mathbb{R}}\right)$, while the rest of equations can be considered as constraint equations in order to formulate a Cauchy or initial value problem for Lorentzian $\varepsilon \eta$-Einstein $\varepsilon$-contact structures. Set:

$$
\mathrm{X} \stackrel{\text { def. }}{=} \mathrm{X}_{0}, \quad \Theta \stackrel{\text { def. }}{=} \Theta_{0}, \quad q \stackrel{\text { def. }}{=} q_{0}, \quad \alpha \stackrel{\perp \text { def. }}{=} \alpha_{0}^{\perp}, \quad \beta \stackrel{\text { def. }}{=} \beta_{0}, \quad F \stackrel{\text { def. }}{=} F_{0},
$$

and consider $\mathrm{X}$ as the Cauchy surface for the zero-time intial values $\left(q, \Theta, F, \alpha^{\perp}\right)$ associated to a given tuple

$$
\left(\left\{\beta_{t}\right\}_{t \in \mathbb{R}},\left\{F_{t}\right\}_{t \in \mathbb{R}},\left\{\alpha_{t}^{\perp}\right\}_{t \in \mathbb{R}},\left\{q_{t}\right\}_{t \in \mathbb{R}}\right)
$$

satisfying equations (10). The following result is a direct consequence Proposition 4.8.

Proposition 4.9. If $\left(\left\{\beta_{t}\right\}_{t \in \mathbb{R}},\left\{F_{t}\right\}_{t \in \mathbb{R}},\left\{\alpha_{t}^{\perp}\right\}_{t \in \mathbb{R}},\left\{q_{t}\right\}_{t \in \mathbb{R}}\right)$ is a solution of (10) then $\left(q, \Theta, F, \alpha^{\perp}\right)$ satisfies:

$$
\begin{gathered}
\mathrm{d}_{\mathrm{X}} \alpha^{\perp}=F \nu_{q}, \quad\left|\alpha^{\perp}\right|_{q}^{2}=\varepsilon+F^{2}, \\
\mathrm{R}^{q}-|\Theta|_{q}^{2}+\left(\operatorname{Tr}_{q} \Theta\right)^{2}+\mathfrak{c}=2 \kappa F^{2}, \quad \mathrm{dX}_{\mathrm{X}} \operatorname{Tr}_{q}(\Theta)+\operatorname{div}_{q}(\Theta)=\kappa F \alpha^{\perp},
\end{gathered}
$$

where $\Theta$ is a symmetric bilinear form on $\mathrm{X}$. 
Remark 4.10. Equations (11), to which we will refer as the constraint equations of an $\varepsilon \eta$-Einstein structure, generalize the well-known constraint equations of General Relativity coupled to a cosmological constant in $(2+1)$ dimensions via the coupling of an $\varepsilon$-contact structure. If we take $F=0$ then, the second equation in the first line of (11) forces $\varepsilon=1$ and the whole system decouples. The second line in (11) corresponds in this case to the constraint equations of General Relativity coupled to a cosmological constant. This system has been studied in the literature, see $[4,45]$ and references therein. In fact, it would be interesting to explore if a Hamiltonian formulation on (the cotangent space of) Teichmüller space, in the lines of the one presented in [4, 45], can be developed also for $\varepsilon \eta$-Einstein structures. Note that, under the assumption $F=0$ the first line reduces to the condition of $\alpha^{\perp}$ being closed and of constant norm, a condition which if $\mathrm{X}$ is compact can only be satisfied on the torus.

Proving the converse of Proposition 4.9, that is, proving that for every solution of (11) there exists a tuple $\left(\left\{\beta_{t}\right\}_{t \in \mathbb{R}},\left\{F_{t}\right\}_{t \in \mathbb{R}},\left\{\alpha_{t}^{\perp}\right\}_{t \in \mathbb{R}},\left\{q_{t}\right\}_{t \in \mathbb{R}}\right)$ fulfilling (10), would solve the initial value problem of $\varepsilon \eta$-Einstein $\varepsilon$-contact structures. Addressing this problem is beyond the scope of this manuscript and will be considered elsewhere. Having said this, we expect the converse to hold due to the fact that $\varepsilon \eta$-Einstein $\varepsilon$-contact condition arises in a supergravity theory, which is expected to pose consistent initial value problems due to their supersymmetric structure [23].

We distinguish the cases $\kappa=0$ and $\kappa \neq 0$. Due to its complexity, we plan to investigate the case $\kappa \neq 0$ in a separate publication, so we focus instead on the case $\kappa=0$ in the following, with the goal of showing existence of particular solutions. If $\kappa=0$ then Equations (11) decouple again (see Remark 4.10) and the second line in (11) corresponds to the constraint equations of General Relativity coupled to a cosmological constant. Hence, we focus on the first line in (11), which we rewrite as follows:

$$
* \mathrm{~d}_{X} \alpha^{\perp}+\sqrt{\left|\alpha^{\perp}\right|_{q}^{2}-\varepsilon}=0
$$

with variable given by a one-form $\alpha^{\perp} \in \Omega^{1}(\mathrm{X})$ on a complete Riemann surface (X, $q$ ). We focus on the null-contact case $\varepsilon=0$, since it seems to be new in the literature. In this case, the previous equation reduces simply to

$$
* \mathrm{~d}_{X} \alpha^{\perp}+\left|\alpha^{\perp}\right|_{q}=0 .
$$

We introduce now local isothermal coordinates $(x, y)$ on $\mathrm{X}$, in which the metric $q$ reads $q=F^{2}\left(\mathrm{~d} x^{2}+\right.$ $\left.\mathrm{d} y^{2}\right)$ for a local function $F$. In this coordinates, Equation (12) reads

$$
\partial_{x} \alpha_{y}-\partial_{y} \alpha_{x}=F\left(\alpha_{x}^{2}+\alpha_{y}^{2}\right)^{\frac{1}{2}},
$$

where we have written $\alpha^{\perp}=\alpha_{x} \mathrm{~d} x+\alpha_{y} \mathrm{~d} y$. Assuming $\alpha_{x}=0$ the previous equation admits the solution

$$
\alpha_{y}=e^{\int F \mathrm{~d} x+f(y)},
$$

where $f(y)$ is a local function depending only on $y$. Assuming on the other hand $\alpha_{y}=0$ the previous equation admits the solution

$$
\alpha_{x}=e^{-\int F \mathrm{~d} y+f(x)},
$$

where $f(x)$ is a local function depending only on $x$. Hence, we obtain the following result.

Proposition 4.11. Every solution to the constraint equations of General Relativity coupled to a cosmological constant is, at least locally, a solution to the constraint equations of an $\varepsilon \eta$-Einstein null contact structure with $\kappa=0$. 
We study now the $\varepsilon \eta$-Einstein condition on a case by case basis by distinguishing the signature of $g$ and the the causal character of the Reeb vector field.

4.1. Riemannian $\varepsilon \eta$-Einstein contact structures. We briefly review in this section the classification $\varepsilon \eta$-Einstein Riemannian contact structures, with the goal of constructing solutions of supergravity coupled to a tensor multiplet, as explained in Sections 5 and 6 . Riemannian contact structures on three-manifolds have been extensively studied in the literature, see $[11,33,38]$ and references therein. Adapting and refining the results of $[11,33,38]$ to our situation and conventions we obtain the following Theorem.

Theorem 4.12. [11, 33, 38] Let $(M, g, \alpha)$ be a three-dimensional complete and simply connected $\varepsilon \eta$-Einstein Riemannian contact metric manifold, $(M, g, \alpha) \in \operatorname{PCont}_{R}^{\varepsilon \eta}\left(\lambda^{2}, \kappa\right)$. Then one of the following holds:

- $(M, g, \alpha)$ is Sasakian. If $(M, g, \alpha)$ is in addition a Lie group equipped with a left-invariant $\varepsilon \eta$ Einstein Sasakian structure, then it is isomorphic ${ }^{1}$ to a left-invariant $\varepsilon \eta$-Einstein structure on:

(1) $\mathrm{SU}(2)$ if $\lambda^{2}=1+\kappa, \kappa>-1$.

(2) $\mathrm{H}_{3}$ if $\lambda^{2}=0, \kappa=-1$.

- $(M, g, \alpha)$ is non-Sasakian and isomorphic to a left-invariant $\varepsilon \eta$-Einstein structure on:

(1) $\mathrm{SU}(2)$ if $\lambda^{2}=-\kappa=\frac{1}{2}-\frac{1}{2} \mu^{2}$, with $0<\mu<1$ the positive eigenvalue of the tensor field $\mathfrak{h}$.

(2) $\widetilde{\mathrm{E}}(2)$ if $\lambda^{2}=\kappa=0$.

Proof. If $(M, g, \alpha)$ is Sasakian and not isomorphic to a Lie group equipped with a left-invariant structure $(g, \alpha)$ then we are done. On the other hand, reference [38] proves that a complete and simply connected $\varepsilon \eta$-Einstein non-Sasakian Riemannian contact three-manifold has a Lie group structure respect to which its $\varepsilon \eta$-Einstein structure is left-invariant. Therefore, we use the classification [44] of three-dimensional Riemannian Lie algebras to proceed on a case by case basis evaluating the $\varepsilon \eta$-Einstein condition. We distinguish between the Sasakian and the non-Sasakian cases.

- Sasakian case. First, if $(M, g, \alpha)$ is a Sasakian unimodular Lie group then there exists an orthonormal left-invariant frame $\left\{e_{1}, e_{2}, e_{3}\right\}$ whose associated Lie brackets satisfy [44]:

$$
\left[e_{2}, e_{3}\right]=\mu_{1} e_{1}, \quad\left[e_{3}, e_{1}\right]=\mu_{2} e_{2}, \quad\left[e_{1}, e_{2}\right]=\mu_{3} e_{3},
$$

for some real constants $\mu_{1}, \mu_{2}, \mu_{3} \in \mathbb{R}$. This immediately implies:

$$
\mathrm{d} e^{1}=-\mu_{1} e^{2} \wedge e^{3}, \quad \mathrm{~d} e^{2}=-\mu_{2} e^{3} \wedge e^{1}, \quad \mathrm{~d} e^{3}=-\mu_{3} e^{1} \wedge e^{2},
$$

where $\left\{e^{1}, e^{2}, e^{3}\right\}$ is the coframe dual to $\left\{e_{1}, e_{2}, e_{3}\right\}$. Expressing $\alpha=\alpha_{0} e^{0}+\alpha_{1} e^{1}+\alpha_{2} e^{2}$, the contact condition (we include the sign $s \in \mathbb{Z}_{2}$ in order to take into account orientation-reversing isometries) reads

what is equivalent to:

$$
* \alpha=s \mathrm{~d} \alpha,
$$

$$
\alpha_{1}=-s \mu_{1} \alpha_{1}, \quad \alpha_{2}=-s \mu_{2} \alpha_{2}, \quad \alpha_{3}=-s \mu_{3} \alpha_{3} .
$$

Furthermore, the non-zero components of the Ricci curvature tensor $\operatorname{Ric}^{g}$ read:

$$
\begin{array}{cl}
\operatorname{Ric}^{g}\left(e_{1}, e_{1}\right)=\frac{1}{2}\left(\mu_{1}^{2}-\mu_{2}^{2}-\mu_{3}^{2}+2 \mu_{2} \mu_{3}\right), & \operatorname{Ric}^{g}\left(e_{2}, e_{2}\right)=\frac{1}{2}\left(-\mu_{1}^{2}+\mu_{2}^{2}-\mu_{3}^{2}+2 \mu_{1} \mu_{3}\right), \\
\operatorname{Ric}^{g}\left(e_{3}, e_{3}\right)=\frac{1}{2}\left(-\mu_{1}^{2}-\mu_{2}^{2}+\mu_{3}^{2}+2 \mu_{1} \mu_{2}\right), & \operatorname{Ric}^{g}\left(e_{1}, e_{2}\right)=\operatorname{Ric}^{g}\left(e_{1}, e_{3}\right)=\operatorname{Ric}^{g}\left(e_{2}, e_{3}\right)=0 .
\end{array}
$$

We distinguish now the following subcases of the Sasakian unimodular case:

\footnotetext{
${ }^{1}$ Two $\varepsilon$-contact structures are isomorphic in the sense given at Definition 2.6.
} 
- Assume $\alpha_{1}, \alpha_{2}, \alpha_{3} \neq 0$. Then $\mu_{1}=\mu_{2}=\mu_{3}=-s$ and $\operatorname{Ric}^{g}=\frac{1}{2} g$, which follows from $\lambda^{2}=1$ and $\kappa=0$. Therefore, choosing $s=-1$ we conclude that $(M, g)$ is isometric to $\mathrm{SU}(2)$ equipped with a left-invariant metric.

- Assume $\alpha_{1}, \alpha_{2} \neq 0$ and $\alpha_{3}=0$. Again, $\mu_{1}, \mu_{2}=-s$. Since $\operatorname{Ric}\left(e_{1}, e_{2}\right)=0$, we obtain $\kappa=0$. Consequently, $\lambda^{2}=1$ which in turn implies, by equating $\operatorname{Ric}^{g}\left(e_{1}, e_{1}\right)=\operatorname{Ric}^{g}\left(e_{2}, e_{2}\right)=$ $\operatorname{Ric}^{g}\left(e_{3}, e_{3}\right)$, that $\mu_{3}=-s$. Taking $s=-1$, we recover the previous case and we conclude that $(M, g)$ is isometric to $\mathrm{SU}(2)$. A similar analysis holds when $\alpha_{1}, \alpha_{3} \neq 0$ and $\alpha_{2}=0$ and when $\alpha_{2}, \alpha_{3} \neq 0$ and $\alpha_{1}=0$.

- Assume $\alpha_{2}=\alpha_{3}=0$ and $\alpha_{1}^{2}=1$. Then, $\xi= \pm e_{1}$ and we obtain $\operatorname{Ric}^{g}\left(e_{1}, e_{1}\right)=\frac{1}{2}$ by the Sasaki condition. However, owing the fact that $\alpha_{1} \neq 0$ implies that $\mu_{1}=-s$, then we have that

$$
\operatorname{Ric}^{g}\left(e_{1}, e_{1}\right)=\frac{1}{2}-\frac{1}{2}\left(\mu_{2}-\mu_{3}\right)^{2}=\frac{1}{2} .
$$

Therefore $\mu_{2}=\mu_{3}=\mu \in \mathbb{R}$. Now, since $\operatorname{Ric}^{g}\left(e_{2}, e_{2}\right)=\operatorname{Ric}^{g}\left(e_{3}, e_{3}\right)=\frac{1}{2}\left(2 \lambda^{2}-1\right)$, we get the constraint

$$
\lambda^{2}=-s \mu .
$$

Taking $s=-1$, we conclude that for $\mu>0(M, g)$ is isometric to $\mathrm{SU}(2)$ and that for $\mu=0$ $(M, g)$ is isometric to $\mathrm{H}_{3}$. A completely similar analysis holds when $\alpha_{2}^{2}=1$ and $\alpha_{3}^{2}=1$.

Secondly, if $(M, g, \alpha)$ is a Sasakian non-unimodular Lie group then there exists an orthonormal left-invariant frame $\left\{e_{1}, e_{2}, e_{3}\right\}$ whose associated Lie brackets satisfy [44]:

$$
\left[e_{1}, e_{2}\right]=a e_{2}+b e_{3}, \quad\left[e_{1}, e_{3}\right]=c e_{2}+f e_{3}, \quad\left[e_{2}, e_{3}\right]=0,
$$

for real numbers $a, b, c, f \in \mathbb{R}$ satisfying that $a+f \neq 0$. These Lie brackets immediately imply:

$$
\mathrm{d} e^{1}=0, \quad \mathrm{~d} e^{2}=-a e^{1} \wedge e^{2}-c e^{1} \wedge e^{3}, \quad \mathrm{~d} e^{3}=-b e^{1} \wedge e^{2}-f e^{1} \wedge e^{3} .
$$

Therefore the contact condition imposes the following constraints:

$$
c \alpha_{2}+f \alpha_{3}=s \alpha_{2}, \quad-a \alpha_{2}-b \alpha_{3}=s \alpha_{3}, \quad \alpha_{1}=0 .
$$

The components of the Ricci curvature tensor in this orthonormal basis read:

$$
\begin{gathered}
\operatorname{Ric}^{g}\left(e_{1}, e_{1}\right)=-a^{2}-f^{2}-\frac{1}{2}(b+c)^{2}, \quad \operatorname{Ric}^{g}\left(e_{2}, e_{2}\right)=-a^{2}-\frac{b^{2}}{2}+\frac{c^{2}}{2}-f a, \\
\operatorname{Ric}^{g}\left(e_{3}, e_{3}\right)=-f^{2}+\frac{b^{2}}{2}-\frac{c^{2}}{2}-a f, \quad \operatorname{Ric}^{g}\left(e_{1}, e_{2}\right)=\operatorname{Ric}^{g}\left(e_{1}, e_{3}\right)=0, \quad \operatorname{Ric}^{g}\left(e_{2}, e_{3}\right)=-a c-b f .
\end{gathered}
$$

In particular, we have:

$$
\operatorname{Ric}^{g}\left(e_{2}, e_{2}\right)+\operatorname{Ric}^{g}\left(e_{3}, e_{3}\right)=-a^{2}-2 a f-f^{2}=-(a+f)^{2} .
$$

Imposing the $\varepsilon \eta$-Einstein condition, and taking into account that $\alpha_{2}^{2}+\alpha_{3}^{2}=1$ by the contact condition, we obtain $\operatorname{Ric}^{g}\left(e_{2}, e_{2}\right)+\operatorname{Ric}^{g}\left(e_{3}, e_{3}\right)=\lambda^{2}+\kappa-\kappa \alpha_{2}^{2}-\kappa \alpha_{3}^{2}=\lambda^{2}$, which in turn yields $\lambda^{2}=-(a+f)^{2}<0$. Hence, non-unimodular Riemannian groups admit no left-invariant $\varepsilon \eta$-Einstein structures.

- If $(M, g, \alpha)$ is non-Sasakian, then [38] shows that the $\varepsilon \eta$-Einstein condition implies $\lambda^{2}=-\kappa$. Furthermore, $[11,38]$ prove that there exists a frame $\{\xi, X, \phi(X)\}$, where $X$ is an eigenvector of $\mathfrak{h}$ with positive eigenvalue, whose Lie brackets satisfy:

$$
[\xi, X]=\frac{1}{2}(1+\mu) \phi(X), \quad[\xi, \phi(X)]=-\frac{1}{2}(1-\mu) X, \quad[X, \phi(X)]=\xi,
$$

where $\mu$ is the positive eigenvalue of $\mathfrak{h}$. Comparing the previous Lie brackets to Milnor's classification, we obtain that $M$ must be isometric to $\mathrm{SU}(2)$ when $\mu<1$ and isometric to $\widetilde{\mathrm{E}}(2)$ when $\mu=1$. Finally, using Proposition 2.19, we find the relation $\mu^{2}=1-2 \lambda^{2}$. 
4.2. Lorentzian $\varepsilon \eta$-Einstein structures with time-like Reeb vector field. Let $(M, g, \alpha,-1) \in$ $\operatorname{PCont}_{L}^{\varepsilon \eta}\left(-1, \lambda^{2}, \kappa\right)$ be an $\varepsilon$-contact metric manifold $M$ with time-like vector field. Note that, by Definition $4.1,(g, \alpha, \varepsilon)$ is $\varepsilon \eta$-Einstein if and only if it satisfies

$$
\operatorname{Ric}^{g}=\frac{1}{2}\left(-\lambda^{2}+\kappa\right) \chi+\kappa \alpha \otimes \alpha,
$$

where $\lambda \in \mathbb{R}$ and $\kappa \geq 0$.

Remark 4.13. The Lorentzian $\varepsilon \eta$-Einstein condition with time-like Reeb vector field has a natural physical interpretation in the context of General Relativity. Indeed, it is an Einstein-like equation, that is, it can be written as follows:

$$
\mathrm{G}(g)=\mathrm{T}(g, \alpha)
$$

where $\mathrm{G}(g)=\mathrm{Ric}^{g}-\frac{1}{2} \mathrm{R}^{g} g$ denotes the Einstein tensor and $\mathrm{T}(g, \alpha) \in \Gamma\left(T^{*} N \odot T^{*} N\right)$ is the energy-momentum tensor of Einstein's equations, given in this case by

$$
\mathrm{T}(g, \alpha)=\frac{1}{4}\left(\lambda^{2}+\kappa\right) \chi+\kappa \alpha \otimes \alpha .
$$

Interestingly enough, $\mathrm{T}(g, \alpha)$ corresponds with the energy-momentum tensor of a perfect fluid whose speed is prescribed by $\xi$ and whose pressure $p$ and rest-frame mass density $o$ are constant and given by:

$$
p=\frac{\lambda^{2}+\kappa}{4}, \quad o=\frac{3 \kappa-\lambda^{2}}{4} .
$$

Hence, the $\varepsilon \eta$-Einstein condition in Lorentzian signature with $\varepsilon=-1$ corresponds with the Einstein's General Relativity equations for a Lorentzian metric coupled to a perfect fluid with velocity prescribed by the Reeb vector field. This interpretation allows to apply the extensive literature dedicated to the study of perfect-fluid space-times, see for instance [31] and references therein, to the study of Lorentzian $\varepsilon \eta$-Einstein $\varepsilon$-contact structures.

We prove now that a Lorentzian $\varepsilon \eta$-Einstein contact structure $(g, \alpha,-1)$ on a connected and simply connected complete ${ }^{2}$ three-dimensional Lorentzian manifold $(M, g)$ is either Sasakian or isometric to a Lie group equipped with a Lorentzian left-invariant contact structure $(g, \alpha,-1)$. We proceed analogously to the Riemannian case [11]. We prove first existence of a special type of $\varepsilon$-contact frame, particularly convenient for computations.

Lemma 4.14. Let $(M, g, \alpha,-1) \in \operatorname{PCont}_{L}^{\varepsilon \eta}\left(-1, \lambda^{2}, \kappa\right)$ be a simply connected Lorentzian $\varepsilon \eta$-Einstein $\varepsilon$-contact metric three-manifold. Then, there exists a global orthonormal frame $\{\xi, X, \phi(X)\}$ such that $\mathfrak{h}(X)=\mu X$ and $\mathfrak{h}(\phi(X))=-\mu \phi(X)$, where:

$$
\mu=\sqrt{1-\left(\lambda^{2}+\kappa\right)} .
$$

In particular, $\lambda^{2}+\kappa \leq 1$.

Proof. First notice that since $M$ is simply connected and three-dimensional it is parallelizable, whence it admits nowhere vanishing vector fields. If $(g, \alpha)$ is Sasakian then $\mathfrak{h}=0$ and $\lambda^{2}+\kappa=1$, see Remark 4.5, and the statement is trivial. We consider thus the non-Sasakian case. For every

\footnotetext{
${ }^{2}$ We say that an $\varepsilon$-contact metric three-manifold $(N, \chi, \alpha, \varepsilon=-1)$ is complete if all elements of any $\varepsilon$-contact frame are complete on $N$.
} 
nowhere vanishing space-like vector field $Y \in \mathfrak{X}(M)$ of unit norm, $\{\xi, Y, \phi(Y)\}$ is a global $\varepsilon$-contact frame. Furthermore:

$$
g\left(\mathfrak{h}^{2}(Y), \xi\right)=0, \quad g\left(\mathfrak{h}^{2}(Y), \phi(Y)\right)=0
$$

since $g\left(\mathfrak{h}^{2}(Y), \phi(Y)\right)=g\left(Y, \mathfrak{h}^{2}(\phi(Y))\right)=g\left(Y, \phi\left(\mathfrak{h}^{2}(Y)\right)\right)=-g\left(\mathfrak{h}^{2}(Y), \phi(Y)\right)$. Therefore:

$$
\mathfrak{h}^{2}(Y)=\sigma^{2} Y=-\sigma^{2} \phi^{2}(Y)
$$

for some $\sigma \in C^{\infty}(M)$. Therefore, $\operatorname{Tr}\left(\mathfrak{h}^{2}\right)=2 \sigma^{2}$. Since $(M, g, \alpha,-1) \in \mathrm{PCont}_{L}^{\varepsilon \eta}\left(-1, \lambda^{2}, \kappa\right)$, combining Proposition 2.19 and Remark 4.5 we obtain that $\sigma=\sqrt{1-\left(\lambda^{2}+\kappa\right)}$. Since $(M, g, \alpha,-1)$ is not Sasakian we have $\lambda^{2}+\kappa<1$ whence $\mu$ is strictly positive and corresponds with the positive eigenvalue of $\mathfrak{h}$.

Working in the special global $\varepsilon$-contact frame introduced in Lemma 4.14 we prove that every nonSasakian $\varepsilon \eta$-Einstein connected and simply-connected Lorentzian three-dimensional manifold is a Lie group equipped with a left-invariant $\varepsilon \eta$-Einstein structure.

Proposition 4.15. Let $(M, g, \alpha,-1) \in \mathrm{PCont}_{L}^{\varepsilon \eta}\left(-1, \lambda^{2}, \kappa\right)$ be simply connected with $\lambda^{2}+\kappa<1$ (that is, $(M, g, \alpha)$ is not Sasakian). Then, the following Lie brackets hold in the special $\varepsilon$-contact frame $\{\xi, X, \phi(X)\}$ described in Lemma 4.14 :

$$
[\xi, X]=\frac{1}{2}\left(1+\sqrt{1-2 \lambda^{2}}\right) \phi(X), \quad[\xi, \phi(X)]=\frac{1}{2}\left(-1+\sqrt{1-2 \lambda^{2}}\right) X, \quad[X, \phi(X)]=-\xi .
$$

Furthermore, $\lambda^{2}=\kappa$ and $0 \leq \lambda^{2}=\kappa<\frac{1}{2}$ since $\kappa \geq 0$.

Proof. Propositions 2.13 and 2.14 imply that:

$$
\nabla_{\xi} \xi=0, \quad \nabla_{X} \xi=-\frac{1}{2}(1+\mu) \phi(X), \quad \nabla_{\phi(X)} \xi=\frac{1}{2}(1-\mu) X
$$

On the other hand, we have that $g\left(\nabla_{X} X, \xi\right)=-g\left(X, \nabla_{X} \xi\right)=0$ and

$$
g\left(\nabla_{\phi(X)} \phi(X), \xi\right)=-g\left(\phi(X), \nabla_{\phi(X)} \xi\right)=0 .
$$

Therefore, $\nabla_{X} X=c \phi(X)$ and $\nabla_{\phi(X)} \phi(X)=e X$ for some functions $c, e \in C^{\infty}(M)$. Similarly, $g\left(\nabla_{\xi} X, \xi\right)=g\left(\nabla_{\xi}(\phi(X)), \xi\right)=0$, whence $\nabla_{\xi} X=\beta \phi(X)$ and $\nabla_{\xi} \phi(X)=-\beta X$ for a function $\beta \in C^{\infty}(M)$. Furthermore, we have:

$$
\begin{gathered}
g\left(\nabla_{X} \phi(X), \xi\right)=-g\left(\phi(X), \nabla_{X} \xi\right)=\frac{1}{2}(1+\mu), \quad g\left(\nabla_{\phi(X)} X, \xi\right)=-g\left(X, \nabla_{\phi(X)} \xi\right)=\frac{1}{2}(\mu-1), \\
g\left(\nabla_{X} \phi(X), X\right)=-g\left(\phi(X), \nabla_{X} X\right)=-c, \quad g\left(\nabla_{\phi(X)} X, \phi(X)\right)=-g\left(X, \nabla_{\phi(X)} \phi(X)\right)=-e .
\end{gathered}
$$

Summarizing:

$$
\begin{gathered}
\nabla_{\xi} \xi=0, \quad \nabla_{X} \xi=-\frac{1}{2}(1+\mu) \phi(X), \quad \nabla_{\phi(X)} \xi=\frac{1}{2}(1-\mu) X \\
\nabla_{\xi} X=\beta \phi(X), \quad \nabla_{X} X=c \phi(X), \quad \nabla_{\phi(X)} X=\frac{1}{2}(1-\mu) \xi-e \phi(X), \\
\nabla_{\xi} \phi(X)=-\beta X, \quad \nabla_{X} \phi(X)=-\frac{1}{2}(1+\mu) \xi-c X, \nabla_{\phi(X)} \phi(X)=e X,
\end{gathered}
$$

which implies:

$$
[\xi, X]=\left(\beta+\frac{1}{2}+\frac{\mu}{2}\right) \phi(X), \quad[\xi, \phi(X)]=\left(-\beta-\frac{1}{2}+\frac{\mu}{2}\right) X, \quad[X, \phi(X)]=-\xi-c X+e \phi(X),
$$

by using the torsion-free property of $\nabla$. Making use of the previous equations, we compute:

$$
\mathrm{R}^{g}(\xi, X) \xi=\left(\mu \beta-\frac{1}{4}+\frac{\mu^{2}}{4}\right) X, \quad \mathrm{R}^{g}(X, \phi(X)) \xi=e \mu X-c \mu \phi(X) .
$$


By comparing with Lemma 4.6, we conclude that $\mu \beta=\mu c=\mu e=0$. Since $\mu \neq 0$ by assumption, then $\beta=c=e=0$. Finally, by $\operatorname{Ric}^{g}(X, X)=\operatorname{Ric}^{g}(\phi(X), \phi(X))=0$ we get $\lambda^{2}=\kappa$. Hence, $\mu=\sqrt{1-2 \lambda^{2}}$ and we conclude.

Remark 4.16. From the proof of Proposition 4.15 we extract the following covariant derivatives of the $\varepsilon$-contact frame of special type described in Lemma 4.14:

$$
\begin{gathered}
\nabla_{\xi} \xi=0, \quad \nabla_{X} \xi=-\frac{1}{2}\left(1+\sqrt{1-2 \lambda^{2}}\right) \phi(X), \quad \nabla_{\phi(X)} \xi=\frac{1}{2}\left(1-\sqrt{1-2 \lambda^{2}}\right) X \\
\nabla_{\xi} X=0, \quad \nabla_{X} X=0, \quad \nabla_{\phi(X)} X=\frac{1}{2}\left(1-\sqrt{1-2 \lambda^{2}}\right) \xi \\
\nabla_{\xi} \phi(X)=0, \quad \nabla_{X} \phi(X)=-\frac{1}{2}\left(1+\sqrt{1-2 \lambda^{2}}\right) \xi, \quad \nabla_{\phi(X)} \phi(X)=0 .
\end{gathered}
$$

Proposition 4.17. Let $(M, g, \alpha,-1) \in \operatorname{PCont}_{L}^{\varepsilon \eta}\left(-1, \lambda^{2}, \kappa\right)$ be a complete and simply connected three-dimensional Lorentz $\varepsilon \eta$-Einstein contact manifold. Then, one of the following holds:

- $(M, g, \alpha,-1)$ is Sasakian.

- $(M, g, \alpha,-1)$ is non-Sasakian and isomorphic to one of the following Lie groups equipped with a left-invariant $\varepsilon \eta$-Einstein structure $(g, \alpha,-1)$ :

(1) $\widetilde{\mathrm{Sl}}(2, \mathbb{R})$ when $\frac{1}{2}>\lambda^{2}=\kappa>0$.

(2) $\widetilde{\mathrm{E}}(1,1)$ when $\lambda^{2}=\kappa=0$.

Proof. Assume $(M, g, \alpha,-1)$ is non-Sasakian. By Proposition 4.15, there exists a global orthonormal frame $\{\xi, X, \phi(X)\}$ such that:

$$
[\xi, X]=\frac{1}{2}\left(1+\sqrt{1-2 \lambda^{2}}\right) \phi(X), \quad[\xi, \phi(X)]=\frac{1}{2}\left(-1+\sqrt{1-2 \lambda^{2}}\right) X, \quad[X, \phi(X)]=-\xi,
$$

such that $\lambda^{2}=\kappa$ and $0 \leq \lambda^{2}=\kappa<\frac{1}{2}$. Using [51, Proposition 1.9], the fact that $M$ admits a global frame with constant structure functions implies that $(M, g, \alpha)$ has a Lie group structure (canonical after fixing an identity point) respect to which $\{\xi, X, \phi(X)\}$ is left-invariant. Using the classification of connected and simply connected three-dimensional Lie groups summarized in Appendix A we conclude that $(M, g)$ is of type $\mathfrak{g}_{3}$ when $\frac{1}{2}>\lambda^{2}=\kappa>0$ by identifying:

$$
\xi=e_{0}, \quad e_{1}=X, \quad e_{2}=\phi(X) .
$$

In particular, we have:

$$
a=\frac{1}{2}\left(1-\sqrt{1-2 \lambda^{2}}\right), \quad b=\frac{1}{2}\left(1+\sqrt{1-2 \lambda^{2}}\right), \quad c=1 .
$$

where $a, b$ and $c$ are the real parameters appearing in case $\mathfrak{g}_{3}$ of Theorem A.1. Hence $(M, g)$ is isometric to $\widetilde{\mathrm{Sl}}(2, \mathbb{R})$ equipped with a left-invariant metric. On the other hand, when $\lambda^{2}=\kappa=0$, identifying:

$$
e_{0}=-\xi, \quad e_{1}=-\phi(X), \quad e_{2}=-X,
$$

we obtain that $(M, g)$ is isometric to $\widetilde{\mathrm{E}}(1,1)$ endowed with a left-invariant metric.

For Sasakian structures, we obtain the following result.

Lemma 4.18. Let $(\mathrm{G}, g, \alpha,-1) \in \operatorname{PCont}_{L}^{\varepsilon \eta}\left(-1, \lambda^{2}, 1-\lambda^{2}\right)$ be a left-invariant Sasakian Lorentzian $\varepsilon \eta$-Einstein contact structure on a simply connected Lie group $\mathrm{G}$. Then, according to the classification of connected and simply connected 3-dimensional Lie groups given in Theorem A.1, one of the following holds:

- $\mathrm{G}$ is of type $\mathfrak{g}_{3}$. In particular, we have that $(\mathrm{G}, g, \alpha,-1)$ is isomorphic to a left-invariant $\varepsilon \eta$ Einstein structure on: 
(1) $\widetilde{\mathrm{Sl}}(2, \mathbb{R})$ if $1 \geq \lambda^{2}>0$.

(2) $\mathrm{H}_{3}$ if $\lambda^{2}=0$.

- $\mathrm{G}$ is of type $\mathfrak{g}_{6}$ and $1 \geq \lambda^{2}>0$.

Proof. Let $\{\xi, X, \phi(X)\}$ be a left-invariant $\varepsilon$-contact frame on $(\mathrm{G}, \xi, \alpha)$. The proof of Proposition 4.15 shows that the following holds:

$$
\begin{gathered}
\nabla_{\xi} \xi=0, \quad \nabla_{X} \xi=-\frac{1}{2} \phi(X), \quad \nabla_{\phi(X)} \xi=\frac{1}{2} X \\
\nabla_{\xi} X=\beta \phi(X), \quad \nabla_{X} X=c \phi(X), \quad \nabla_{\phi(X)} X=\frac{1}{2} \xi-e \phi(X), \\
\nabla_{\xi} \phi(X)=-\beta X, \quad \nabla_{X} \phi(X)=-\frac{1}{2} \xi-c X, \quad \nabla_{\phi(X)} \phi(X)=e X, \\
{[\xi, X]=\left(\beta+\frac{1}{2}\right) \phi(X), \quad[\xi, \phi(X)]=-\left(\beta+\frac{1}{2}\right) X, \quad[X, \phi(X)]=-\xi-c X+e \phi(X),}
\end{gathered}
$$

where $\beta, c, e \in \mathbb{R}$. Imposing the Jacobi identity, we obtain the constraint

$$
e\left(\beta+\frac{1}{2}\right) X+c\left(\beta+\frac{1}{2}\right) \phi(X)=0 .
$$

Hence, either $c=e=0$ or $\beta=-\frac{1}{2}$. We consider these cases separately.

- Assume $c=e=0$. Imposing that $(\mathrm{G}, g, \alpha) \in \operatorname{PCont}_{L}^{\varepsilon \eta}\left(-1, \lambda^{2}, 1-\lambda^{2}\right)$, we obtain the condition $\operatorname{Ric}^{g}(X, X)=\operatorname{Ric}^{g}(\phi(X), \phi(X))=\frac{1}{2}-\lambda^{2}$, which is equivalent to

$$
\beta=\lambda^{2}-\frac{1}{2}
$$

Hence, the Lie brackets of $\{\xi, X, \phi(X)\}$ reduce to:

$$
[\xi, X]=\lambda^{2} \phi(X), \quad[\xi, \phi(X)]=-\lambda^{2} X, \quad[X, \phi(X)]=-\xi .
$$

For $\lambda^{2}>0$, if $\left\{e_{0}, e_{1}, e_{2}\right\}$ denotes the orthonormal basis used at Theorem A.1, identifying $e_{0}=\xi$, $e_{1}=X$ and $e_{2}=\phi(X)$, we conclude that $(\mathrm{G}, g)$ is isometric to $\widetilde{\mathrm{Sl}}(2, \mathbb{R})$ endowed with a leftinvariant metric. Similarly, for $\lambda^{2}=0$, setting $e_{0}=-\xi, e_{1}=X, e_{2}=\phi(X)$ we conclude that $(\mathrm{G}, g)$ is isometric to $\mathrm{H}_{3}$ equipped with a left-invariant metric.

- If $\beta=-\frac{1}{2}$, the only non-trivial constraint from the $\varepsilon \eta$-Einstein condition is given by $\operatorname{Ric}^{g}(X, X)=$ $\operatorname{Ric}^{g}(\phi(X), \phi(X))=\frac{1}{2}-\lambda^{2}$, which is equivalent to

$$
c^{2}+e^{2}=\lambda^{2} .
$$

Consequently, we obtain:

$$
[\xi, X]=0, \quad[\xi, \phi(X)]=0, \quad[X, \phi(X)]=-\xi-c \phi(X)+e X,
$$

with $c^{2}+e^{2}=\lambda^{2}$. We assume $\lambda^{2} \neq 0$, since otherwise we return to the previous bullet point. Parametrizing $e=-|\lambda| \cos \theta$ and $c=|\lambda| \sin \theta$ for some angle $\theta \in \mathbb{R}$, we consider the following orthogonal change of basis:

$$
[\xi, \bar{X}, \phi(\bar{X})]^{T}=\left[\begin{array}{ccc}
1 & 0 & 0 \\
0 & \cos \theta & \sin \theta \\
0 & -\sin \theta & \cos \theta
\end{array}\right][\xi, X, \phi(X)]^{T},
$$

finding:

$$
[\xi, \bar{X}]=0, \quad[\xi, \phi(\bar{X})]=0, \quad[\bar{X}, \phi(\bar{X})]=-\xi-|\lambda| \bar{X} .
$$

Defining $e_{0}=\xi, e_{1}=\phi(\bar{X})$ and $e_{2}=\bar{X}$ we conclude, via Theorem A.1, that the previous Lie brackets correspond to those of a Lie algebra type $\mathfrak{g}_{6}$. 
We may summarize the information provided in Proposition 4.17 and Lemma 4.18 in the following Theorem.

Theorem 4.19. A three-dimensional connected and simply connected Lie group $G$ admits a leftinvariant $\varepsilon \eta$-Einstein contact structure $(g, \alpha)$ with time-like Reeb vector field if and only if $(\mathrm{G}, g, \alpha)$ is isomorphic, through a possibly orientation-reversing isometry, to one of the items listed in the following table in terms of the orthonormal frame $\left\{e_{0}, e_{1}, e_{2}\right\}$ appearing in Theorem A.1:

\begin{tabular}{|c|c|c|c|c|c|}
\hline $\mathfrak{g}$ & Structure constants & $\alpha$ & $\eta$-Einstein constants & $G$ & Sasakian \\
\hline \multirow{4}{*}{$\mathfrak{g}_{3}$} & $\frac{1}{2}>a=1-b>0, c=1$ & $\alpha=e^{0}$ & $\lambda^{2}=\kappa=2 b(1-b)$ & $\widetilde{\mathrm{Sl}}(2, \mathbb{R})$ & No \\
\cline { 2 - 6 } & $a=c=1, b=0$ & $\alpha=-e^{0}$ & $\lambda^{2}=\kappa=0$ & $\widetilde{\mathrm{E}}(1,1)$ & No \\
\cline { 2 - 6 } & $1 \geq a=b>0, c=1$ & $\alpha=e^{0}$ & $\lambda^{2}=1-\kappa=a$ & $\widetilde{\mathrm{Sl}}(2, \mathbb{R})$ & Yes \\
\cline { 2 - 6 } & $a=b=0, c=-1$ & $\alpha=-e^{0}$ & $\lambda^{2}=0, \kappa=1$ & $\mathrm{H}_{3}$ & Yes \\
\hline $\mathfrak{g}_{6}$ & $b=1, c=d=0,1 \geq a^{2}>0$ & $\alpha=e^{0}$ & $\lambda^{2}=1-\kappa=a^{2}$, & $\mathfrak{G}_{6}$ & Yes \\
\hline
\end{tabular}

Furthermore, if $(M, g, \alpha,-1) \in \mathrm{PCont}_{L}^{\varepsilon \eta}\left(-1, \lambda^{2}, \kappa\right)$ is complete and not Sasakian then it is a Lie group equipped with a left-invariant Lorentzian contact structure and isomorphic to a left-invariant $\varepsilon \eta$-Einstein structure on either $\widetilde{\mathrm{Sl}}(2, \mathbb{R})$ when $\frac{1}{2}>\lambda^{2}=\kappa>0$ or on $\widetilde{\mathrm{E}}(1,1)$ when $\lambda^{2}=\kappa=0$.

Proof. Follows from Proposition 4.17 and Lemma 4.18 upon use of Theorem A.1.

4.3. Lorentzian case with space-like Reeb vector field. In this subsection we classify all leftinvariant $\varepsilon \eta$-Einstein para-contact structures on three-dimensional simply-connected Lie groups. Let $(N, \chi, \alpha, \varepsilon=1) \in \operatorname{PCont}_{L}^{\varepsilon \eta}(\varepsilon=1, \lambda, \kappa)$ be an oriented and time-oriented Lorentzian $\varepsilon$-contact metric manifold. By Definition 4.1, $(\chi, \alpha, \varepsilon=1)$ is $\varepsilon \eta$-Einstein if and only if it satisfies:

$$
\operatorname{Ric}^{\chi}=-\frac{1}{2}\left(\lambda^{2}+\kappa\right) \chi+\kappa \alpha \otimes \alpha
$$

for real constants $\lambda \in \mathbb{R}$ and $\kappa \geq 0$.

Remark 4.20. Note that, in contrast to the case $\mathfrak{s}_{g} \varepsilon=1$, the endomorphism $\mathfrak{h}$ associated to a para-contact metric structure may not be diagonalizable, whence the techniques usted to classify $\varepsilon \eta$-Einstein $\varepsilon$-contact metric three manifolds with $\mathfrak{s}_{g} \varepsilon=1$ are a priori not applicable here.

Theorem 4.21. A three-dimensional connected and simply connected Lie group $G$ admits a leftinvariant $\varepsilon \eta$-Einstein para-contact structure $(g, \alpha)$ if and only if $(\mathrm{G}, g, \alpha)$ is isomorphic, through a possibly orientation-reversing isometry, to one of the items listed in the following table in terms of the orthonormal frame $\left\{e_{0}, e_{1}, e_{2}\right\}$ appearing in Theorem A.1: 


\begin{tabular}{|c|c|c|c|c|c|}
\hline $\mathfrak{g}$ & Structure constants $\left(s \in \mathbb{Z}_{2}\right)$ & $\alpha$ & $\eta$-Einstein constants & $\mathrm{G}$ & Sasakian \\
\hline \multirow{5}{*}{$\mathfrak{g}_{3}$} & $a=s, b=c, s c \geq 1$ & $\alpha= \pm e^{1}$ & $\lambda^{2}=s c, \kappa=s c-1$ & $\widetilde{\mathrm{Sl}}(2, \mathbb{R})$ & Yes \\
\hline & $b=s, a=c, s c \geq 1$ & $\alpha= \pm e^{2}$ & $\lambda^{2}=s c, \kappa=s c-1$ & $\widetilde{\mathrm{Sl}}(2, \mathbb{R})$ & Yes \\
\hline & $b=0, a=c=s$ & $-\alpha_{0}^{2}+\alpha_{1}^{2}=1$ & $\lambda^{2}=0, \kappa=0$ & $\widetilde{\mathrm{E}}(1,1)$ & No \\
\hline & $c=0, a=b=s$ & $\alpha_{1}^{2}+\alpha_{2}^{2}=1$ & $\lambda^{2}=0, \kappa=0$ & $\widetilde{\mathrm{E}}(2)$ & No \\
\hline & $a=b=c=s$ & $-\alpha_{0}^{2}+\alpha_{1}^{2}+\alpha_{2}^{2}=1$ & $\lambda^{2}=1, \kappa=0$ & $\widetilde{\mathrm{Sl}}(2, \mathbb{R})$ & Yes \\
\hline \multirow[b]{2}{*}{$\mathfrak{g}_{6}$} & $a=b=0, d^{2} \geq 1, c=-s$ & $\alpha_{2}^{2}=1$ & $\lambda^{2}=d^{2}, \kappa=d^{2}-1$ & \multirow[b]{2}{*}{$\mathfrak{G}_{6}$} & Yes \\
\hline & $\begin{array}{c}b=-\mu a \neq 0, d=-a+\mu s \\
c=-s+\mu a, \mu \in \mathbb{Z}_{2}\end{array}$ & $\begin{array}{c}a \alpha_{2}=(-s+\mu a) \alpha_{0} \\
\alpha_{2}^{2}=1+\alpha_{0}^{2}\end{array}$ & $\lambda^{2}=1, \kappa=0$ & & Yes \\
\hline
\end{tabular}

Proof. We proceed on a case by case basis by checking which of the items appearing in Theorem A.1 admits an $\varepsilon \eta$-Einstein para-contact metric structure $(g, \alpha)$. For this, we will exploit the formulae presented in Appendix B.

- Case $\mathfrak{g}_{1}$. In the orthonormal frame given in Appendix A, Equation $* \alpha=-s \mathrm{~d} \alpha$ is equivalent to

$$
\begin{gathered}
\left(-a \alpha_{1}-b \alpha_{2}\right) e^{0} \wedge e^{1}+\left(a \alpha_{0}+b \alpha_{1}+a \alpha_{2}\right) e^{0} \wedge e^{2}+\left(b \alpha_{0}-a \alpha_{1}\right) e^{1} \wedge e^{2} \\
=s\left(\alpha_{0} e^{1} \wedge e^{2}+\alpha_{1} e^{0} \wedge e^{2}-\alpha_{2} e^{0} \wedge e^{1}\right)
\end{gathered}
$$

Non-trivial solutions for $\alpha$ exist only if $b=s$, which implies $\alpha_{1}=0$, since $a \neq 0$. This implies in turn $\alpha_{0}+\alpha_{2}=0$, which is incompatible with the constraint $-\alpha_{0}^{2}+\alpha_{2}^{2}=1$. Hence $\mathfrak{g}_{1}$ does not admit para-contact metric structures.

- Case $\mathfrak{g}_{2}$. In the orthonormal frame given in Appendix A, Equation $* \alpha=-s \mathrm{~d} \alpha$ is equivalent to

$$
\begin{aligned}
a \alpha_{1} e^{0} & \wedge e^{2}+\left(c \alpha_{0}-b \alpha_{2}\right) e^{0} \wedge e^{1}+\left(c \alpha_{2}+b \alpha_{0}\right) e^{1} \wedge e^{2} \\
& =s\left(\alpha_{0} e^{1} \wedge e^{2}+\alpha_{1} e^{0} \wedge e^{2}-\alpha_{2} e^{0} \wedge e^{1}\right) .
\end{aligned}
$$

This system of equations implies $c^{2}+(b-s)^{2}=0$, which is equivalent to $c=0$ and $b=s$. Since the value $c=0$ is forbidden for $\mathfrak{g}_{2}$ Lie algebras, we conclude that there are no para-contact metric structures on this type of Lie algebras.

- Case $\mathfrak{g}_{3}$. In the orthonormal frame given in Appendix A, Equation $* \alpha=-s \mathrm{~d} \alpha$ is equivalent to

$$
c \alpha_{0} e^{1} \wedge e^{2}+a \alpha_{1} e^{0} \wedge e^{2}-b \alpha_{2} e^{0} \wedge e^{1}=s\left(\alpha_{0} e^{1} \wedge e^{2}+\alpha_{1} e^{0} \wedge e^{2}-\alpha_{2} e^{0} \wedge e^{1}\right)
$$

If $\alpha_{0}, \alpha_{1}, \alpha_{2} \neq 0$, then we have $a=b=c=s$ and $-\alpha_{0}^{2}+\alpha_{1}^{2}+\alpha_{2}^{2}=1$. If $\alpha_{0}=0$ and $\alpha_{1}, \alpha_{2} \neq 0$, then $\alpha_{1}^{2}+\alpha_{2}^{2}=1, a=b=s$ and $c$ is unconstrained. If $\alpha_{1}=0, \alpha_{0}, \alpha_{2} \neq 0$ (resp. $\alpha_{2}=0, \alpha_{0}, \alpha_{1} \neq 0$ ) we have $-\alpha_{0}^{2}+\alpha_{2}^{2}=1, b=c=s$ and $a$ unconstrained (resp. $-\alpha_{0}^{2}+\alpha_{1}^{2}=1$, $a=c=s$ and $b$ unconstrained). If $\alpha_{0}=\alpha_{1}=0$ (resp. $\alpha_{0}=\alpha_{2}=0$ ), then $\alpha_{2}^{2}=1, b=s$ and $a, c$ are unconstrained (resp. $\alpha_{1}^{2}=1, a=s$ and $b, c$ unconstrained). Finally, we remark that $a=b=0$ is never allowed, since it implies $\alpha_{1}=\alpha_{2}=0$, whence $\alpha_{0}^{2}=-1$. 
We compute now the Ricci curvature. We obtain:

$$
\begin{array}{ll}
\operatorname{Ric}^{g}\left(e_{0}, e_{0}\right)=\frac{c^{2}}{2}+b a-\frac{b^{2}}{2}-\frac{a^{2}}{2}, & \operatorname{Ric}^{g}\left(e_{0}, e_{1}\right)=0, \\
\operatorname{Ric}^{g}\left(e_{1}, e_{1}\right)=\frac{b^{2}}{2}+\frac{c^{2}}{2}-\frac{a^{2}}{2}-c b, & \operatorname{Ric}^{g}\left(e_{0}, e_{2}\right)=0, \\
\operatorname{Ric}^{g}\left(e_{2}, e_{2}\right)=\frac{a^{2}}{2}-a c+\frac{c^{2}}{2}-\frac{b^{2}}{2}, & \operatorname{Ric}^{g}\left(e_{1}, e_{2}\right)=0 .
\end{array}
$$

We proceed now on a case by case basis:

(1) If $\alpha_{0}, \alpha_{1}, \alpha_{2} \neq 0$, and hence $a=b=c=s$, the $\varepsilon \eta$-Einstein condition reduces to

$$
\lambda^{2}=1, \quad \kappa=0 .
$$

which follows by direct computation from (14).

(2) If $\alpha_{0}=0$ and $\alpha_{1}, \alpha_{2} \neq 0$, we have $a=b=s$ and $c$ unconstrained. Then $\operatorname{Ric}^{g}\left(e_{1}, e_{2}\right)=0$ implies $\kappa=0$, which solves all off-diagonal $\varepsilon \eta$-Einstein equations. The diagonal components of the $\varepsilon \eta$-Einstein equations are equivalent to

$$
\lambda^{2}=c^{2} \quad c^{2}=s c .
$$

Hence, either $c \neq 0$, which implies $c=s$ and we are back to point (1), or $c=0$, in which case $\lambda=0$. The cases $\alpha_{1}=0\left(\alpha_{2}=0\right)$ and $\alpha_{0}, \alpha_{2} \neq 0\left(\alpha_{0}, \alpha_{1} \neq 0\right)$ follow analogously.

(3) We consider now $\alpha_{0}=\alpha_{1}=0$ and $\alpha_{2}^{2}=1$, so that $b=s$ and $a, c$ unconstrained. In this case, using the formulae of Appendix B the $\varepsilon \eta$-Einstein condition can be found to imply:

$$
(c-a)(s-(c+a))=0,
$$

whence either $c=a$ or $a+c=s$. If $a=c$ then the $\varepsilon \eta$-Einstein equations are equivalent to $a=s(1+\kappa)=c$ and $\lambda^{2}=1+\kappa$. Therefore, $\lambda^{2}=s c$. On the other hand, if $a+c=s$ then the $\varepsilon \eta$-Einstein condition implies $\kappa=-\lambda^{2}$. However, $\kappa \geq 0$ by the definition of $\varepsilon \eta$-Einstein structure, whence $\lambda=\kappa=0$ and the $\varepsilon \eta$-Einstein condition reduces to $a(a-s)=0$. Since $a=0$ is not allowed if $b=s$ by the type of algebra $\mathfrak{g}_{3}$, we conclude that $a=s$. The case $\alpha_{0}=\alpha_{2}=0$ and $\alpha_{1}^{2}=1$ follows now along similar lines.

- Case $\mathfrak{g}_{4}$. In the orthonormal frame given in Appendix A, Equation $* \alpha=-s \mathrm{~d} \alpha$ is equivalent to

$$
\begin{gathered}
a \alpha_{1} e^{0} \wedge e^{2}+\left(-(2 \mu-b) \alpha_{0}+\alpha_{2}\right) e^{1} \wedge e^{2}+\left(\alpha_{0}-b \alpha_{2}\right) e^{0} \wedge e^{1} \\
=s\left(\alpha_{0} e^{1} \wedge e^{2}+\alpha_{1} e^{0} \wedge e^{2}-\alpha_{2} e^{0} \wedge e^{1}\right) .
\end{gathered}
$$

We distinguish the cases $\alpha_{2}=0$ and $\alpha_{2} \neq 0$. If $\alpha_{2}=0$ then we must have $\alpha_{0}=0$ and $\alpha_{1}^{2}=1$ and $a=s$ is the unique solution. If $\alpha_{2} \neq 0$ and $b=s$ then $\alpha_{0}=0$ and $\alpha_{2}=0$, a contradiction. Assume then that $\alpha_{2} \neq 0$ and $b \neq s$. It follows that $\alpha_{0} \neq 0$ and

$$
b^{2}-2 b(s+\mu)+2(s \mu+1)=0 .
$$

The previous equation has the unique solution $b=\mu+s$, which indeed satisfies $b \neq s$. Then, the solutions in this case are given by:

$$
\alpha_{1}^{2}=1, \quad a=s, \quad \alpha_{0}=\mu \alpha_{2} .
$$


To classify which para-contact structures are also $\varepsilon \eta$-Einstein, we proceed as in the previous cases by direct computation. We obtain (imposing $a=s$ ):

$$
\begin{gathered}
\operatorname{Ric}^{g}\left(e_{0}, e_{0}\right)=\frac{(2 \mu-b)^{2}}{2}-\frac{(b-s)^{2}}{2}, \quad \operatorname{Ric}^{g}\left(e_{0}, e_{1}\right)=0, \\
\operatorname{Ric}^{g}\left(e_{1}, e_{1}\right)=-\frac{1}{2}, \quad \operatorname{Ric}^{g}\left(e_{0}, e_{2}\right)=s+2(\mu-b), \\
\operatorname{Ric}^{g}\left(e_{2}, e_{2}\right)=\frac{(2 \mu-b)^{2}}{2}+\frac{1}{2}+s(2 \mu-b)-\frac{b^{2}}{2}, \quad \operatorname{Ric}^{g}\left(e_{1}, e_{2}\right)=0 .
\end{gathered}
$$

We distinguish again between the cases $\alpha_{2}=0$ and $\alpha_{2} \neq 0$ with $b \neq s$.

(1) If $\alpha_{2}=0$ then $\alpha_{0}=0$ and $a=s$. The only non-trivial off-diagonal component of the $\varepsilon \eta$ Einstein condition is $b=\mu+\frac{s}{2}$. Imposing this condition in the time-like diagonal component we obtain

$$
\operatorname{Ric}^{g}\left(e_{0}, e_{0}\right)=0=\frac{1}{2}\left(\lambda^{2}+\kappa\right)
$$

whence $\kappa=-\lambda^{2}$. Since $\kappa \geq 0$ we conclude that $\lambda=\kappa=0$. However, this is incompatible with $\operatorname{Ric}^{g}\left(e_{1}, e_{1}\right)=-\frac{1}{2}$.

(2) If $\alpha_{2} \neq 0$ and $b=\mu+s$ then equation $\operatorname{Ric}^{g}\left(e_{1}, e_{2}\right)=0$ implies $\kappa=0$ since $\alpha_{1} \neq 0$ and $\alpha_{2} \neq 0$. However, then we would need $\operatorname{Ric}^{g}\left(e_{0}, e_{2}\right)=-s$ to vanish, what is not possible.

- Case $\mathfrak{g}_{5}$. In the orthonormal frame given in Appendix A, Equation $* \alpha=-s \mathrm{~d} \alpha$ is equivalent to

$$
\left(a \alpha_{1}+b \alpha_{2}\right) e^{0} \wedge e^{1}+\left(c \alpha_{1}+d \alpha_{2}\right) e^{0} \wedge e^{2}=s\left(\alpha_{0} e^{1} \wedge e^{2}+\alpha_{1} e^{0} \wedge e^{2}-\alpha_{2} e^{0} \wedge e^{1}\right),
$$

which immediately implies $\alpha_{0}=0$. The conditions for a para-contact structure to exist are $\alpha_{1}^{2}+\alpha_{2}^{2}=1$ and $a d=(b+s)(c-s)$, together with the conditions on the coefficients required by the algebra type $\mathfrak{g}_{5}$, which are $a c+b d=0$ and $a+d \neq 0$.

We verify now which para-contact structures on $\mathfrak{g}_{5}$ are $\varepsilon \eta$-Einstein. Using Appendix B we obtain the following components for the Ricci tensor:

$$
\begin{gathered}
\operatorname{Ric}^{g}\left(e_{0}, e_{0}\right)=-a^{2}-d^{2}-c b-\frac{b^{2}}{2}-\frac{c^{2}}{2}, \quad \operatorname{Ric}^{g}\left(e_{0}, e_{1}\right)=0, \\
\operatorname{Ric}^{g}\left(e_{1}, e_{1}\right)=a^{2}+a d-\frac{c^{2}}{2}+\frac{b^{2}}{2}, \quad \operatorname{Ric}^{g}\left(e_{0}, e_{2}\right)=0, \\
\operatorname{Ric}^{g}\left(e_{2}, e_{2}\right)=d^{2}+d a+\frac{c^{2}}{2}-\frac{b^{2}}{2}, \quad \operatorname{Ric}^{g}\left(e_{1}, e_{2}\right)=a c+b d .
\end{gathered}
$$

We have, $\operatorname{Ric}^{g}\left(e_{1}, e_{2}\right)=0$ automatically by the definition of algebra of type $\mathfrak{g}_{5}$. Hence, the $\varepsilon \eta$-Einstein condition evaluated in $e_{1}$ and $e_{2}$ implies:

$$
\alpha_{1} \alpha_{2} \kappa=0 \text {. }
$$

On the other hand, since $\alpha_{1}^{2}+\alpha_{2}^{2}=1$, the $\varepsilon \eta$-Einstein equation implies:

$$
\operatorname{Ric}^{g}\left(e_{1}, e_{1}\right)+\operatorname{Ric}^{g}\left(e_{2}, e_{2}\right)=(a+d)^{2}=-\lambda^{2} .
$$

Since $a+d \neq 0$ by the coefficient conditions of the algebra of type $\mathfrak{g}_{5}$, the previous equation admits no solutions and therefore an algebra of type $\mathfrak{g}_{5}$ does not admit $\varepsilon \eta$-Einstein para-contact structures.

- Case $\mathfrak{g}_{6}$. In the orthonormal frame given in Appendix A, Equation $* \alpha=-s \mathrm{~d} \alpha$ is equivalent to

$$
\left(d \alpha_{0}+c \alpha_{2}\right) e^{0} \wedge e^{1}+\left(-b \alpha_{0}-a \alpha_{2}\right) e^{1} \wedge e^{2}=s\left(\alpha_{0} e^{1} \wedge e^{2}+\alpha_{1} e^{0} \wedge e^{2}-\alpha_{2} e^{0} \wedge e^{1}\right),
$$

which immediately implies $\alpha_{1}=0$. From the previous (linear) equations we obtain that the conditions to have a non-trivial para-contact structures are $-\alpha_{0}^{2}+\alpha_{2}^{2}=1$ and $a d=(b+s)(c+s)$, 
together with the conditions $a c-b d=0$ and $a+d \neq 0$ required by the algebra type $\mathfrak{g}_{6}$. The components of the Ricci tensor read:

$$
\begin{gathered}
\operatorname{Ric}^{g}\left(e_{0}, e_{0}\right)=d^{2}+a d+\frac{b^{2}}{2}-\frac{c^{2}}{2}, \quad \operatorname{Ric}^{g}\left(e_{0}, e_{1}\right)=0, \\
\operatorname{Ric}^{g}\left(e_{1}, e_{1}\right)=-a^{2}-d^{2}-b c+\frac{c^{2}}{2}+\frac{b^{2}}{2}, \quad \operatorname{Ric}^{g}\left(e_{0}, e_{2}\right)=-a c+b d=0, \\
\operatorname{Ric}^{g}\left(e_{2}, e_{2}\right)=-a^{2}-d a-\frac{c^{2}}{2}+\frac{b^{2}}{2}, \quad \operatorname{Ric}^{g}\left(e_{1}, e_{2}\right)=0 .
\end{gathered}
$$

We have $\operatorname{Ric}^{g}\left(e_{0}, e_{2}\right)=0$ identically by the conditions on the coefficients of an algebra of type $\mathfrak{g}_{6}$. Evaluating the $\varepsilon \eta$-Einstein condition on $e_{0}$ and $e_{2}$ we obtain

$$
\kappa \alpha_{0} \alpha_{2}=0 \text {. }
$$

Hence, either $\alpha_{0}=0$ or $\kappa=0$ since $\alpha_{2}=0$ is not allowed by the para-contact condition. If $\alpha_{0}=0$ then $\alpha_{2}^{2}=1$, which implies $a=0$ and $c=-s$. Taking into account that $a c-b d=0$ and $a+d \neq 0$, we further obtain that $b=0$, which in turn implies:

$$
\lambda^{2}=d^{2}=\kappa+1,
$$

whence $d^{2} \geq 1$ since we must have $\kappa \geq 0$. Altogether these conditions solve the $\varepsilon \eta$-Einstein equations of a para-contact structure on $\mathfrak{g}_{6}$. On other hand, if $\kappa=0$, a combination of the $\varepsilon \eta$-Einstein equations implies:

$$
a d=(b+s)(c+s), \quad a c-b d=0, \quad a^{2}-d^{2}=b^{2}-c^{2}, \quad(a-d)^{2}=(b-c)^{2} .
$$

The last equation above reduces to

$$
(c-b)=\mu(a-d)
$$

where $\mu \in \mathbb{Z}_{2}$. We distinguish now two cases:

(1) If $a=d \neq 0$ (using that $a+d \neq 0$ ) we obtain $c=b$ and the $\varepsilon \eta$-Einstein equations are equivalent to

$$
a^{2}=\frac{\lambda^{2}}{4}
$$

Likewise, the coefficient conditions required by the algebra of type $\mathfrak{g}_{6}$ are

$$
a^{2}=(b+s)^{2},
$$

implying $a=\sigma(b+s)$ for a sign $\sigma \in \mathbb{Z}_{2}$. Note that $b \neq s$ since $a+d=2 a \neq 0$. Plugging $a=\sigma(b+s)$ in Equation (15) we obtain:

$$
\alpha_{0}^{2}=\alpha_{2}^{2},
$$

which is incompatible with the para-contact condition $-\alpha_{0}^{2}+\alpha_{2}^{2}=1$.

(2) If $a \neq d$, equation $a^{2}-d^{2}=b^{2}-c^{2}$ is equivalent to

$$
a+d=-\mu(b+c) \text {. }
$$

Combining this equation with $(c-b)=\mu(a-d)$ we obtain $a=-\mu b$ and $d=-\mu c$. The constraints on the coefficients required by the algebra of type $\mathfrak{g}_{6}$ are found to reduce to

$$
1+s(b+c)=0,
$$

whereas the $\varepsilon \eta$-Einstein equations are tantamount to

$$
\lambda^{2}=(b+c)^{2} .
$$

Therefore, using that $1+s(b+c)=0$ we obtain $\lambda^{2}=1$. Equation (15) is solved by:

$$
b=-\mu a, \quad c=-s+\mu a, \quad d=\mu s-a, \quad(-s+\mu a) \alpha_{0}=a \alpha_{2}, \quad \alpha_{2}^{2}=1+\alpha_{0}^{2} .
$$


- Case $\mathfrak{g}_{7}$. In the orthonormal frame given in Appendix A, Equation $* \alpha=-s \mathrm{~d} \alpha$ is equivalent to

$$
\begin{aligned}
\left(b \alpha_{0}+a \alpha_{1}+b \alpha_{2}\right) e^{0} \wedge e^{1} & +\left(d \alpha_{0}+c \alpha_{1}+d \alpha_{2}\right) e^{0} \wedge e^{2}+\left(b \alpha_{0}+a \alpha_{1}+b \alpha_{2}\right) e^{1} \wedge e^{2} \\
& =s\left(\alpha_{0} e^{1} \wedge e^{2}+\alpha_{1} e^{0} \wedge e^{2}-\alpha_{2} e^{0} \wedge e^{1}\right) .
\end{aligned}
$$

which immediately implies $\alpha_{0}+\alpha_{2}=0$, whence $\alpha_{1}^{2}=1$. Write $\alpha_{1}=\sigma$, with $\sigma \in \mathbb{Z}_{2}$. With these assumptions, the previous equations boil down to

$$
\alpha_{0}=s \sigma a, \quad c=s, \quad \alpha_{2}^{2}=a^{2},
$$

where $\mu \in \mathbb{Z}_{2}$. Since $c \neq 0$, then $a=0$ from the condition $a c=0$ required by the algebra of type $\mathfrak{g}_{7}$. Hence $\alpha_{0}=\alpha_{2}=0$. With these provisos in mind, the Ricci curvature reads:

$$
\begin{gathered}
\operatorname{Ric}^{g}\left(e_{0}, e_{0}\right)=-b s-\frac{1}{2}, \quad \operatorname{Ric}^{g}\left(e_{0}, e_{1}\right)=0, \\
\operatorname{Ric}^{g}\left(e_{1}, e_{1}\right)=-\frac{1}{2}, \quad \operatorname{Ric}^{g}\left(e_{0}, e_{2}\right)=s b, \\
\operatorname{Ric}^{g}\left(e_{2}, e_{2}\right)=\frac{1}{2}-s b, \quad \operatorname{Ric}^{g}\left(e_{1}, e_{2}\right)=0 .
\end{gathered}
$$

However, since $\alpha=\sigma e^{1}$, we obtain $b=0$, which implies $\kappa=-1$, a value that is not permitted by Definition 4.1 .

Finally, to verify which of the $\varepsilon \eta$-Einstein para-contact structures are Sasakian we apply first Proposition 2.19, which states that $\varepsilon \eta$-Einstein para-contact structures on $\widetilde{E}(1,1)$ or $\widetilde{E}(2)$ can never be Sasakian. Also, a direct computation shows that $\mathfrak{h}$ vanishes for every $\varepsilon \eta$-Einstein para-contact structure on $\widetilde{\mathrm{Sl}}(2, \mathbb{R})$ and $\mathfrak{g}_{6}$.

4.4. Lorentzian case with null Reeb vector field. When $\varepsilon=0$ the $\varepsilon \eta$-Einstein condition for an $\varepsilon$-contact metric structure $(\chi, \alpha)$ reduces to

$$
\operatorname{Ric}^{\chi}=-\frac{\lambda^{2}}{2} \chi+\kappa \alpha \otimes \alpha
$$

with $\kappa \geq 0$.

Remark 4.22. To the best of our knowledge, this equation has not been considered in the literature. In particular, the methods and techniques used in [11,38] to classify $(\kappa, \mu)$ and $\varepsilon \eta$-Einstein contact three manifolds do not seem to apply in this case, due to the fact that $\phi^{2}$ is not an isomorphism when restricted to the kernel of $\alpha$ and that $\mathfrak{h}$ cannot have non-zero eigenvalues, see Remarks 2.15 and 3.5.

The goal of this subsection is to classify all left-invariant $\varepsilon \eta$-Einstein null contact structures on a simply connected three-dimensional Lie group G. In order to do this, we will make use of the following lemma.

Lemma 4.23. Let $(\chi, \alpha)$ be a null contact metric structure and let $\{\xi, u, \phi(u)\}$ be a light-cone frame. Then $(\xi, \alpha)$ is $\varepsilon \eta$-Einstein if and only if

$$
\begin{gathered}
\operatorname{Ric}^{\chi}(\xi, \xi)=\operatorname{Ric}^{\chi}(\xi, \phi(u))=\operatorname{Ric}^{\chi}(u, \phi(u))=0, \\
\operatorname{Ric}^{\chi}(\xi, u)=\operatorname{Ric}^{\chi}(\phi(u), \phi(u))=-\frac{\lambda^{2}}{2}, \quad \operatorname{Ric}^{\chi}(u, u)=\kappa .
\end{gathered}
$$

Proof. Follows by direct computation.

Theorem 4.24. A three-dimensional connected and simply connected Lie group $\mathrm{G}$ admits a leftinvariant $\varepsilon \eta$-Einstein null contact structure $(g, \alpha)$ if and only if $(\mathrm{G}, g, \alpha)$ is isomorphic, through a possibly orientation-reversing isometry, to one of the items listed in the following table in terms of the orthonormal frame $\left\{e_{0}, e_{1}, e_{2}\right\}$ appearing in Theorem A.1: 


\begin{tabular}{|c|c|c|c|c|c|}
\hline $\mathfrak{g}$ & Structure constants $\left(s \in \mathbb{Z}_{2}\right)$ & $\alpha$ & $\eta$-Einstein constants & $\mathrm{G}$ & Sasakian \\
\hline \multirow{4}{*}{$\mathfrak{g}_{3}$} & $a=b=c=s$ & $\alpha_{0}^{2}=\alpha_{1}^{2}+\alpha_{2}^{2}$ & $\lambda^{2}=1, \kappa=0$ & $\widetilde{\mathrm{S}}(2, \mathbb{R})$ & Yes \\
\cline { 2 - 6 } & $a=c=s, b=0$ & $\alpha_{2}=0$, & $\lambda^{2}=0, \kappa=0$ & $\widetilde{\mathrm{E}}(1,1)$ & No \\
& & $\alpha_{0}^{2}=\alpha_{1}^{2}$ & & & \\
\hline \multirow{2}{*}{$\mathfrak{g}_{4}$} & $b=0, a=s$ & $\alpha_{1}=0$, & $\lambda^{2}=1, \alpha_{0}^{2} \kappa=1$ & $\widetilde{\mathrm{Sl}}(2, \mathbb{R})$ & Yes \\
\cline { 2 - 5 } & $b=0, a=0$, & $\alpha_{0}=\mu \alpha_{2}$ & $\lambda^{2}=0, \alpha_{0}^{2} \kappa=2$ & $\widetilde{\mathrm{E}}(1,1)$ & No \\
\hline \multirow{2}{*}{$\mathfrak{g}_{6}$} & $a=d \neq 0, b=c$ & $\alpha_{1}=0$, & $\lambda^{2}=4 a^{2}$, & $\mathfrak{G}_{6}$ & \multirow{2}{*}{ If $a=\frac{\mu s}{2}$} \\
& $a=\mu(b+s), \mu \in \mathbb{Z}_{2}$ & $\alpha_{0}=-\mu \alpha_{2}$ & $\kappa=0$ & & \\
\hline
\end{tabular}

Proof. Proposition 3.11 classifies all simply-connected Lorentzian Lie groups admitting left-invariant null contact structures. Hence, we will proceed by verifying which of the cases appearing in Proposition 3.11 satisfy the $\varepsilon \eta$-Einstein equation. For this, we will use the formulae presented in Appendix $\mathrm{B}$, where the Ricci tensor is computed on a global orthonormal frame.

- Case $\mathfrak{g}_{1}$. The Reeb vector is given by $\xi=-\alpha_{0}\left(e_{0}+e_{2}\right)$. A direct computation using Appendix B shows that $\operatorname{Ric}^{\chi}(u, \phi(u))=-\frac{s a}{\alpha_{0}}$. Since $a \neq 0$ by the definition of algebra of type $\mathfrak{g}_{1}$, Lemma 4.23 implies that $\mathfrak{g}_{1}$ does not admit null $\varepsilon \eta$-Einstein structures.

- Case $\mathfrak{g}_{3}$. We distinguish between the cases $\alpha_{1}=0, \alpha_{2}=0$ and $\alpha_{1}, \alpha_{2} \neq 0$. If $\alpha_{1}=0$, a light-cone frame is given by $\xi=\alpha_{0}\left(-e_{0}+\mu e_{2}\right), u=\frac{1}{2 \alpha_{0}}\left(e_{0}+\mu e_{2}\right)$ and $\phi(u)=\mu e_{1}$. We obtain:

$$
\begin{gathered}
\operatorname{Ric}^{\chi}(\xi, \xi)=\operatorname{Ric}^{\chi}(\xi, \phi(u))=\operatorname{Ric}^{\chi}(u, \phi(u))=0 \\
\operatorname{Ric}^{\chi}(\xi, u)=-a+\frac{a^{2}}{2}, \quad \operatorname{Ric}^{\chi}(\phi(u), \phi(u))=-\frac{a^{2}}{2}, \quad \operatorname{Ric}^{\chi}(u, u)=0 .
\end{gathered}
$$

Hence, the $\varepsilon \eta$-Einstein implies $a=0$ or $a=s$. Since $a=0$ is not allowed, we conclude $a=s$, which in turn implies $\lambda^{2}=1$ and $\kappa=0$.

If $\alpha_{2}=0$, a similar analysis follows. In this case, a light-cone frame is given by $\xi=\alpha_{0}\left(-e_{0}+\right.$ $\left.\mu e_{1}\right), u=\frac{1}{2 \alpha_{0}}\left(e_{0}+\mu e_{1}\right)$ and $\phi(u)=-\mu e_{2}$. We obtain:

$$
\begin{gathered}
\operatorname{Ric}^{\chi}(\xi, \xi)=\operatorname{Ric}^{\chi}(\xi, \phi(u))=\operatorname{Ric}^{\chi}(u, \phi(u))=0 \\
\operatorname{Ric}^{\chi}(\xi, u)=-b s+\frac{b^{2}}{2}, \quad \operatorname{Ric}^{\chi}(\phi(u), \phi(u))=-\frac{b^{2}}{2}, \quad \operatorname{Ric}^{\chi}(u, u)=0,
\end{gathered}
$$

which implies either $b=s$ or $b=0$. For $b=s, \lambda^{2}=1$ and $\kappa=0$, and for $b=0, \lambda^{2}=\kappa=0$.

Finally, if $\alpha_{1}, \alpha_{2} \neq 0$, we directly obtain $\operatorname{Ric}^{\chi}=-\frac{1}{2} \chi$ and every null contact structure of this type is $\varepsilon \eta$-Einstein (with $\lambda^{2}=1$ and $\kappa=0$ ).

- Case $\mathfrak{g}_{4}$. We choose a light-cone frame $\{\xi, u, \phi(u)\}$ with $\xi=\alpha_{0}\left(-e_{0}+\mu e_{2}\right), u=\frac{1}{2 \alpha_{0}}\left(e_{0}+\mu e_{2}\right)$ and $\phi(u)=\mu e_{1}$. We compute:

$$
\begin{gathered}
\operatorname{Ric}^{\chi}(\xi, \xi)=\operatorname{Ric}^{\chi}(\xi, \phi(u))=\operatorname{Ric}^{\chi}(u, \phi(u))=0 \\
\operatorname{Ric}^{\chi}(\xi, u)=\frac{a^{2}}{2}-a s, \quad \operatorname{Ric}^{\chi}(\phi(u), \phi(u))=-\frac{a^{2}}{2}, \quad \operatorname{Ric}^{\chi}(u, u)=\frac{\mu}{\alpha_{0}^{2}}(a-2 s) .
\end{gathered}
$$

These conditions are satisfied if and only if $a=0$ or $a=s$. For $a=s, \lambda^{2}=1$ and $\kappa=-\frac{s \mu}{\alpha_{0}^{2}}$, and for $a=0, \lambda^{2}=0$ and $\kappa=-2 \frac{s \mu}{\alpha_{0}^{2}}$. Since $\kappa$ must be non-negative by definition, we must require that $s \mu=-1$, which implies that $b=0$. 
- Case $\mathfrak{g}_{6}$. We can choose the light-cone frame $\{\xi, u, \phi(u)\}$ with $\xi=-\alpha_{0}\left(e_{0}+\mu e_{2}\right), u=\frac{1}{2 \alpha_{0}}\left(e_{0}-\right.$ $\left.\mu e_{2}\right)$ and $\phi(u)=-\mu e_{1}$. Imposing the constraints found in Proposition 3.11 for null contact structures on $\mathfrak{g}_{6}$, we get the following components for the Ricci curvature:

$$
\begin{gathered}
\operatorname{Ric}^{\chi}(\xi, \phi(u))=\operatorname{Ric}^{\chi}(u, \phi(u))=0, \quad \operatorname{Ric}^{\chi}(\xi, \xi)=0, \\
\operatorname{Ric}^{\chi}(\xi, u)=-2 a^{2}, \quad \operatorname{Ric}^{\chi}(\phi(u), \phi(u))=-2 a^{2}, \quad \operatorname{Ric}^{\chi}(u, u)=0 .
\end{gathered}
$$

These equations yield an $\varepsilon \eta$-Einstein structure with $\lambda^{2}=4 a^{2} \neq 0$ and $\kappa=0$.

Finally, in order to determine when the different $\varepsilon \eta$-Einstein structures obtained are Sasakian, we just have to make use of Proposition 3.16.

\section{SiX-Dimensional SUPERGRAVITY AND $\varepsilon$-CONTACT STRUCTURES}

In the following, let $M$ be an oriented and spin six-dimensional manifold.

Definition 5.1. The bosonic configuration space of six-dimensional minimal supergravity coupled to a tensor multiplet with constant dilaton on $M$ is defined as the following set:

$$
\operatorname{Conf}(M) \stackrel{\text { def. }}{=}\left\{(\mathrm{g}, \mathrm{H}) \in \operatorname{Lor}(M) \times \Omega^{3}(M)\right\},
$$

where $\operatorname{Lor}(M)$ denotes the set of Lorentzian metrics on $M$.

Given $(\mathrm{g}, \mathrm{H}) \in \operatorname{Conf}(M)$ we define $\nabla^{\mathrm{H}}$ to be the unique metric-compatible connection on $(M, \mathrm{~g})$ with totally skew-symmetric torsion given by $\mathrm{H} \in \Omega^{3}(M)$. In more explicit terms we have

$$
\nabla^{\mathrm{H}}=\nabla+\frac{1}{2} \mathrm{~g}^{-1} \mathrm{H}
$$

where $\nabla$ denotes the Levi-Civita connection associated to g.

Definition 5.2. A pair $(\mathrm{g}, \mathrm{H}) \in \operatorname{Conf}(M)$ is a bosonic solution of six-dimensional minimal supergravity coupled to a tensor multiplet with constant dilaton on $M$ if:

$$
\operatorname{Ric}\left(\nabla^{\mathrm{H}}\right)=0, \quad \mathrm{dH}=0, \quad \mathrm{~d} *_{\mathrm{g}} \mathrm{H}=0, \quad|\mathrm{H}|_{\mathrm{g}}^{2}=0,
$$

where $\operatorname{Ric}\left(\nabla^{\mathrm{H}}\right) \in \Gamma\left(\operatorname{Sym}^{2}\left(T^{*} M\right)\right)$ is the Ricci curvature tensor of $\nabla^{\mathrm{H}}$ and $*_{\mathrm{g}}: \Omega^{3}(M) \rightarrow \Omega^{3}(M)$ denotes the Hodge dual associated to g. We denote by $\operatorname{Sol}(M) \subset \operatorname{Conf}(M)$ the set of solutions on $M$.

Remark 5.3. In six Lorentzian dimensions the Hodge dual $*_{\mathrm{g}}$ on three-forms squares to the identity. Hence, we obtain the splitting

$$
\Lambda^{3}(M)=\Lambda_{+}^{3}(M) \oplus \Lambda_{-}^{3}(M),
$$

in terms of self dual $\Lambda_{+}^{3}(M)$ and anti-self dual $\Lambda_{-}^{3}(M)$ three-forms. Using this decomposition, a particular class of solutions of Equations (16) is obtained by requiring $\mathrm{H}$ to be self-dual, that is:

$$
\operatorname{Ric}\left(\nabla^{\mathrm{H}}\right)=0, \quad \mathrm{dH}=0, \quad *_{\mathrm{g}} \mathrm{H}=\mathrm{H},
$$

This set of equations define the bosonic equations of six-dimensional minimal supergravity. Equations (16) are more general and allow, for instance, $\mathrm{H}$ to be a section of a fixed lagrangian distribution of $\Lambda^{3}(M)$. The possibility of generalizing minimal supergravity to this situation was proposed in [32] and it remains, to the best of our knowledge, up for debate. 
Theorem 5.4. Let:

$$
\left(N, \chi, \alpha_{N}, \varepsilon_{N}\right) \in \operatorname{PCont}_{L}^{\varepsilon \eta}\left(\varepsilon_{N}, \lambda^{2}, \kappa_{N}=l^{2}\right), \quad\left(X, h, \alpha_{X}\right) \in \operatorname{PCont}_{R}^{\varepsilon \eta}\left(\lambda^{2}, \kappa_{X}=\left|\alpha_{N}\right|^{2} l^{2}\right) .
$$

Then, the oriented Cartesian product manifold

$$
M=N \times X
$$

carries a family of solutions $\left(\mathrm{g}, \mathrm{H}_{\lambda, l}\right) \in \operatorname{Sol}(M)$ of six-dimensional minimal supergravity coupled to a tensor multiplet with constant dilaton given by

$$
\mathrm{g}=\chi \oplus h, \quad \mathrm{H}_{\lambda, l}=\lambda \nu_{\chi}+\frac{l}{3}\left(*_{\chi} \alpha_{N}\right) \wedge \alpha_{X}+\frac{l}{3} \alpha_{N} \wedge\left(*_{h} \alpha_{X}\right)+\lambda \nu_{h}
$$

and parametrized by $(\lambda, l) \in \mathbb{R}^{2}$. Equivalently, the oriented Cartesian Lorentzian product of $\left(N, \chi, \alpha_{N}, \varepsilon_{N}\right) \in \operatorname{PCont}_{L}^{\varepsilon \eta}\left(\varepsilon_{N}, \lambda, l\right)$ and $\left(X, h, \alpha_{X}\right) \in \operatorname{PCont}_{R}^{\varepsilon \eta}\left(\lambda, \kappa=\varepsilon_{N}, l\right)$ carries a bi-parametric family of metric-compatible, Ricci-flat, connections with totally skew-symmetric, isotropic, closed and co-closed torsion prescribed by $\mathrm{H}_{\lambda, l}$.

Proof. We first compute that $\mathrm{H}_{\lambda, l}$, as prescribed in the statement of the theorem, is closed:

$$
\begin{aligned}
& \mathrm{dH}_{\lambda, l}=\frac{l}{3} \mathrm{~d}\left(*_{\chi} \alpha_{N}\right) \wedge \alpha_{X}+\frac{l}{3}\left(*_{\chi} \alpha_{N}\right) \wedge \mathrm{d} \alpha_{X}+\frac{l}{3} \mathrm{~d} \alpha_{N} \wedge\left(*_{h} \alpha_{X}\right)-\frac{l}{3} \alpha_{N} \wedge \mathrm{d}\left(*_{h} \alpha_{X}\right) \\
& =\frac{l}{3}\left(*_{\chi} \alpha_{N}\right) \wedge \mathrm{d} \alpha_{X}+\frac{l}{3} \mathrm{~d} \alpha_{N} \wedge\left(*_{h} \alpha_{X}\right)=\frac{l}{3} *_{\chi} \alpha_{N} \wedge *_{h} \alpha_{X}-\frac{l}{3} *_{\chi} \alpha_{N} \wedge *_{h} \alpha_{X}=0,
\end{aligned}
$$

where we have used that $\mathrm{d} \alpha_{N}=-*_{\chi} \alpha_{N}$ and $\mathrm{d} \alpha_{X}=*_{h} \alpha_{X}$ by the $\varepsilon$-contact condition. In addition, $\mathrm{H}_{\lambda, l}$ is co-closed:

$$
\begin{aligned}
\mathrm{d} * \mathrm{H}_{\lambda, l} & =\lambda \mathrm{d} * \nu_{\chi}+\frac{l}{3} \mathrm{~d} *\left(*_{\chi} \alpha_{N} \wedge \alpha_{X}\right)+\frac{l}{3} \mathrm{~d} *\left(\alpha_{N} \wedge *_{h} \alpha_{X}\right)+\lambda \mathrm{d} * \nu_{h} \\
& =-\lambda \mathrm{d} \nu_{h}+\frac{l}{3} \mathrm{~d}\left(\alpha_{N} \wedge *_{h} \alpha_{X}\right)+\frac{l}{3} \mathrm{~d}\left(*_{\chi} \alpha_{N} \wedge \alpha_{X}\right)-\lambda \mathrm{d} \nu_{\chi}=0
\end{aligned}
$$

where we have used again the $\varepsilon$-contact condition and the fact that, for $\rho \in \Omega^{q}(N)$ and $\sigma \in \Omega^{r}(X)$, we have $*(\rho \wedge \sigma)=(-1)^{r(3-q)} *_{\chi} \rho \wedge *_{h} \sigma$. We verify now the norm of $\mathrm{H}_{\lambda, l}$ indeed vanishes:

$$
\begin{gathered}
\left|\mathrm{H}_{\lambda, l}\right|_{\mathrm{g}}^{2}=\lambda^{2} \mathrm{~g}\left(\nu_{\chi}, \nu_{\chi}\right)+\frac{l^{2}}{9} \mathrm{~g}\left(\left(*_{\chi} \alpha_{N}\right) \wedge \alpha_{X},\left(*_{\chi} \alpha_{N}\right) \wedge \alpha_{X}\right)+\frac{l^{2}}{9} \mathrm{~g}\left(\alpha_{N} \wedge\left(*_{h} \alpha_{X}\right), \alpha_{N} \wedge\left(*_{h} \alpha_{X}\right)\right) \\
+\lambda^{2} \mathrm{~g}\left(\nu_{h}, \nu_{h}\right)=\lambda^{2} \chi\left(\nu_{\chi}, \nu_{\chi}\right)+\frac{l^{2}}{3} \chi\left(*_{\chi} \alpha_{N}, *_{\chi} \alpha_{N}\right) h\left(\alpha_{X}, \alpha_{X}\right)+\frac{l^{2}}{3} \chi\left(\alpha_{N}, \alpha_{N}\right) h\left(*_{h} \alpha_{X}, *_{h} \alpha_{X}\right) \\
+\lambda^{2} h\left(\nu_{h}, \nu_{h}\right)=-6 \lambda^{2}-\frac{2 l^{2}}{3} \chi\left(\alpha_{N}, \alpha_{N}\right) h\left(\alpha_{X}, \alpha_{X}\right)+\frac{2 l^{2}}{3} \chi\left(\alpha_{N}, \alpha_{N}\right) h\left(\alpha_{X}, \alpha_{X}\right)+6 \lambda^{2}=0 .
\end{gathered}
$$

We verify next that the Einstein equations are satisfied for the specific choices of constants specified in the statement. We have that

$$
\begin{gathered}
\left.\mathrm{H}_{\lambda, l} \circ \mathrm{H}_{\lambda, l}\right|_{T N \otimes} T N=\lambda^{2} \nu_{N} \circ \nu_{N}+\left.\frac{l^{2}}{9}\left(*_{\chi} \alpha_{N} \wedge \alpha_{X}\right) \circ\left(*_{\chi} \alpha_{N} \wedge \alpha_{X}\right)\right|_{T N \otimes T N} \\
+\left.\frac{l^{2}}{9}\left(\alpha_{N} \wedge *_{h} \alpha_{X}\right) \circ\left(\alpha_{N} \wedge *_{h} \alpha_{X}\right)\right|_{T N \otimes T N} .
\end{gathered}
$$

We compute also the following:

$$
\begin{gathered}
\lambda^{2} \nu_{N} \circ \nu_{N}=-2 \lambda^{2} \chi,\left.\quad\left(\alpha_{N} \wedge *_{h} \alpha_{X}\right) \circ\left(\alpha_{N} \wedge *_{h} \alpha_{X}\right)\right|_{T N \otimes T N}=18 \alpha_{N} \otimes \alpha_{N} \\
\left.\left(*_{\chi} \alpha_{N} \wedge \alpha_{X}\right) \circ\left(*_{\chi} \alpha_{N} \wedge \alpha_{X}\right)\right|_{T N \otimes T N}=18\left(\alpha_{N} \otimes \alpha_{N}-\left|\alpha_{N}\right|_{\chi}^{2} \chi\right) .
\end{gathered}
$$

This implies that

$$
\left.\frac{1}{4} \mathrm{H}_{\lambda, l} \circ \mathrm{H}_{\lambda, l}\right|_{T N \otimes T N}=-\frac{\lambda^{2}}{2} \chi-\frac{l^{2}}{2}\left|\alpha_{N}\right|_{\chi}^{2} \chi+l^{2} \alpha_{N} \otimes \alpha_{N} .
$$


Likewise we have

$$
\begin{gathered}
\left.\mathrm{H}_{\lambda, l} \circ \mathrm{H}_{\lambda, l}\right|_{T X \otimes T X}=\lambda^{2} \nu_{X} \circ \nu_{X}+\left.\frac{l^{2}}{9}\left(*_{\chi} \alpha_{N} \wedge \alpha_{X}\right) \circ\left(*_{\chi} \alpha_{N} \wedge \alpha_{X}\right)\right|_{T X \otimes T X} \\
+\left.\frac{l^{2}}{9}\left(\alpha_{N} \wedge *_{h} \alpha_{X}\right) \circ\left(\alpha_{N} \wedge *_{h} \alpha_{X}\right)\right|_{T X \otimes T X}
\end{gathered}
$$

which in turn implies

$$
\left.\frac{1}{4} \mathrm{H}_{\lambda, l} \circ \mathrm{H}_{\lambda, l}\right|_{T X \otimes T X}=\frac{\lambda^{2}}{2} h+\frac{l^{2}}{2}\left|\alpha_{N}\right|_{\chi}^{2} h-l^{2}\left|\alpha_{N}\right|_{\chi}^{2} \alpha_{X} \otimes \alpha_{X} .
$$

Finally, it can be checked the mixed components vanish identically,

$$
\left.\mathrm{H}_{\lambda, l} \circ \mathrm{H}_{\lambda, l}\right|_{T N \otimes T X}=\left.\mathrm{H}_{\lambda, l} \circ \mathrm{H}_{\lambda, l}\right|_{T X \otimes T N}=0 .
$$

From Definition 4.1, we obtain that $\left(N, \chi, \alpha_{N}, \varepsilon_{N}\right) \in \operatorname{PCont}_{L}^{\varepsilon \eta}\left(\varepsilon_{N}, \lambda^{2}, \kappa_{N}=l^{2}\right)$ and $\left(M, h, \alpha_{X}\right) \in$ PCont $_{R}^{\varepsilon \eta}\left(\lambda^{2}, \kappa_{X}=\left|\alpha_{N}\right|^{2} l^{2}\right)$ satisfy, respectively:

$$
\operatorname{Ric}^{\chi}=-\frac{1}{2}\left(\lambda^{2}+l^{2} \varepsilon\right) \chi+l^{2} \alpha_{N} \otimes \alpha_{N}, \quad \operatorname{Ric}^{h}=\frac{1}{2}\left(\lambda^{2}+l^{2} \varepsilon\right) h-l^{2} \varepsilon \alpha_{X} \otimes \alpha_{X} .
$$

Since by definition $\varepsilon=\left|\alpha_{N}\right|_{\chi}^{2}$, the right hand sides of the equations appearing in (20) coincide with the right hand sides of equations (18) and (19), respectively. Hence, the tuple $\left(\mathrm{g}, \mathrm{H}_{\lambda, l}\right)$ as defined in the statement of the Theorem satisfies:

$$
\operatorname{Ric}\left(\nabla^{\mathrm{H}_{\lambda, l}}\right)=0, \quad \mathrm{dH}_{\lambda, l}=0, \quad \mathrm{~d} *_{\mathrm{g}} \mathrm{H}_{\lambda, l}=0, \quad\left|\mathrm{H}_{\lambda, l}\right|_{\mathrm{g}}^{2}=0,
$$

whence it is a solution of 6-dimensional supergravity coupled to a tensor multiplet with constant dilaton.

Remark 5.5. For ease of reference, we will refer to the solutions $(\mathrm{g}, \mathrm{H})$ constructed in Theorem 5.4 as $\varepsilon$-contact supergravity solutions of type $\left(\varepsilon_{N}, \lambda, l\right)$, where $\varepsilon_{N} \in\{-1,0,1\}$ is the norm of the Reeb vector field of the Lorentzian $\varepsilon$-contact structure occurring in the given solution.

The holonomy of the Levi-Civita connection $\nabla$ of an $\varepsilon$-contact supergravity solution $(\mathrm{g}, \mathrm{H})$ is clearly reducible, since the sub-bundles $T N \subset T M$ and $T X \subset T M$ are preserved by $\nabla$ by construction. In particular, $\nabla$ is a product connection on $T M=T N \times T X$. However, the connection with torsion $\nabla^{\mathrm{H}}$ is in general not a product connection. In particular neither $T N \subset T M$ nor $T X \subset T M$ are preserved by $\nabla^{\mathrm{H}}$ if $l \neq 0$. Therefore supergravity $\varepsilon$-contact solutions are in general not the direct product of a pair of three-dimensional pseudo-Riemannian manifolds with torsion.

Theorem 5.4 allows to construct large classes of explicit solutions of 6-dimensional supergravity coupled to a tensor multiplet with constant dilaton by exploiting the extensive literature on $\varepsilon \eta$-Einstein Riemannian and Lorentzian (para) contact metric three-manifolds, as discussed in Section 4, and by employing new null contact metric structures, as discussed in Sections 3 and 4. In particular, the previous Theorem implies that the construction of examples and development of classification results on Riemannian and Lorentzian $\varepsilon \eta$-Einstein (para) contact metric three-manifolds can automatically be used to construct new Lorentzian six-manifolds equipped with a Ricci-flat metric-compatible connection with totally skew-symmetric, isotropic, closed and co-closed torsion.

\section{RICCI FLAT LORENTZIAN SIX-MANIFOLDS WITH CLOSED SELF-DUAL TORSION}

In this section we apply the results of the previous sections to the construction of new sixdimensional Lorentzian manifolds equipped with a Ricci-flat and metric-compatible connection with totally skew-symmetric, isotropic, closed and co-closed torsion, which in turn yields new solutions of minimal supergravity coupled to a tensor multiplet with constant dilaton in six dimensions. 
Excluding the Ricci-flat case, the simplest scenario where Theorem 5.4 applies is obtained by taking $l=0$ and $\lambda \neq 0$. In this situation, the corresponding six-dimensional $\varepsilon$-contact solution is given by:

$$
\mathrm{g}=\chi \oplus h, \quad \mathrm{H}_{\lambda, l}=\lambda\left(\nu_{\chi}+\nu_{h}\right), \quad \lambda \neq 0,
$$

on $M=N \times X$, where $\chi$ and $h$ are Einstein with negative and positive Einstein constant, respectively. Assuming that both $(N, \chi)$ and $(X, h)$ are connected, simply connected and geodesically complete we conclude that $(X, h)$ is isometric to the round sphere and $(N, \chi)$ is isometric to $\widetilde{\mathrm{Sl}}(2, \mathbb{R})$ equipped with its Einstein metric. In particular:

$$
(M, g)=\left(\widetilde{\mathrm{Sl}}(2, \mathbb{R}) \times S^{3}, \chi \oplus h\right),
$$

is a solution of six-dimensional minimal supergravity, which corresponds with the well-known $\mathrm{AdS}_{3} \times S^{3}$ maximally supersymmetric solution of the theory [22]. For $l \neq 0, \varepsilon$-contact supergravity solutions are not isomorphic to the previous solution ${ }^{3}$. Hence, intuitively speaking we can think of $\varepsilon$-contact supergravity solutions with $l \neq 0$ as being generically non-supersymmetric geometric and topological deformations of the supersymmetric $\mathrm{AdS}_{3} \times S^{3}$ solution, with deformations parametrized by $l \in \mathbb{R}, \alpha_{N} \in \Omega^{1}(N)$ and $\alpha_{X} \in \Omega^{1}(X)$. In the following we consider $\varepsilon$-contact supergravity solutions of type $\varepsilon_{N}=-1, \varepsilon_{N}=0$ and $\varepsilon_{N}=1$ separately. We emphasise that in general the values of $\lambda^{2}$ and $\kappa$ do not uniquely determine the diffeomorphism type of $N$ or $X$ and that the same diffeomorphism type may admite several non-isomorphic $\varepsilon$-contact supergravity solutions. In this direction, it is a priori possible that there exist, at least when $\varepsilon_{N} \neq 1$ or in the Sasakian case for $\varepsilon_{N}=1, \varepsilon$-contact supergravity solutions for which $M$ is not diffeomorphic to a Lie group.

6.1. Time-like case: $\varepsilon_{N}=-1$. Let:

$$
\left(N, \chi, \alpha_{N},-1\right) \in \operatorname{PCont}_{L}^{\varepsilon \eta}\left(\lambda^{2}, l^{2},-1\right), \quad\left(X, g, \alpha_{X}\right) \in \operatorname{PCont}_{R}^{\varepsilon \eta}\left(\lambda^{2},-l^{2}\right) .
$$

\begin{tabular}{|c|c|c|c|c|}
\hline \multicolumn{2}{|c|}{ Lorentzian factor } & \multicolumn{2}{|c|}{ Riemannian factor } & \\
\hline$N$ & Sasakian & $X$ & Sasakian & $\left(\lambda^{2}, l^{2}\right)$ \\
\hline$\widetilde{\mathrm{Sl}}(2, \mathbb{R})$ & Yes & $\mathrm{SU}(2)$ & Yes & $\lambda^{2}=1-l^{2}, 1>l^{2} \geq 0$ \\
\hline $\mathfrak{G}_{6}$ & Yes & $\mathrm{SU}(2)$ & Yes & $\lambda^{2}=1-l^{2}, 1>l^{2} \geq 0$ \\
\hline $\mathrm{H}_{3}$ & Yes & $\mathrm{H}_{3}$ & Yes & $\lambda^{2}=0, l^{2}=1$ \\
\hline$\widetilde{\mathrm{Sl}}(2, \mathbb{R})$ & No & $\mathrm{SU}(2)$ & No & $\lambda^{2}=l^{2}, \frac{1}{2}>l^{2}>0$ \\
\hline$\widetilde{\mathrm{E}}(1,1)$ & No & $\widetilde{\mathrm{E}}(2)$ & No & $\lambda^{2}=0, l^{2}=0$ \\
\hline
\end{tabular}

Then, the product of the following pairs of three-manifolds carry $\varepsilon$-contact supergravity solutions as prescribed in Theorem 5.4 for the specified parameters:

6.2. Space-like case: $\varepsilon_{N}=1$. Let:

$$
\left(N, \chi, \alpha_{N}, 1\right) \in \mathrm{PCont}_{L}^{\varepsilon \eta}\left(\lambda^{2}, l^{2}, 1\right), \quad\left(X, g, \alpha_{X}\right) \in \operatorname{PCont}_{R}^{\varepsilon \eta}\left(\lambda^{2}, l^{2}\right) .
$$

The following direct products of three-manifolds can be endowed with $\varepsilon$-contact supergravity solutions as indicated in Theorem 5.4 for the values of the parameters specified below:

\footnotetext{
${ }^{3}$ Another solution fitting the case $l=0$ is the non-supersymmetric embedding of the Reissner-Nordström black hole presented in [19].
} 


\begin{tabular}{|c|c|c|c|c|}
\hline \multicolumn{2}{|c|}{ Lorentzian factor } & \multicolumn{2}{|c|}{ Riemannian factor } & \multicolumn{1}{c}{$\left(\lambda^{2}, l^{2}\right)$} \\
\hline$N$ & Sasakian & $X$ & Sasakian & $\lambda^{2}=1+l^{2}, l^{2} \geq 0$ \\
\hline \hline$\widetilde{\mathrm{Sl}}(2, \mathbb{R})$ & Yes & $\mathrm{SU}(2)$ & Yes & $\lambda^{2}=1+l^{2}, l^{2} \geq 0$ \\
\hline $\mathfrak{G}_{6}$ & Yes & $\mathrm{SU}(2)$ & Yes & $\lambda^{2}=0, l^{2}=0$ \\
\hline$\widetilde{\mathrm{E}}(1,1)$ & No & $\widetilde{\mathrm{E}}(2)$ & No & $\lambda^{2}=0, l^{2}=0$ \\
\hline$\widetilde{\mathrm{E}}(2)$ & No & $\widetilde{\mathrm{E}}(2)$ & No & \\
\hline
\end{tabular}

6.3. Null case: $\varepsilon_{N}=0$. Let:

$$
\left(N, \chi, \alpha_{N}, 0\right) \in \operatorname{PCont}_{L}^{\varepsilon \eta}\left(\lambda^{2}, l^{2}, 0\right), \quad\left(X, g, \alpha_{X}\right) \in \operatorname{PCont}_{R}^{\varepsilon \eta}\left(\lambda^{2}, 0\right) .
$$

The product of the following Lorentzian and Riemannian three-manifolds carry $\varepsilon$-contact supergravity solutions as described in Theorem 5.4 for the values of the parameters indicated below:

\begin{tabular}{|c|c|c|c|c|}
\hline \multicolumn{2}{|c|}{ Lorentzian factor } & \multicolumn{2}{|c|}{ Riemannian factor } & \multicolumn{1}{c}{$\left(\lambda^{2}, l^{2}\right)$} \\
\hline$N$ & Sasakian & $X$ & Sasakian & $\lambda^{2}=1, l^{2} \geq 0$ \\
\hline \hline$\widetilde{\mathrm{Sl}}(2, \mathbb{R})$ & Yes & $\mathrm{SU}(2)$ & Yes & $\lambda^{2}=1, l^{2}=0$ \\
\hline $\mathfrak{G}_{6}$ & Yes & $\mathrm{SU}(2)$ & Yes & $\lambda^{2}=1, l^{2}=0$ \\
\hline $\mathfrak{G}_{6}$ & No & $\mathrm{SU}(2)$ & Yes & $\lambda^{2}=0, l^{2} \geq 0$ \\
\hline$\widetilde{\mathrm{E}}(1,1)$ & No & $\widetilde{\mathrm{E}}(2)$ & No & $\lambda^{2}$ \\
\hline
\end{tabular}

Remark 6.1. Note that both Sasakian and non-Sasakian $\varepsilon \eta$-Einstein null-contact structures on $\mathfrak{G}_{6}$ can be combined with Riemannian Sasakian structures on $\mathrm{SU}(2)$ to yield $\varepsilon$-contact supergravity solutions. This is possible due to the fact that the $\varepsilon \eta$-Einstein condition imposes in this case a quadratic constraint on the structure constants. Solutions to this quadratic constraint produce either a Sasakian or a non-Sasakian null contact structure, depending on the particular solution chosen.

\section{Appendix A. Simply COnNeCted three-Dimensional Lorentzian Lie groups}

For the benefit of the reader, we summarize in the following the classification of all threedimensional Lorentzian simply connected Lie groups. The table below is extracted from [17, Theorem 4.1]. In the table below $\widetilde{\mathrm{Sl}}(2, \mathbb{R})$ denotes the universal cover of $\mathrm{Sl}(2, \mathbb{R}), \widetilde{\mathrm{E}}(2)$ denotes the universal cover of the group of rigid motions of the Euclidean plane, whereas $\widetilde{\mathrm{E}}(1,1)$ denotes the universal cover of the group of rigid motions of the Minkowski plane and $\mathrm{H}_{3}$ denotes the threedimensional Heisenberg group.

Theorem A.1. [17, 24, 49] Let (G, g) be a three-dimensional connected, simply connected, Lorentzian Lie group $\mathrm{G}$ with left-invariant metric $\mathrm{g}$. Then, precisely, one of the following cases occurs:

- $\mathrm{G}$ is unimodular and there exists an orthonormal frame $\left\{e_{0}, e_{1}, e_{2}\right\}$, with $e_{0}$ time-like, such that the Lie algebra of $\mathrm{G}$ is one of the following: 
(1) $\mathfrak{g}_{1}$ :

$$
\left[e_{1}, e_{2}\right]=a e_{1}-b e_{0}, \quad\left[e_{1}, e_{0}\right]=-a e_{1}-b e_{2}, \quad\left[e_{2}, e_{0}\right]=b e_{1}+a e_{2}+a e_{0}, \quad a \neq 0
$$

In this case $\mathrm{G} \simeq \widetilde{\mathrm{Sl}}(2, \mathbb{R})$ if $b \neq 0$, while $\mathrm{G} \simeq \widetilde{\mathrm{E}}(1,1)$ if $b=0$.

(2) $\mathfrak{g}_{2}$ :

$$
\left[e_{1}, e_{2}\right]=-c e_{2}-b e_{0}, \quad\left[e_{1}, e_{0}\right]=-b e_{2}+c e_{0}, \quad\left[e_{2}, e_{0}\right]=a e_{1}, \quad c \neq 0
$$

In this case $\mathrm{G} \simeq \widetilde{\mathrm{Sl}}(2, \mathbb{R})$ if $a \neq 0$, while $\mathrm{G} \simeq \widetilde{\mathrm{E}}(1,1)$ if $a=0$.

(3) $\mathfrak{g}_{3}$ :

$$
\left[e_{1}, e_{2}\right]=-c e_{0}, \quad\left[e_{1}, e_{0}\right]=-b e_{2}, \quad\left[e_{2}, e_{0}\right]=a e_{1},
$$

In this case the isomorphism type of $\mathrm{G}$ is listed in the following table.

\begin{tabular}{|c||c|c|c|}
\hline \multicolumn{3}{|c|}{ Simply-connected unimodular groups with Lie algebra $\mathfrak{g}_{3}$} \\
\hline Lie group $\mathrm{G}$ & $a$ & $b$ & $c$ \\
\hline$\widetilde{\mathrm{S}}(2, \mathbb{R})$ & + & + & + \\
$\widetilde{\mathrm{S}}(2, \mathbb{R})$ & + & - & - \\
$\mathrm{SU}(2)$ & + & + & - \\
$\widetilde{\mathrm{E}}(2)$ & + & + & - \\
$\widetilde{\mathrm{E}}(2)$ & + & 0 & 0 \\
$\widetilde{\mathrm{E}}(1,1)$ & + & - & + \\
$\widetilde{\mathrm{E}}(1,1)$ & + & 0 & 0 \\
$\mathrm{H}_{3}$ & + & 0 & - \\
$\mathrm{H}_{3}$ & 0 & 0 & 0 \\
$\mathbb{R} \oplus \mathbb{R} \oplus \mathbb{R}$ & 0 & 0 & \\
\hline
\end{tabular}

(4) $\mathfrak{g}_{4}:$

$$
\left[e_{1}, e_{2}\right]=-e_{2}+(2 \mu-b) e_{0}, \quad\left[e_{1}, e_{0}\right]=-b e_{2}+e_{0}, \quad\left[e_{2}, e_{0}\right]=a e_{1}, \quad \mu \in \mathbb{Z}_{2}
$$

In this case the isomorphism type of $\mathrm{G}$ is listed in the following tables.

\begin{tabular}{|c||c|c|}
\hline \multicolumn{2}{|c|}{ Simply-connected unimodular groups with Lie algebra $\mathfrak{g}_{4}$ and $\mu=1$} \\
\hline Lie group $\mathrm{G}$ & $a$ & $b$ \\
\hline$\widetilde{\mathrm{Sl}}(2, \mathbb{R})$ & $\neq 0$ & $\neq 1$ \\
$\widetilde{\mathrm{E}}(1,1)$ & 0 & $\neq 1$ \\
$\widetilde{\mathrm{E}}(1,1)$ & $<0$ & 1 \\
$\widetilde{\mathrm{E}}(2)$ & $>0$ & 1 \\
$\mathrm{H}_{3}$ & 0 & 1 \\
\hline
\end{tabular}




\begin{tabular}{|c||c|c|}
\hline \multicolumn{2}{|c|}{ Simply-connected unimodular groups with Lie algebra $\mathfrak{g}_{4}$ and $\mu=-1$} \\
\hline Lie group $\mathrm{G}$ & $a$ & $b$ \\
\hline$\widetilde{\mathrm{S}}(2, \mathbb{R})$ & $\neq 0$ & $\neq-1$ \\
$\widetilde{\mathrm{E}}(1,1)$ & 0 & $\neq-1$ \\
$\widetilde{\mathrm{E}}(1,1)$ & $>0$ & -1 \\
$\widetilde{\mathrm{E}}(2)$ & $<0$ & -1 \\
$\mathrm{H}_{3}$ & 0 & -1 \\
\hline
\end{tabular}

- $\mathrm{G}$ is non-unimodular and there exists an orthonormal frame $\left\{e_{0}, e_{1}, e_{2}\right\}$, with $e_{0}$ time-like, such that the Lie algebra of $\mathrm{G}$ is one of the following:

(1) $\mathfrak{g}_{5}$ :

$$
\begin{gathered}
{\left[e_{1}, e_{2}\right]=0, \quad\left[e_{1}, e_{0}\right]=a e_{1}+b e_{2}, \quad\left[e_{2}, e_{0}\right]=c e_{1}+d e_{2},} \\
a+d \neq 0, \quad a c+b d=0 .
\end{gathered}
$$

We denote the unique connected and simply connected Lie group with Lie algebra $\mathfrak{g}_{5}$ as $\mathfrak{G}_{5}$. (2) $\mathfrak{g}_{6}$ :

$$
\begin{gathered}
{\left[e_{1}, e_{2}\right]=a e_{2}+b e_{0}, \quad\left[e_{1}, e_{0}\right]=c e_{2}+d e_{0}, \quad\left[e_{2}, e_{0}\right]=0,} \\
a+d \neq 0, \quad a c-b d=0 .
\end{gathered}
$$

We denote the unique connected and simply connected Lie group with Lie algebra $\mathfrak{g}_{6}$ as $\mathfrak{G}_{6}$. (3) $\mathfrak{g}_{7}$ :

$$
\begin{gathered}
{\left[e_{1}, e_{2}\right]=-a e_{1}-b e_{2}-b e_{0}, \quad\left[e_{1}, e_{0}\right]=a e_{1}+b e_{2}+b e_{0}, \quad\left[e_{2}, e_{0}\right]=c e_{1}+d e_{2}+d e_{0},} \\
a+d \neq 0, \quad a c=0 .
\end{gathered}
$$

We denote the unique connected and simply connected Lie group with Lie algebra $\mathfrak{g}_{7}$ as $\mathfrak{G}_{7}$.

Non-unimodular Lie algebras can be interpreted as deformations of the unimodular ones, with deformation parameter given by:

$$
\beta \stackrel{\text { def. }}{=} a+d .
$$

This is due to the fact that in the limit $\beta \rightarrow 0$ all the previous non-unimodular Lie algebras reduce to unimodular Lie algebras [44]. Consider as an example the Lie algebra $\mathfrak{g}_{6}$ in the limit $\beta \rightarrow 0$. In such limit the Lie brackets of $\mathfrak{g}_{6}$ reduce to:

$$
\begin{gathered}
{\left[e_{1}, e_{2}\right]=a e_{2}+b e_{0}, \quad\left[e_{1}, e_{0}\right]=c e_{2}-a e_{0}, \quad\left[e_{2}, e_{0}\right]=0,} \\
a(c+b)=0 .
\end{gathered}
$$

The specific unimodular algebras occurring in the limit $\beta \rightarrow 0$ of $\mathfrak{g}_{6}$ can be easily identified in some particular cases:

- If $a=0$, we obtain:

$$
\left[e_{1}, e_{2}\right]=b e_{0}, \quad\left[e_{1}, e_{0}\right]=c e_{2}, \quad\left[e_{2}, e_{0}\right]=0 .
$$

Comparing with the classification of unimodular Lie algebras, we conclude that the case $c b>0$ corresponds to the Lie algebra of $\widetilde{\mathrm{E}}(1,1)$, the case $c b<0$ corresponds to the Lie algebra of $\widetilde{\mathrm{E}}(2)$, the case $c \neq 0, b=0$ or $c=0, b \neq 0$ corresponds to the Lie algebra of $\mathrm{H}_{3}$ and the case $c=b=0$ corresponds to $\mathbb{R}^{3}$. 
- If $a \neq 0$ then we must have $c=-b$. If in addition we set $c=0$, the brackets take the form:

$$
\left[e_{1}, e_{2}\right]=a e_{2}, \quad\left[e_{1}, e_{0}\right]=-a e_{0}, \quad\left[e_{2}, e_{0}\right]=0
$$

Comparing with the classification of unimodular Lie algebras, we conclude that this algebra is isomorphic to $\widetilde{\mathrm{E}}(1,1)$.

- Also, if $a=b=1$ and $c=-b=-1$ the brackets read:

$$
\left[e_{1}, e_{2}\right]=e_{0}+e_{2}, \quad\left[e_{1}, e_{0}\right]=-e_{0}-e_{2}, \quad\left[e_{2}, e_{0}\right]=0 .
$$

which defines a Lie algebra isomorphic to $\mathrm{H}_{3}$.

Therefore, the non-unimodular Lie group $\mathfrak{G}_{6}$ can be understood as a deformation of various unimodular groups such as $\widetilde{\mathrm{E}}(1,1), \widetilde{\mathrm{E}}(2)$ or $\mathrm{H}_{3}$. Similar remarks hold for $\mathfrak{G}_{5}$ and $\mathfrak{G}_{7}$.

Appendix B. Curvature of left-InVariant metrics on Lorentzian Lie groups

Let $(\mathrm{G}, g)$ be a three-dimensional Lorentzian Lie group with Lie algebra $\mathfrak{g}$. Fix a global leftinvariant frame $\left\{e_{0}, e_{1}, e_{2}\right\}$ on $\mathrm{G}$. We have:

$$
\left[e_{0}, e_{1}\right]=a e_{0}+b e_{1}+c e_{2}, \quad\left[e_{1}, e_{2}\right]=d e_{0}+f e_{1}+h e_{2} \quad\left[e_{0}, e_{2}\right]=g e_{0}+j e_{1}+k e_{2},
$$

where $a, b, c, d, f, h, g, j, k \in \mathbb{R}$ such that the Jacobi identity is satisfied. In this case, imposing $\left[\left[e_{0}, e_{1}\right], e_{2}\right]+\left[\left[e_{2}, e_{0}\right], e_{1}\right]+\left[\left[e_{1}, e_{2}\right], e_{0}\right]=0$ yields the constraints

$$
b d+k d-f a-h g=0, \quad j a-g b+k f-h j=0, \quad a k+h b-g c-f c=0 .
$$

Using Koszul formula, we compute the following covariant derivatives:

$$
\begin{array}{ccc}
\nabla_{e_{0}} e_{0}=a e_{1}+g e_{2}, & \nabla_{e_{0}} e_{1}=a e_{0}+\frac{(c-j+d)}{2} e_{2}, & \nabla_{e_{0}} e_{2}=g e_{0}+\frac{(j-c-d)}{2} e_{1}, \\
\nabla_{e_{1}} e_{0}=-b e_{1}+\frac{(-c+d-j)}{2} e_{2}, & \nabla_{e_{1}} e_{1}=-b e_{0}-f e_{2}, & \nabla_{e_{1}} e_{2}=\frac{(d-c-j)}{2} e_{0}+f e_{1}, \\
\nabla_{e_{2}} e_{0}=-\frac{(j+d+c)}{2} e_{1}-k e_{2}, & \nabla_{e_{2}} e_{1}=-\frac{(j+d+c)}{2} e_{0}-h e_{2}, & \nabla_{e_{2}} e_{2}=-k e_{0}+h e_{1} .
\end{array}
$$


Using the previous covariant derivatives we compute the Riemann curvature tensor (with the convention $\left.\mathrm{R}^{g}(u, v) w=\nabla_{u} \nabla_{v} w-\nabla_{v} \nabla_{u} w-\nabla_{[u, v]} w\right)$ :

$$
\begin{aligned}
& \mathrm{R}^{g}\left(e_{0}, e_{1}\right) e_{0}=\left[-a^{2}+b^{2}-g f+\frac{1}{4}(-c+d-j)(j-c-d)+\frac{c}{2}(j+d+c)\right] e_{1} \\
& +[-b d+a f-a g+c k+b j] e_{2}, \\
& \mathrm{R}^{g}\left(e_{0}, e_{1}\right) e_{1}=\left[-a^{2}+b^{2}-g f+\frac{1}{4}(-c+d-j)(j-c-d)+\frac{c}{2}(j+d+c)\right] e_{0} \\
& +[-b g+b f-a d+h c+a j] e_{2}, \\
& \mathrm{R}^{g}\left(e_{0}, e_{1}\right) e_{2}=[f a-a g+b j-b d+c k] e_{0}+[a d-a j+g b-b f-c h] e_{1}, \\
& \mathrm{R}^{g}\left(e_{1}, e_{2}\right) e_{0}=[-k f+f b-d a+h c+h j] e_{1}+[-b h+h k-d g+f j+f c] e_{2}, \\
& \mathrm{R}^{g}\left(e_{1}, e_{2}\right) e_{1}=[h c+h j-f k-d a+f b] e_{0} \\
& +\left[-b k+f^{2}+h^{2}-\frac{d}{2}(c-j+d)-\frac{1}{4}(d+j+c)(-c+d-j)\right] e_{2}, \\
& \mathrm{R}^{g}\left(e_{1}, e_{2}\right) e_{2}=[-b h+f j+f c-d g+h k] e_{0} \\
& +\left[\left[b k-f^{2}-h^{2}+\frac{d}{2}(c-j+d)+\frac{1}{4}(d+j+c)(-c+d-j)\right] e_{1},\right. \\
& \mathrm{R}^{g}\left(e_{0}, e_{2}\right) e_{0}=[k c+k d-g h-g a+j b] e_{1} \\
& +\left[a h-g^{2}+k^{2}-\frac{j}{2}(-c+d-j)-\frac{1}{4}(j+d+c)(c-j+d)\right] e_{2}, \\
& \mathrm{R}^{g}\left(e_{0}, e_{2}\right) e_{1}=[-h g-g a+j b+k c+k d] e_{0}+[a k+j f+k h-g c-g d] e_{2}, \\
& \mathrm{R}^{g}\left(e_{0}, e_{2}\right) e_{2}=\left[\frac{1}{4}(j-c-d)(d+j+c)-g^{2}+k^{2}+\frac{j}{2}(-d+c+j)+a h\right] e_{0} \\
& +[-k a+g d+g c-f j-k h] e_{1},
\end{aligned}
$$

From here, we obtain the Ricci curvature tensor $\operatorname{Ric}^{g}$ defined as $\operatorname{Ric}^{g}(u, v)=\operatorname{Tr}\left(w \rightarrow \mathrm{R}^{g}(w, u) v\right)$.

$$
\begin{aligned}
& \operatorname{Ric}^{g}\left(e_{0}, e_{0}\right)=a^{2}-b^{2}+g f+g^{2}-k^{2}-a h+\frac{d^{2}}{2}-c j-\frac{c^{2}}{2}-\frac{j^{2}}{2}, \\
& \operatorname{Ric}^{g}\left(e_{0}, e_{1}\right)=b h-f j-f c+d g-h k \\
& \operatorname{Ric}^{g}\left(e_{0}, e_{2}\right)=h c+h j-f k-d a+f b \\
& \operatorname{Ric}^{g}\left(e_{1}, e_{1}\right)=-a^{2}+b^{2}-f^{2}-h^{2}-f g+b k-\frac{j^{2}}{2}+d c+\frac{c^{2}}{2}+\frac{d^{2}}{2}, \\
& \operatorname{Ric}^{g}\left(e_{1}, e_{2}\right)=f a-a g+b j-b d+c k \\
& \operatorname{Ric}^{g}\left(e_{2}, e_{2}\right)=-g^{2}+k^{2}+a h+k b-f^{2}-h^{2}+\frac{j^{2}}{2}-j d+\frac{d^{2}}{2}-\frac{c^{2}}{2} .
\end{aligned}
$$

Finally, the scalar curvature $\mathrm{Scal}^{g}$ reads

$$
\mathrm{Scal}^{g}=-2 a^{2}+2 b^{2}-2 g f-2 g^{2}+2 k^{2}+2 a h+\frac{d^{2}}{2}+c j+\frac{c^{2}}{2}+\frac{j^{2}}{2}-2 f^{2}-2 h^{2}+2 b k+d c-j d .
$$




\section{REFERENCES}

[1] M. Akyol and G. Papadopoulos, Spinorial geometry and Killing spinor equations of 6-D supergravity, Class. Quant. Grav. 28 (2011) 105001.

[2] M. Akyol and G. Papadopoulos, Topology and geometry of 6-dimensional $(1,0)$ supergravity black hole horizons, Class. Quant. Grav. 29 (2012) 055002.

[3] D. V. Alekseevski, V. Cortés, A. S. Galaev and T. Leistner Cones over pseudo-Riemannian manifolds and their holonomy, J. Reine Angew. Math. 635 (2009), 23 - 69.

[4] L. Andersson, V. Moncrief and A. J. Tromba, On the global evolution problem in $(2+1)$ gravity, J. Geom. Phys. 23 (1997) 191.

[5] H. Baum, T. Leistner, A. Lischewski, Cauchy problems for Lorentzian manifolds with special holonomy, Differential Geometry and its Applications, Volume 45, (2016), 43 - 66.

[6] I. Bena and N. P. Warner, Black holes, black rings and their microstates, Lect. Notes Phys. $755(2008) 1$.

[7] I. Bena, S. Giusto, M. Shigemori and N. P. Warner, Supersymmetric Solutions in Six Dimensions: A Linear Structure, JHEP 1203 (2012) 084.

[8] A. N. Bernal and M. Sánchez, On Smooth Cauchy hypersurfaces and Geroch's splitting theorem, Commun. Math. Phys. 243 (2003) 461.

[9] D. E. Blair, Riemannian Geometry of Contact and Symplectic Manifolds, Birkhäuser, Progress in Mathematics (2010).

[10] D. E. Blair, T. Koufogiorgos and R. Sharma A classification of three-dimensional contact metric manifolds with $Q \varphi=\varphi Q$, Kodai. Math J. 13 (1990), 391 - 401.

[11] D. E. Blair, T. Koufogiorgos and B. J. Papantoniou Contact metric manifolds satisfying a nullity condition, Israel J. Math. (1995) 91: 189.

[12] A. V. Bolsinov, A. Yu. Konyaev, V. S. Matveev, Nijenhuis Geometry, arXiv:1903.04603.

[13] Charles Boubel, An integrability condition for fields of nilpotent endomorphisms, arXiv: 1003.0979.

[14] J.-L. Brylinski, Loop Spaces, Characteristic Classes and Geometric Quantization, Modern Birkhäuser Classics, 1993.

[15] G. Calvaruso, Contact Lorentzian manifolds, Birkhäuser, Differential Geometry and its Applications Volume 29, Supplement 1, August 2011.

[16] G. Calvaruso, Homogeneous paracontact metric three-manifolds, Illinois Journal of Mathematics Volume 55, Number 2, Summer 2011, Pages 697 - 718.

[17] G. Calvaruso, Homogeneous structures on three-dimensional Lorentzian manifolds, Journal of Geometry and Physics 57 (2007) 1279 - 1291.

[18] G. Calvaruso, Geometry of H-paracontact metric manifolds, Publicationes mathematicae 86 $(3-4)$.

[19] P. A. Cano, S. Chimento, R. Linares, T. Ortín and P. F. Ramírez, $\alpha^{\prime}$ corrections of ReissnerNordström black holes, arXiv:1910.14324 [hep-th].

[20] P. A. Cano and T. Ortín, The structure of all the supersymmetric solutions of ungauged $\mathcal{N}=(1,0), d=6$ supergravity, Class. Quant. Grav. 36 (2019) no.12, 125007.

[21] S. Cecotti, Supersymmetric Field Theories: Geometric Structures and Dualities, Cambridge University Press; (2015).

[22] A. Chamseddine, J. M. Figueroa-O'Farrill and W. Sabra, Supergravity vacua and Lorentzian Lie groups, arXiv:0306278.

[23] Y. Choquet-Bruhat, The Cauchy problem in extended supergravity, $N=1, d=11$, Comm. Math. Phys. Volume 97, Number 4 (1985), 541 - 552. 
[24] L. A. Cordero and P. E. Parker, Left-invariant Lorentzian metrics on 3-dimensional Lie groups, Rend. Mat. Appl. (7) 17 (1997) 129 - 155.

[25] P. de Medeiros, J. Figueroa-O'Farrill and A. Santi, Killing superalgebras for Lorentzian sixmanifolds, J. Geom. Phys. 132 (2018) 13.

[26] K. L. Duggal, Space-time manifolds and contact structures, Internat. J. Math. \& Math. Sci. VOL. 13 NO. 3 (1990) $545-554$.

[27] S. Dumitrescu and A. Zeghib, Géométries Lorentziennes de dimension 3 : classification et complétude, Birkhäuser, Progress in Mathematics (2010).

[28] J. Figueroa-O'Farrill and N. Hustler, The homogeneity theorem for supergravity backgrounds II: the six-dimensional theories, JHEP 1404 (2014) 131.

[29] Charles Frances, Lorentz Dynamics on Closed 3-Manifolds, arXiv:1804.08695.

[30] D. Z. Freedman, A. Van Proeyen, supergravity, Cambridge Monographs on Mathematical Physics, 2012.

[31] A. A. García-Díaz, Exact solutions in three-dimensional gravity, Cambridge University Press (2017).

[32] M. Garcia-Fernandez and C. S. Shahbazi, Self-dual generalized metrics for pure $\mathcal{N}=1$ sixdimensional Supergravity, arXiv:1505.03088 [hep-th].

[33] H. Geiges, Normal contact structures on 3-manifolds, Tohoku Math. J. (2) Volume 49, Number 3 (1997), 415 - 422.

[34] J. B. Gutowski, D. Martelli and H. S. Reall, All Supersymmetric solutions of minimal supergravity in six- dimensions, Class. Quant. Grav. 20 (2003) 5049.

[35] H. het Lam and S. Vandoren, BPS solutions of six-dimensional $(1,0)$ supergravity coupled to tensor multiplets, JHEP 1806 (2018) 021.

[36] E. T. Kobayashi, A remark on the Nijenhius tensor, Pacific J. Math. 12 (1961), 963 - 977.

[37] S. Kobayashi, K. Nomizu, Foundations of Differential Geometry Volume I, John Wiley and Sons (1963)

[38] T. Koufogiorgos, On a Class of Contact Riemannian 3-Manifolds, Results. Math. (1995) 27: 51.

[39] J. Lehmann - Lejeune, Integrabilaté des G-structures définies par une 1-forme 0-déformable à valeurs dans le fibré tangent, Ann. Inst. Fourier 16 (1966), 329 - 387.

[40] H. Lin, O. Lunin and J. Maldacena, Bubbling AdS space and 1/2 BPS geometries, JHEP 0410, 025 (2004).

[41] J. T. Liu and D. Vaman, Bubbling 1/2 BPS solutions of minimal six-dimensional supergravity, Phys. Lett. B 642 (2006) 411.

[42] D. Martelli and J. F. Morales, Bubbling AdS(3), JHEP 0502 (2005) 048.

[43] S. D. Mathur, The Information paradox: A Pedagogical introduction, Class. Quant. Grav. 26 (2009) 224001.

[44] J. Milnor, Curvatures of left invariant metrics on Lie groups, Advances in Mathematics 21, 293 - 329 (1976).

[45] V. Moncrief, Reduction of the Einstein equations in (2+1)-dimensions to a Hamiltonian system over Teichmuller space, J. Math. Phys. 30 (1989) 2907.

[46] H. Nishino and E. Sezgin, Matter and Gauge Couplings of N=2 Supergravity in SixDimensions, Phys. Lett. 144B (1984) 187.

[47] H. Nishino and E. Sezgin, The Complete $N=2, d=6$ Supergravity With Matter and YangMills Couplings, Nucl. Phys. B 278 (1986) 353.

[48] T. Ortín, Gravity and Strings, Cambridge Monographs on Mathematical Physics, 2nd edition, 
2015.

[49] S. Rahmani, Métriques de Lorentz sur les groupes de Lie unimodulaires, de dimension trois, J. Geom. Phys. 9 (1992), 295 - 302.

[50] G. Thompson, The integrability of a field of endomorphisms, Mathematica Bohemica, Vol. 127 (2002), No. 4, 605-611.

[51] F. Tricerri, L. Vanhecke, Homogeneous structures on Riemannian manifolds, London Math. Soc. Lecture Notes Series, 83, Cambridge University Press, London, 1983.

[52] F. J. Turiel, Intégrabilité d'un tenseur de type (1,1) et structure symplectique du fibré cotangent, C.R. Acad. Sci., Paris, Sér.I 301 (1985), 923 - 925.

[53] S. Zamkoyov, Canonical connections on paracontact manifolds, Ann. Glob. Anal. Geom. 36 (2009), 37-60. MR 2520029

[54] S. Zamkoyov, A classification of 3-dimensional paracontact metric manifolds with $Q \varphi=\varphi Q$, arXiv:1910.04593 [math.DG].

Instituto de Física Teórica, CSiC, Madrid, Reino de España.

Email address: angel.murcia@csic.es

Fachbereich Mathematik, Hamburg Universität, Deutschland.

Email address: carlos.shahbazi@uni-hamburg.de 